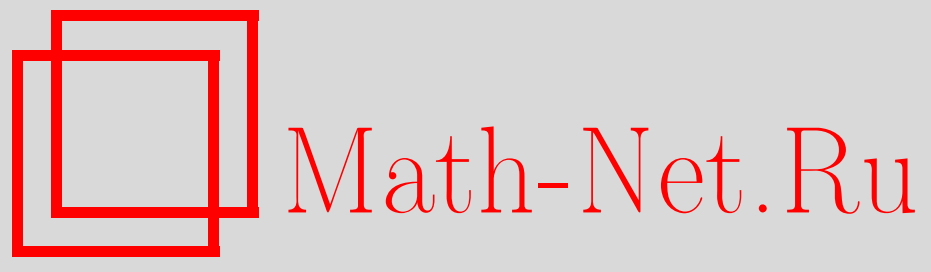

А. В. Пухликов, Бирационально жесткие многообразия. I. Многообразия Фано, УМH, 2007, том 62, выпуск 5, 15106

DOI: https://doi.org/10.4213/rm8505

Использование Общероссийского математического портала Math-Net.Ru подразумевает, что вы прочитали и согласны с пользовательским соглашением http://www . mathnet.ru/rus/agreement

Параметры загрузки:

IP: 3.82 .47 .9

26 апреля 2023 г., 14:52:00

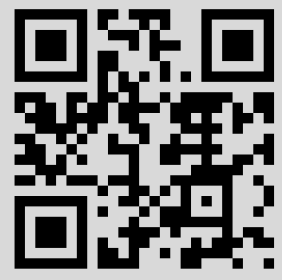




\title{
Бирационально жесткие многообразия. I. Многообразия Фано
}

\begin{abstract}
А. В. Пухликов
Теория бирациональной жесткости рационально связных многообразий обобщает классическую проблему рациональности. В статье дается обзор современного состояния этой теории и прослеживается ее история от теоремы Нётера и проблемы Люрота до последних результатов о бирациональной сверхжесткости многомерных многообразий Фано. Рассмотрены основные звенья метода максимальных особенностей.

Библиография: 86 названий.
\end{abstract}

\section{СОДЕРЖАНИЕ}

Введение . . .......................................... 16

Глава 1. Проблема рациональности и ее обобщения ................. 17

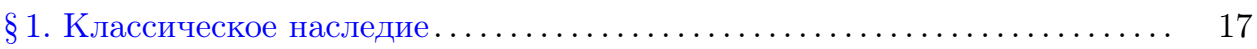

1.1. Проблема рациональности. Примеры .................... 17

1.2. Бирациональная геометрия ....................... 20

1.3. Две классические теоремы .......................... 23

$\S 2$. Первые шаги современной бирациональной геометрии ........... 28

2.1. Работы Фано....................................... 28

2.2. Решение проблемы Люрота ......................... 31

2.3. Теорема Саркисова о расслоениях на коники .............. 34

$\S 3$. Рационально связные многообразия ....................... 38

3.1. Теорема Грабера-Харриса-Старра.................... 38

3.2. Рационально связные расслоения и расслоения Фано ......... 41

3.3. Метод максимальных особенностей .................... 44

Глава 2. Метод максимальных особенностей...................... 47

$\S 1$. Обрыв канонического присоединения ..................... 47

1.1. Порог канонического присоединения и бирациональная жест-

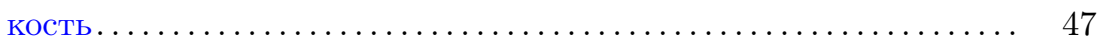

1.2. Особенности линейных систем ....................... 51

1.3. Общая схема метода ............................ 56

(C) А.В. Пухликов, 2007 
$\S 2$ Исключение максимальных особенностей..................... 59

2.1. Максимальные подмногообразия.................... 59

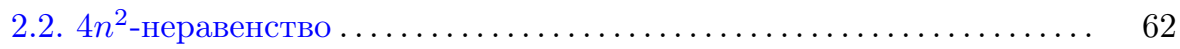

2.3. Второе доказательство: принцип связности .............. 65

$\S 3$. Откручивание максимальных особенностей ................. 69

3.1. Откручивающие бирациональные автоморфизмы ........... 69

3.2. Полное пересечение квадрики и кубики: образующие ......... 71

3.3. Полное пересечение квадрики и кубики: соотношения......... 74

Глава 3. Гиперкасательные дивизоры и линейные системы ........... 78

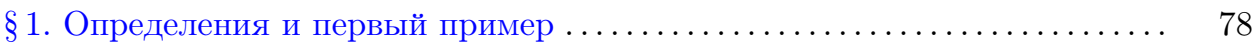

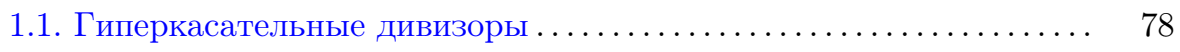

1.2. Гиперкасательные линейные системы .................. 82

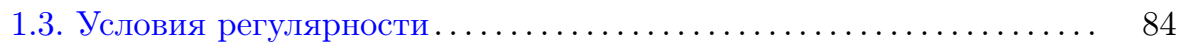

$\S 2$. Полные пересечения Фано . . . . . . . . . . . . . . . . . . . 86

2.1. Теорема о бирациональной сверхжесткости.............. 86

2.2. Построение гиперкасательных дивизоров ................ 88

2.3. Циклические накрытия . . . . . . . . . . . . . . . . . . . . . 93

$\S 3$. Регулярные многообразия Фано ............................ 94

3.1. Метод оценки коразмерности . ...................... 94

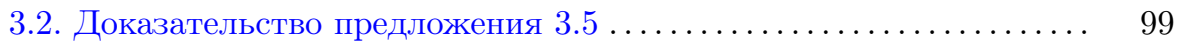

3.3. Заключительные замечания......................... 101

Список литературы .................................. 101

\section{Введение}

Цель настоящей статьи - дать обзор современного состояния теории бирациональной жесткости многообразий Фано. Этой проблематике уже посвящены два недавно опубликованных обзора [1], [2], так что появление еще одной работы нуждается в пояснении. Теория бирациональной жесткости, ведущая свою историю с пионерской статьи В.А. Исковских и Ю. И. Манина [3], является одной из главных компонент современной бирациональной геометрии. Она возникла независимо от программы минимальных моделей и развивалась параллельно ей, в некоторых аспектах с ней пересекаясь. Важность и сила результатов теории бирациональной жесткости делают ее достойной внимания широких кругов алгебраических геометров и всех, кто так или иначе интересуется алгебраической геометрией. Однако обзоры [1], [2] рассчитаны на специалистов, работающих в бирациональной геометрии (особенно обзор [2], перегруженный терминологией программы минимальных моделей); кроме того, в этих обзорах мало внимания уделяется основным результатам и методам теории бирациональной жесткости в произвольной размерности.

Настоящая статья представляет собой одновременно и обзор теории бирациональной жесткости, и введение в эту теорию. Первая (вводная) глава рассчитана на широкую математическую аудиторию. Последующий материал предполагает знакомство с основами алгебраической геометрии. Данный обзор посвящен бирациональной геометрии многообразий Фано; геометрии расслоений Фано автор предполагает посвятить отдельную обзорную статью. 
Принцип нумерации утверждений, определений, замечаний и т. п. в работе следующий: теорема $a . b$ относится к главе $a$ и является в этой главе $b$-й. При ссылке на параграф (§a) или пункт (п. a.b), если номер главы не указан, то подразумевается текущая глава.

Автор благодарит рецензента за ряд полезных замечаний.

\section{Глава 1. Проблема рациональности и ее обобщения}

\section{§1. Классическое наследие}

1.1. Проблема рациональности. Примеры. Проблема рациональности является одной из самых старых проблем алгебраической геометрии; одной из тех проблем, с которых алгебраическая геометрия началась. В первом приближении алгебраическую геометрию можно понимать как область математики, изучающую геометрию алгебраических множеств, т. е. множеств решений систем алгебраических уравнений

$$
\left\{\begin{array}{l}
f_{1}\left(x_{1}, \ldots, x_{n}\right)=0 \\
\ldots \ldots \ldots \ldots \ldots \ldots \\
f_{m}\left(x_{1}, \ldots, x_{n}\right)=0
\end{array}\right.
$$

где $f_{i}\left(x_{*}\right)$ - многочлены. Если все многочлены $f_{i}$ линейны, то (при условии, что система совместна) множество переменных $x_{1}, \ldots, x_{n}$ можно разбить на два непересекающихся подмножества: главных переменных $x_{i}, i \in I$, и свободных переменных $x_{i}, i \in J$, где $I \vee J=\{1, \ldots, n\}$. Общее решение системы (1) при этом выглядит так: главные переменные суть линейные функции от свободных переменных, причем последние могут принимать произвольные значения. Иначе говоря, множество решений системы (1) можно параметризовать, а в качестве естественных параметров взять множество свободных переменных.

Принимая за модель описанную идеальную картину линейного мира, можно задать наивный вопрос: возможно ли параметризовать множество решений произвольной системы (1) нелинейных полиномиальных уравнений? Это и есть проблема рациональности. Осознание того, что для общей системы (1) ответ на этот вопрос является отрицательным, было, по-видимому, одним из ключевых моментов в начальный период алгебраической геометрии. Проблема рациональности - это проблема явного решения системы алгебраических уравнений. Ее отрицательное решение означает, что явного решения не существует.

Однако здесь необходимо сделать две оговорки. Во-первых, даже в достаточно простых случаях, когда система уравнений (1) имеет параметризуемое множество решений, почти никогда нельзя взять в качестве параметров набор координатных функций $x_{i}, i \in J$, по аналогии с линейным случаем. И во-вторых, почти никогда нельзя параметризовать множество решений целиком: самое большее, на что можно надеяться, - это параметризация "почти всех" решений, за исключением собственного подмножества меньшей размерности. Наиболее близкая общематематическая аппроксимация данного замечания - это параметризация открытого плотного подмножества множества решений рациональными функциями от координат $x_{*}$. 
И еще одна оговорка. Проблема рациональности имеет очень важный арифметический аспект, однако в данном обзоре мы ограничимся ее рассмотрением в контексте комплексной алгебраической геометрии. Все объекты определены над полем комплексных чисел $\mathbb{C}$.

ОПРЕДЕЛЕНИЕ 1.1. Комплексное алгебраическое многообразие $V$ называется рациональным, если выполнено любое из следующих эквивалентных условий:

- существует непустое открытое по Зарискому подмножество $U \subset V$, изоморфное открытому алгебраическому подмножеству $U^{+} \subset \mathbb{C}^{n}, n=\operatorname{dim} V$;

- существует непустое открытое по Зарискому подмножество $U \subset V$, допускающее глобальную систему координат;

- существует алгебраическое соответствие $\varphi: V \rightarrow \rightarrow \mathbb{C}^{n}$, взаимно однозначное на открытых плотных подмножествах.

Имеется и чисто алгебраический вариант определения:

- поле рациональных функций $\mathbb{C}(V)$ многообразия $V$ изоморфно полю рациональных функций от независимых переменных, $\mathbb{C}(V) \cong \mathbb{C}\left(t_{1}, \ldots, t_{n}\right)$.

ПримеР 1.1. (i) Проективное пространство $\mathbb{P}^{n}$ с однородными координатами $\left(x_{0}: x_{1}: \ldots: x_{n}\right)$ покрывается стандартными аффинными картами $U_{i}=\left\{x_{i} \neq 0\right\} \cong \mathbb{C}^{n}$ и потому рационально.

(ii) Грассманианы $\operatorname{Gr}(k, n)$ покрываются картами $U_{I}=\left\{\operatorname{det} A_{I} \neq 0\right\} \cong$ $\mathbb{C}^{k(n-k)}$ и потому рациональны (подразумевается реализация $\operatorname{Gr}(k, n)$ как факторпространства $\operatorname{Hom}\left(\mathbb{C}^{k}, \mathbb{C}^{n}\right)$ (отождествленного с пространством $(k \times n)$-матриц) по действию группы Aut $\left.\mathbb{C}^{k}\right)$.

(iii) Торические многообразия содержат алгебраический тор $\left(\mathbb{C}^{*}\right)^{\times n}$ в качестве плотного открытого подмножества, $\mathbb{C}^{*}=\mathbb{C} \backslash\{0\}$, и потому рациональны.

(iv) Квадратичная гиперповерхность $Q \subset \mathbb{P}^{n}$ рациональна: стереографическая проекция из неособой точки $p \in Q$ параметризует квадрику $Q$. В самом деле, пусть $P \cong \mathbb{P}^{n-1}-$ произвольная гиперплоскость, не содержащая точку $p$. Для любой точки $x \in Q \backslash\{p\}$ пусть $L_{x p} \subset \mathbb{P}^{n}-$ прямая, соединяющая $x$ и $p$. Положим $\pi(x)=L_{x p} \cap P$. Тем самым определено отображение $\pi: Q \backslash\{p\} \rightarrow P$. Понятно, что любая точка $q \in P$ имеет в точности один прообраз $\pi^{-1}(q)$, если только прямая $L_{q p}$ не содержится целиком в $Q$.

Пусть $T \subset \mathbb{P}^{n}-$ (единственная) гиперплоскость, касающаяся $Q$ в точке $p$. Легко видеть, что любая прямая $L \ni p$ целиком содержится в $Q$ тогда и только тогда, когда $L \subset T$, и обратно, $Q \cap T$ есть квадратичный конус с вершиной в точке $p$. Следовательно, стереографическая проекция определяет изоморфизм открытых множеств $Q \backslash(Q \cap T)$ и $P \backslash(P \cap T)$.

(v) Менее тривиальный пример - кубические поверхности. Пусть $Q \subset \mathbb{P}^{3}-$ неособая кубическая поверхность. Одним из достижений классической алгебраической геометрии было установление следующего факта (вошедшего, по-видимому, во все современные учебники алгебраической геометрии [4]-[7]): на поверхности $Q$ лежат ровно 27 прямых. Конфигурация этих прямых изучена до мельчайших подробностей, однако для того, чтобы доказать рациональность поверхности $Q$, достаточно существования двух непересекающихся 
прямых $L_{+}, L_{-} \subset Q$. Очевидно, что $\left\langle L_{+}, L_{-}\right\rangle=\mathbb{P}^{3}$ (угловые скобки $\langle\cdot, \cdot\rangle$ обозначают линейную оболочку). Пусть $x_{+} \in L_{+}$и $x_{-} \in L_{-}-$точки общего положения. Прямая $L$, соединяющая $x_{+}$и $x_{-}$, пересекает $Q$ в трех точках, две из которых нам известны: $L \cap Q=\left\{x_{+}, x_{-}, x\right\}$. Сопоставление $\left(x_{+}, x_{-}\right) \mapsto x$ корректно определяет отображение

$$
\gamma:\left(L_{+} \times L_{-}\right) \backslash Y \rightarrow Q
$$

где $Y$ состоит из пар $\left(x_{+}, x_{-}\right)$, для которых прямая $L$ целиком содержится в $Q$. Таких пар конечное число, так что множество $Y$ конечно и $\gamma$ есть отображение $\left(\mathbb{P}^{1} \times \mathbb{P}^{1}\right) \backslash Y \rightarrow Q$. Легко понять, далее, что для точки общего положения $x \in \mathbb{P}^{3}$ существует единственная пара точек $x_{ \pm} \in L_{ \pm}$такая, что $x \in L$. В терминах подходящих однородных координат на $\mathbb{P}^{3}$ сопоставление $\beta: x \mapsto\left(x_{+}, x_{-}\right)$соответствует изоморфизму $\mathbb{C}^{4} \cong \mathbb{C}^{2} \oplus \mathbb{C}^{2}$. Отображение $\beta$ не определено в точках $x \in L_{+} \cup L_{-}$. Очевидно, что $\beta$ и $\gamma$ взаимно обратны, что и доказывает рациональность кубической поверхности $Q$.

Приведенные примеры геометрически элементарны, однако один шаг отделяет последний пример от современной алгебраической геометрии: повысив размерность кубики на единицу, получаем следующий нетривиальный факт.

Теорема 1.1 (Г. Клеменс, Ф. Гриффитс, 1971, [8]). Гладкая кубическая гиперповерхность $Q \subset \mathbb{P}^{4}$ нерациональна.

Доказательство этой теоремы использует сложную технику современной алгебраической геометрии; ниже мы скажем несколько слов о том направлении, которое выросло из замечательной работы Клеменса и Гриффитса.

Делая еще один шаг, а именно повышая размерность, мы получим уже открытую проблему: рациональна ли общая гладкая кубическая гиперповерхность $Q \subset \mathbb{P}^{n}$ при $n \geqslant 5$ ?

Предполагается, что ответ отрицательный при четном $n \in 2 \mathbb{Z}$, а при нечетном $n$ ответ отрицательный для гиперповерхности общего положения. Легко видеть, что при $n=2 k+1$ конструкция примера $1.1,(\mathrm{v})$ дает рациональность кубики в том случае, когда $Q$ содержит пару непересекающихся плоскостей $P_{ \pm}$ размерности $k$. Известно, однако, что при $n \geqslant 5$ на общей кубике $k$-плоскостей нет.

Проблема рациональности четномерной кубики интересна еще и потому, что связана со следующим вопросом: можно ли гладко продеформировать гладкое рациональное многообразие в нерациональное? Если общая четномерная кубика нерациональна, то в силу сказанного выше ответ на поставленный вопрос положительный. Проблеме рациональности кубической гиперповерхности размерности 4 и выше посвящено очень мало работ. Неясно даже, какой именно подход приведет к цели. Недавнее обсуждение этой проблемы в духе трансцендентного метода см. в [9].

Проблема рациональности, формулировка которой по своей простоте доступна продвинутому школьнику, важна по следующим причинам:

- рациональные многообразия в очевидном смысле являются "наиболее простыми" алгебраическими многообразиями, и их необходимо уметь отличать от остальных многообразий; 
- за проблемой рациональности стоит традиция, уходящая к истокам алгебраической геометрии;

- проблема рациональности обычно очень трудная - при условии, что она не допускает тривиального решения для данного класса многообразий: например, очевидной геометрической конструкции (типа стереографической проекции) или очевидного бирационального инварианта типа дифференциальных форм, о чем пойдет речь чуть ниже; альтернатива “либо очень просто, либо очень сложно" характерна для проблемы рациональности и примыкающих к ней вопросов;

- проблема рациональности важна с арифметической точки зрения, когда ищутся решения системы полиномиальных уравнений, определенные над полем рациональных чисел $\mathbb{Q}$ или его конечными расширениями: если многообразие параметризуемо, то параметрам можно придавать произвольные значения, принадлежащие заданному полю;

- проблема рациональности, важная сама по себе, порождает многие глубокие проблемы, имеющие более общий характер и независимый смысл; в частности проблема рациональности важна с точки зрения классификации алгебраических многообразий.

1.2. Бирациональная геометрия. Большинство отображений и соответствий, большинство естественных геометрических конструкций, возникающих в алгебраической геометрии, определены лишь в точках общего положения (например, стереографическая проекция не определена в точке, которая является центром проекции). Это приводит к концепции рационального отображения, т. е. отображения, определенного на открытом плотном подмножестве алгебраического многообразия. Произвольное отображение $\varphi: \mathbb{P}^{n} \rightarrow \mathbb{P}^{m}$ имеет вид

$$
\left(x_{0}: \ldots: x_{n}\right) \mapsto\left(f_{0}\left(x_{*}\right): \ldots: f_{m}\left(x_{*}\right)\right),
$$

где $f_{i}$ - однородные многочлены одной и той же степени $d \geqslant 1$, не имеющие общего нетривиального делителя. Очевидно, $\varphi$ не определено в точках замкнутого алгебраического множества $f_{0}\left(x_{*}\right)=\cdots=f_{m}\left(x_{*}\right)=0$, которое имеет коразмерность $\geqslant 2$ в силу сказанного о наборе многочленов $f_{*}$. Если это множество непусто, отображение $\varphi$ называется рациональным (в противном случае - регулярным). Прерывистая стрелка -- $\rightarrow$ означает, что отображение, вообще говоря, определено не всюду. Для регулярных отображений используется обычная стрелка $\rightarrow$. Важнейшая информация об отображении сосредоточена именно на том множестве, где оно не определено.

Центральным объектом изучения в алгебраической геометрии являются проективные многообразия, и концепция рационального отображения естественно обобщается до рационального отображения произвольных проективных многообразий $\varphi: X \rightarrow Y$. (В алгебраической терминологии рациональным доминантным отображениям $\varphi$ соответствуют вложения полей рациональных функций $\varphi^{*}: \mathbb{C}(Y) \hookrightarrow \mathbb{C}(X)$.) Обратимые рациональные отображения называются бирациональными отображениями. (На алгебраическом языке бирациональным отображениям $\varphi: X \rightarrow Y$ соответствуют изоморфизмы полей рациональных функций $\mathbb{C}(Y) \leftrightarrow \mathbb{C}(X)$.) Бирациональная классификация алгебраических 
многообразий (т. е. классификация с точностью до бирациональной эквивалентности) является наиболее разумной формой их классификации.

Основная трудность работы с (би)рациональными отображениями заключается в том, что каждое отображение имеет свою собственную область определения. Основной метод работы с (би)рациональными отображениями состоит в процедуре их регуляризации путем последовательности раздутий. Раздутия представляют собой простейшие бирациональные отображения, описанные в учебниках [4]-[7]. Раздутие начала координат $о \in \mathbb{C}^{n}$ определяется как отображение $\sigma: X \rightarrow \mathbb{C}^{n}$, где $X \subset \mathbb{C}^{n} \times \mathbb{P}^{n-1}$ есть замыкание множества $\left(\left(z_{1}, \ldots, z_{n}\right),\left(z_{1}: \ldots: z_{n}\right)\right)$ для $z \neq(0, \ldots, 0)$. Отображение $\sigma$ задает изоморфизм множеств $X \backslash \sigma^{-1}(o)$ и $\mathbb{C}^{n} \backslash\{o\}$, и $\sigma^{-1}(o)=\mathbb{P}^{n-1}$, т. е. вместо точки $о$ в $\mathbb{C}^{n}$ вклеивается гладкая гиперповерхность, изоморфная $\mathbb{P}^{n-1}$. Раздутие плоскости $\left\{z_{1}=\cdots=z_{n}=0\right\}=\{o\} \times \mathbb{C}^{m} \subset \mathbb{C}^{n+m}$ есть отображение $X \times \mathbb{C}^{m} \rightarrow \mathbb{C}^{n} \times \mathbb{C}^{m}=\mathbb{C}^{n+m} ;$ это дает идею определения раздутия произвольного гладкого подмногообразия $Y \subset V$.

Теперь, вместо того, чтобы интерпретировать рациональное отображение $\varphi: V \rightarrow W$ как регулярное отображение $\varphi: U \rightarrow W$ некоторого открытого плотного множества $U \subset V, \mathrm{k} V$ применяется последовательность раздутий

$$
V_{N} \stackrel{\sigma_{N}}{\longrightarrow} V_{N-1} \rightarrow \cdots \rightarrow V_{i+1} \stackrel{\sigma_{i+1}}{\longrightarrow} V_{i} \rightarrow \cdots \rightarrow V=V_{0}
$$

так, что сквозное отображение $\psi=\varphi \circ \sigma_{1} \circ \cdots \circ \sigma_{N}: V_{N} \rightarrow W$ определено всюду, т. е. является регулярным отображением (морфизмом) $\psi: V_{N} \rightarrow W$. При этом многообразия $V$ и $V_{N}$ бирационально эквивалентны. Описанная процедура называется разрешением особенностей (в классической терминологии, разрешением точек неопределенности) отображения $\varphi$. Теория разрешения особенностей является самостоятельной областью бирациональной геометрии. Разрешение особенностей в произвольной размерности впервые было доказано Х. Хиронакой [10], в последние пятнадцать лет появились более короткие и простые доказательства, проблема существования разрешения и его реализации в виде алгоритма рассматривалась во многих вариантах (см. [11] и библиографию в этой работе).

В общем контексте бирациональной классификации алгебраических многообразий проблема рациональности формулируется как вопрос о бирациональной эквивалентности заданного многообразия $V$ и проективного пространства $\mathbb{P}^{n}, n=\operatorname{dim} V$.

Проблема рациональности порождает многочисленные сопутствующие вопросы, примеры которых мы сейчас приведем.

- Для заданного многообразия $V$ : существует ли рациональная функция $f: V \rightarrow \mathbb{C}$, общий слой которой $f^{-1}(a), a \in \mathbb{C}$, есть рациональное многообразие? И если такая функция существует, влечет ли это рациональность самого многообразия $V$ ?

- В обобщение предыдущего вопроса, существует ли для заданного многообразия $V$ рациональное доминантное (т. е. покрывающее открытое плотное множество на образе) отображение $\varphi: V \rightarrow S$, слой общего положения которого $\varphi^{-1}(s), s \in S$, рационален? И снова, если ответ 
положителен и отображение $\varphi$ с такими свойствами существует, а многообразие $S$ рационально, влечет ли это рациональность $V$ ?

- С проблемой рациональности тесно связана задача описания группы бирациональных автоморфизмов $\operatorname{Bir} V$ многообразия $V$.

- Проблема рациональности обобщается до проблемы унирациональности: для заданного многообразия $V$ существует ли рациональное доминантное отображение $\mathbb{P}^{n} \rightarrow V$ (если такое отображение существует, то многообразие $V$ называется унирациональным)? Иными словами, речь идет о многозначной параметризации многообразия $V$. (На алгебраическом языке эта проблема формулируется следующим образом: верно ли, что любое подполе $F \subset \mathbb{C}\left(t_{1}, \ldots, t_{n}\right)$ поля рациональных функций, $\operatorname{tr} . \operatorname{deg} F / \mathbb{C}=n$, само есть поле рациональных функций от $n$ независимых переменных, $F \cong \mathbb{C}\left(t_{1}, \ldots, t_{n}\right)$ ?) Вопрос о том, влечет ли унирациональность рациональность, называется проблемой Люрота; более подробно речь о ней пойдет ниже.

Эти и многие другие проблемы для “большинства" алгебраических многообразий тривиальны, как и сама проблема рациональности. Для гладкого проективного многообразия $V$ рассмотрим его кокасательное расслоение $T^{*} V$. Пусть $E$ - некоторое векторное расслоение над $V$, полученное из $T^{*} V$ применением следующих операций: взятия симметрической или кососимметрической степени, тензорного произведения и тензорной степени (например, $\left(T^{*} V\right)^{\otimes n} \otimes$ $\left.\left(\Lambda^{k} T^{*} V\right)^{l}\right)$. По сути, исключается только операция дуализации: $E$ должно быть строго ковариантным тензорным объектом. Пусть $s \in H^{0}(V, E)$ - ненулевое глобальное сечение расслоения $E$. Тогда для любого рационального доминантного отображения $\beta: W \rightarrow V$ подъем $\beta^{*} s$ сечения $s$ продолжается до ненулевого глобального сечения расслоения $F$, полученного из кокасательного расслоения $T^{*} W$ применением идентичного набора тензорных операций. Например, подъем $\beta^{*} \omega$ регулярной дифференциальной формы степени $i, \omega \in H^{0}\left(V, \Omega_{V}^{i}\right)$ (где $\Omega_{V}^{i}$ есть пучок сечений векторного расслоения $\Lambda^{i} T^{*} V$ ), есть регулярная форма степени $i$ на $W$. Это связано с упомянутым выше фактом: рациональное отображение $\beta$ регулярно на открытом множестве $W \backslash Z$, где $Z \subset W$ имеет коразмерность $\geqslant 2$, так что регулярная форма $\beta^{*} \omega$ продолжается с $W \backslash Z$ на все $W$.

Следовательно, если на $V$ имеется хотя бы один регулярный ковариантный тензор, то многообразие $V$ нерационально, поскольку, как нетрудно проверить, на проективном пространстве таких тензоров нет (например, все дифференциальные формы на $\mathbb{P}^{n}$ имеют гиперповерхности полюсов).

Пример 1.2. Хорошо известно, что гладкая кубическая кривая $C \subset \mathbb{P}^{2}$ изоморфна комплексному тору $\mathbb{C} / \Lambda$, где $\Lambda \cong \mathbb{Z} \oplus \mathbb{Z}-$ некоторая решетка. Если $z-$ комплексная координата на $\mathbb{C}$, то пространство $H^{0}\left(C, \Omega_{C}\right)$ глобальных регулярных (т. е. голоморфных) форм на $C$ одномерно и имеет вид $\{\lambda d z \mid \lambda \in \mathbb{C}\}$. Следовательно, кривая $C$ нерациональна.

ПримеР 1.3. Согласно классической формуле присоединения, пространство регулярных дифференциальных форм максимальной степени на гладкой гиперповерхности $V=V_{d} \subset \mathbb{P}^{N}$ при $d \geqslant N+1$ изоморфно пространству многочле- 
нов степени $(d-N-1)$ от однородных координат $x_{0}, \ldots, x_{N}$, откуда немедленно следует, что $V$ нерациональна. На самом деле, можно сказать гораздо больше: при $d \geqslant N+2$ регулярных форм максимальной степени на $V$ "очень много", пучок сечений расслоения $\Lambda^{N-1} T^{*} V$ очень обилен (нули сечений разделяют точки и касательные направления), так что бирациональная геометрия этих многообразий полностью сводится к их проективной геометрии. Например, две гладкие гиперповерхности $V_{1}$ и $V_{2}$ степеней $d_{1}, d_{2} \geqslant N+2$ бирационально эквивалентны тогда и только тогда, когда $d_{1}=d_{2}$ и $V_{1}$ проективно эквивалентна $V_{2}$, т. е. переводится в $V_{2}$ проективным автоморфизмом, индуцированным некоторым линейным автоморфизмом пространства $\mathbb{C}^{N+1}$.

Поэтому наибольшие трудности в изучении бирациональной геометрии алгебраических многообразий возникают именно там, где нет ненулевых глобальных дифференциальных форм (и, более общо, глобальных ковариантных тензоров) или, по крайней мере, там, где их мало. Именно для таких многообразий проблема рациональности является нетривиальной (и, как правило, очень трудной). Например, таковы гиперповерхности малой степени $V_{d} \subset \mathbb{P}^{N}$, $d \leqslant N$, и, более общо, полные пересечения $V_{d_{1} \cdots d_{k}}=F_{d_{1}} \cap \cdots \cap F_{d_{k}} \subset \mathbb{P}^{N}$ гиперповерхностей $F_{d_{1}}, \ldots, F_{d_{k}}$ степеней $d_{1}, \ldots, d_{k}$, удовлетворяющих неравенству $d_{1}+\cdots+d_{k} \leqslant N$. Крайний случай - само проективное пространство $\mathbb{P}^{N}$; для него проблема рациональности не стоит, однако оно не выпадает из общего ряда: его бирациональная геометрия является наиболее богатой и сложной.

1.3. Две классические теоремы. В размерностях 1 и 2 дифференциально-геометрические инварианты полностью решают проблему рациональности. Это было осознано уже классическими геометрами. Кривая $C$ рациональна тогда, когда ее род $g_{C}=\operatorname{dim} H^{0}\left(C, \Omega_{C}^{1}\right)$ равен нулю. В частности, понятия унирациональности и рациональности для кривых совпадают. Для поверхностей ситуация аналогична (однако в размерности 2 уже существенно, что поле констант алгебраически замкнуто: для незамкнутых полей геометрия существенно сложнее, см. [12], [13]). А именно, справедлива следующая теорема.

ТеоремА 1.2 (критерий рациональности Кастельнуово). Пусть $S$ - гладкая проективная поверхность, удовлетворяющая условиям

$$
H^{0}\left(S, \Omega_{S}^{1}\right)=H^{0}\left(S, \omega_{S}^{\otimes 4}\right)=H^{0}\left(S, \omega_{S}^{\otimes 6}\right)=\{0\},
$$

где $\omega_{S}=\Lambda^{2} \Omega_{S}-$ пучок форм максимальной степени. Тогда $S$ рачиональна.

Поскольку все дифференциально-геометрические инварианты унирационального многообразия тривиальны, критерий рациональности Кастельнуово положительно решает проблему Люрота в размерности два: унирациональные поверхности рациональны. Таким образом, нетривиальная бирациональная геометрия поверхностей сосредоточена (главным образом) на единственном объекте: проективной плоскости $\mathbb{P}^{2}$. Рациональные поверхности имеют очень богатую геометрию. Мы коснемся здесь лишь нескольких вопросов, важных для дальнейшего.

Одним из наиболее ярких результатов двумерной бирациональной геометрии является следующая теорема М. Нётера [14]. 
Теорема 1.3. Группа бирачиональных автоморфизмов проективной плоскости $\operatorname{Bir} \mathbb{P}^{2}=\left\{\chi: \mathbb{P}^{2} \rightarrow \mathbb{P}^{2}\right\}$ порождена подгруппой проективных автоморфизмов Aut $\mathbb{P}^{2}=\mathrm{PGL}(2)$ и единственным квадратичным преобразованием

$$
\tau:\left(x_{0}: x_{1}: x_{2}\right) \mapsto\left(x_{1} x_{2}: x_{0} x_{2}: x_{0} x_{1}\right) .
$$

Бирациональные автоморфизмы проективного пространства часто называют кремоновыми преобразованиями, а группу Bir $\mathbb{P}^{n}-n$-мерной группой Кремоны, так что сформулированный выше факт более всего известен как "теорема Нётера о группе Кремоны".

Опишем общую идею доказательства теоремы 1.3 .

Пусть $\chi: \mathbb{P}^{2} \rightarrow \mathbb{P}^{2}-$ произвольный бирациональный автоморфизм. Будем модифицировать его, рассматривая композицию $\chi \circ \beta_{1} \circ \beta_{2} \circ \cdots \circ \beta_{k}=\chi_{k}$, где $\beta_{i}$ - либо линейный автоморфизм, т. е. элемент группы $\operatorname{Aut} \mathbb{P}^{2}=\mathrm{GL}(3, \mathbb{C}) / \mathbb{C}^{*}=$ $\operatorname{PGL}(2, \mathbb{C})$, либо инволюция $\tau$. Необходимо так построить процедуру модификации, чтобы на каком-то шаге получить $\chi_{N} \in$ Aut $\mathbb{P}^{2}$.

Предположим, что $\chi \notin A u t \mathbb{P}^{2}$ (в противном случае модифицировать нечего). По определению, существует конечное множество точек $Y \subset \mathbb{P}^{2}$ такое, что $\left.\chi\right|_{\mathbb{P}^{2} \backslash Y}$ есть регулярное отображение в $\mathbb{P}^{2}$. Определим собственный прообраз $\left(\chi^{-1}\right)_{*} C$ кривой $C \subset \mathbb{P}^{2}$ относительно $\chi$ как замыкание в $\mathbb{P}^{2}$ множества

$$
\left(\left.\chi\right|_{\mathbb{P}^{2} \backslash Y}\right)^{-1}(C) \subset \mathbb{P}^{2} \backslash Y \text {. }
$$

Процедура замыкания сводится к добавлению точек из конечного множества $Y$. Рассмотрим теперь линейную систему прямых в $\mathbb{P}^{2}$, т. е. множество всех прямых $a_{0} x_{0}+a_{1} x_{1}+a_{2} x_{2}=0$ с $\left(a_{0}, a_{1}, a_{2}\right) \neq(0,0,0)$, и построим ее собственный прообраз относительно $\chi$. Получим линейную систему кривых $|\chi|$ некоторой степени $n=n(\chi) \geqslant 1$, т. е. множество кривых, заданных уравнением

$$
a_{0} f_{0}\left(x_{*}\right)+a_{1} f_{1}\left(x_{*}\right)+a_{2} f_{2}\left(x_{*}\right)=0,
$$

где $f_{i}$ - многочлен степени $n$, причем для общего набора коэффициентов $a_{*}$ многочлен $\sum a_{i} f_{i}$ неприводим. Собственно, отображение $\chi$ задается в системе однородных координат $x_{*}$ именно этими многочленами:

$$
\chi:\left(x_{0}: x_{1}: x_{2}\right) \mapsto\left(f_{0}\left(x_{*}\right): f_{1}\left(x_{*}\right): f_{2}\left(x_{*}\right)\right) .
$$

Легко понять, что многочлены $f_{i}$ не могут быть произвольными: по теореме Безу две кривые $C_{1}, C_{2} \in|\chi|$ пересекаются в $n^{2}$ точках, в то время как соответствующие им прямые $L_{1}, L_{2}$ трансверсально пересекаются в одной точке. Можно утверждать, что кривые $C_{1}, C_{2}$ трансверсально пересекаются в одной точке вне исключительного множества $Y$. Поэтому при $n \geqslant 2$ (а если $n=1$, то $\chi \in$ Aut $\mathbb{P}^{2}$ - проективный автоморфизм) множество $Y$ непусто и в каждой точке $y \in Y$ общий многочлен $\sum a_{i} f_{i}$ имеет некоторую положительную кратность $m(y) \geqslant 1$. Раздувая конечное множество $Y$, получаем некоторую рациональную поверхность $S$, снабженную регулярным бирациональным отображением $\sigma: S \rightarrow \mathbb{P}^{2}$, где $\sigma: S \backslash \sigma^{-1}(Y) \rightarrow \mathbb{P}^{2} \backslash Y$ взаимно однозначно, а в каждую точку $y \in Y$ стягивается гладкая рациональная кривая. 
Рассмотрим собственный прообраз $\left(\sigma^{-1}\right)_{*}|\chi|$ линейной системы $|\chi|$ на $S$ : это линейная система кривых на $S$, общая кривая которой получается из общей кривой $C \in|\chi|$ в два шага: сначала берется прообраз $\sigma^{-1}(C \backslash Y)$, затем он замыкается в $S$. Может случиться, что сквозное отображение $\chi \circ \sigma: S \rightarrow \mathbb{P}^{2}$ имеет точки неопределенности, т. е. в точках конечного множества $Y_{1} \subset S_{1}=S$ отображение $\chi \circ \sigma$ нерегулярно. В этом случае все кривые системы $\left(\sigma^{-1}\right)_{*}|\chi|$ проходят через каждую точку множества $Y_{1}$, которое называется базисным множеством данной линейной системы (обозначение: $\operatorname{Bs}\left(\sigma^{-1}\right)_{*}|\chi| ;$ например, $Y=\operatorname{Bs}|\chi|)$. Если $Y_{1} \neq \varnothing$, то описанную процедуру необходимо повторить. В результате получаем последовательность раздутий

$$
S_{N} \stackrel{\sigma_{N}}{\longrightarrow} S_{N-1} \rightarrow \cdots \rightarrow S_{i} \stackrel{\sigma_{i}}{\longrightarrow} S_{i-1} \rightarrow \cdots \stackrel{\sigma_{1}=\sigma}{\longrightarrow} S_{0}=\mathbb{P}^{2},
$$

разрешающих особенности линейной системы $|\chi|$, т. е. ее собственный прообраз не имеет базисных точек, а сквозное отображение $\chi \circ \sigma_{1} \circ \cdots \circ \sigma_{N}: S_{N} \rightarrow \mathbb{P}^{2}$ регулярно. Теперь две кривые $C_{1}, C_{2}$ из линейной системы $\left(\sigma_{1} \circ \cdots \circ \sigma_{N}\right)_{*}^{-1}|\chi|$ трансверсально пересекаются в одной точке уже без всяких оговорок.

Отображение $\sigma_{i}: S_{i} \rightarrow S_{i-1}$ раздувает конечное множество точек $Y_{i-1} \subset$ $S_{i-1}$, элементы которого при $i \geqslant 2$ называются бесконечно близкими точками $(i-1)$-го порядка. Каждой точке $y \in Y_{i} \subset S_{i}$ соответствует целое положительное число $m(y) \geqslant 1$ - ее кратность относительно общей кривой системы $\left(\sigma_{1} \circ \cdots \circ \sigma_{i}\right)_{*}^{-1}|\chi|$. Теперь условие, что две различные прямые пересекаются трансверсально в одной точке, дает равенство

$$
n^{2}=1+\sum_{j=1}^{K} m_{j}^{2}
$$

где $m_{j}=m\left(y_{j}\right)$, а $\left\{y_{j} \mid j=1, \ldots K\right\} \subset \bigvee_{i=0}^{N-1} Y_{i}-$ множество всех базисных точек, включая бесконечно близкие.

Равенство (2) есть первое ограничение на линейную систему $|\chi|$, т. е. на многочлены $f_{i}$. Второе ограничение мы получим, вспоминая, что кривые $C \in|\chi|$ все рациональны: $\chi: C \rightarrow \mathbb{P}^{1}$ есть бирациональное отображение. Собственный прообраз $\widetilde{C}$ кривой $C$ на $S_{N}$ есть гладкая рациональная кривая. Классическая формула для рода кривой дает равенство

$$
\frac{(n-1)(n-2)}{2}=\sum_{j=1}^{K} \frac{m_{j}\left(m_{j}-1\right)}{2}
$$

Оставляем читателю в качестве элементарного упражнения доказательство следующего факта.

Предложение 1.1 (неравенство Клебша-Нётера). Если $n \geqslant 2, m o K \geqslant 3$ и имеет место неравенство

$$
m_{1}+m_{2}+m_{3}>n
$$

(где предполагается, что кратности $m_{j}$ упорядочень: $m_{1} \geqslant m_{2} \geqslant \cdots \geqslant m_{j} \geqslant$ $\left.m_{j+1} \geqslant \cdots\right)$. 
Предположим, что имеет место случай общего положения: три наибольшие кратности $m_{1}, m_{2}, m_{3}$ соответствуют трем различным точкам множества $Y$ (к сожалению, это далеко не всегда так и может случиться, что уже $y_{2}-$ бесконечно близкая точка над $\left.y_{1}\right)$. Легко понять, что $y_{1}, y_{2}, y_{3}$ не коллинеарны: в противном случае в силу неравенства Клебша-Нётера для общей кривой $C \in|\chi|$ и прямой $L \ni y_{i}$ мы имели бы

$$
n=(C \cdot L) \geqslant \sum_{j=1}^{3}(C \cdot L)_{y_{j}} \geqslant m_{1}+m_{2}+m_{3}>n,
$$

что невозможно (учтем, что общая кривая $C$ неприводима и не может содержать прямую $L$ компонентой). Пусть $\alpha \in$ Aut $\mathbb{P}^{2}$ - проективное преобразование, переводящее точки $y_{1}, y_{2}, y_{3}$ в точки $(1: 0: 0),(0: 1: 0)$ и $(0: 0: 1)$ соответственно. Рассмотрим композицию

$$
\chi_{1}=\chi \circ\left(\alpha^{-1} \circ \tau \circ \alpha\right): \mathbb{P}^{2} \rightarrow \mathbb{P}^{2} .
$$

ПреДЛОЖенИЕ 1.2. Линейная система $|\chi|$ состоит из кривых степени $n\left(\chi_{1}\right)=2 n(\chi)-m_{1}-m_{2}-m_{3}<n(\chi)$.

ДокАЗАтельСтво. По построению отображения $\chi_{1}$, общая кривая $C^{+} \in\left|\chi_{1}\right|$ есть собственный прообраз общей кривой $C \in|\chi|$ относительно квадратичного отображения $\alpha^{-1} \circ \tau \circ \alpha$ :

$$
\begin{gathered}
\mathbb{P}^{2} \stackrel{\alpha^{-1} \tau \alpha}{\rightarrow} \mathbb{P}^{2}-{ }^{\chi}->\mathbb{P}^{2} \\
\cup \\
\cup \\
C^{+}<-
\end{gathered}
$$

Степень кривой $C^{+}$равна индексу пересечения $\left(C^{+} \cdot L\right)$, где $L \subset \mathbb{P}^{2}$ - общая прямая. Очевидно, индекс пересечения $\left(C^{+} \cdot L\right)$ равен индексу свободного пересечения кривой $\alpha^{-1} \tau \alpha\left(C^{+}\right)=C$ и коники $Q=\alpha^{-1} \tau \alpha(L)$, т. е. индексу пересечения вне точек неопределенного отображения $\alpha^{-1} \tau \alpha$ (отметим, что последнее инволютивно). Итак,

$$
\left(C^{+} \cdot L\right)+\sum_{j=1}^{3}(C \cdot Q)_{y_{j}}=(C \cdot Q)=2 n,
$$

откуда и следует наше утверждение.

Описанная процедура перехода от $\chi$ к $\chi_{1}$ называется откручиванием тройки точек $\left(y_{1}, y_{2}, y_{3}\right)$. Как отмечалось выше, откручивание корректно определено, если среди точек $y_{*}$ нет бесконечно близких. В первоначальном рассуждении Нётера [14] возможность существования бесконечно близких точек не учитывалась, и потребовалось несколько десятилетий, чтобы научиться откручивать наборы точек, включающие бесконечно близкие точки. Современные доказательства см. в [15]-[17]. Однако все доказательства опираются на описанную идею Нётера: беря композицию с подходящим "элементарным" бирациональным отображением, упрощать заданное отображение $\chi$. Процесс упрощения 
контролируется целочисленными инвариантами (в нашем случае - степенью $n(\chi) \geqslant 1)$ и потому не может быть бесконечным. На каком-то шаге получим

$$
\chi \circ \alpha_{1} \circ \tau \circ \alpha_{2} \circ \tau \circ \cdots \circ \alpha_{N-1} \circ \tau \circ \alpha_{N} \in \operatorname{Aut} \mathbb{P}^{2},
$$

откуда и следует теорема Нётера.

Существует огромная литература по теореме Нётера и преобразованиям Кремоны. Бирациональные преобразования плоскости (преобразования Кремоны) являются одновременно очень естественным и очень нетривиальным объектом, порождающим множество задач (описать с точностью до сопряжения инволюции в группе Кремоны, описать конечные подгруппы [18], описать подгруппу, оставляющую инвариантной некоторый геометрический объект например кривую, и т.д.). Бирациональные преобразования в размерности три намного сложнее и разнообразнее. Не удивительно, что на рубеже XIX и XX столетий им были посвящены сотни статей - см. библиографию в [19]. И сейчас изучение различных конкретных семейств бирациональных преобразований проективного пространства является весьма популярной темой. Однако предметом настоящего обзора являются как раз нерациональные многообразия. Возвращаясь к теореме Нётера, перечислим основные ингредиенты его рассуждения:

- инвариант $n=n(\chi) \geqslant 1$, измеряющий сложность бирационального отображения (степень кривых в линейной системе $|\chi|)$,

- "максимальные" тройки базисных точек линейной системы $|\chi|$ (удовлетворяющие неравенству Нётера),

- процедура откручивания, уменьшающая инвариант $n$ и упрощающая отображение $\chi$.

Отметим следующее важное обстоятельство: рассуждение Нётера остается в силе, если в качестве линейной системы $|\chi|$ рассматривать произвольный пучок рациональных кривых, т. е. линейную систему вида $a_{0} f_{0}\left(x_{*}\right)+a_{1} f_{1}\left(x_{*}\right)=0$ (с условием, что общая кривая рациональна). Дословное повторение доказательства теоремы Нётера дает следующий важный факт.

Теорема 1.4. Любой пучок рациональных кривых на $\mathbb{P}^{2}$ можно перевести в пучок прямых $a_{0} x_{0}+a_{1} x_{1}=0$ некоторым преобразованием Кремоны.

Далее, из приведенных выше рассуждений ясно, что соотношения между образующими группы Кремоны появляются тогда и только тогда, когда процедура откручивания определена не однозначно. Такое действительно возможно. На этом пути соотношения в двумерной группе Кремоны были описаны М. Х. Гизатуллиным [20]. Позже его доказательство было радикально упрощено В. А. Исковских [21].

Упомянем, наконец, еще один классический факт теории алгебраических поверхностей.

ТЕОРЕма 1.5. Если на гладкой проективной поверхности $S$ имеется пучок рациональных кривых, то $S$ рачиональна.

Доказательство можно найти в стандартных учебниках, см., например, [22].

Подытожим картину двумерной бирациональной геометрии (созданной, главным образом, М. Нётером и итальянскими геометрами): 
- унирациональность влечет рациональность;

- на поверхности $S$ тогда и только тогда существует рациональная функция $f: S \rightarrow \mathbb{C}$, общий слой $f^{-1}(a), a \in \mathbb{C}$, которой есть рациональная кривая, когда ее можно дополнить такой рациональной функцией $g: S \rightarrow \mathbb{C}$, что пара $(f, g): S \rightarrow \mathbb{C}^{2}$ задает бирациональное отображение;

- вопрос о рациональности поверхности $S$ полностью решается в терминах ее дифференциально-геометрических инвариантов;

- группа Кремоны Bir $\mathbb{P}^{2}$ порождается автоморфизмами Aut $\mathbb{P}^{2}$ и одним квадратичным преобразованием.

Многомерная бирациональная геометрия началась с попыток перенести эти результаты в размерность три.

\section{§ 2. Первые шаги современной бирациональной геометрии}

2.1. Работы Фано. Джино Фано занимает промежуточное положение в истории алгебраической геометрии. Он был последним выдающимся представителем старой итальянской школы, главным достижением которой считается теория алгебраических поверхностей, однако предметом его исследований были трехмерные алгебраические многообразия. Если с поверхностями еще можно работать на достаточно элементарном уровне, апеллируя при необходимости к геометрической интуиции, то в более высоких размерностях требуется систематическая разработка оснований, интуитивно правдоподобные утверждения могут оказаться ошибочными. Пучки и когомологии, теория пересечений, общая теорема Римана-Роха-Хирцебруха-Гротендика, разрешение особенностей - все это появилось в алгебраической геометрии уже после Фано. Однако, несмотря на то, что в его работах немало ошибочных рассуждений и, по существу, ни одна из его больших теорем не была им доказана, Фано выдвинул ряд глубоких и плодотворных идей и угадал ряд фундаментальных фактов трехмерной алгебраической геометрии [23]-[25].

Фано начал с попытки воспроизвести рассуждения Нётера в размерности три. Первым объектом его исследований была трехмерная квартика $V=$ $V_{4} \subset \mathbb{P}^{4}$. На гладкой трехмерной квартике нет ненулевых глобальных регулярных дифференциальных форм и, более общо, ковариантных тензоров, так что в терминах дифференциальной геометрии решить проблему рациональности квартики не удается. Фано стремился описать группу бирациональных автоморфизмов квартики $\operatorname{Bir} V$. Рассуждая по схеме Нётера, для бирационального автоморфизма $\chi: V \rightarrow V$ он рассмотрел собственный прообраз линейной системы гиперплоских сечений $V$ относительно $\chi$ :

$$
\begin{gathered}
V------^{\chi}---->V \\
\left\{\begin{array}{c}
\text { линейная } \\
\text { система } \\
\text { дивизоров }|\chi| \text { на } V
\end{array}\right\} \stackrel{\left(\chi^{-1}\right)_{*}}{<}\left\{\begin{array}{c}
\text { линейная система } \\
\text { гиперплоских } \\
\text { сечений }
\end{array}\right\}
\end{gathered}
$$


Полученная линейная система дивизоров высекается на $V$ гиперповерхностями степени $n=n(\chi) \geqslant 1$ в $\mathbb{P}^{4}$. По аналогии с двумерным случаем, мы получаем следующую альтернативу: либо $n=1$ и в этом случае $\chi \in$ Aut $V$ есть регулярный автоморфизм (гиперплоские сечения переводятся в гиперплоские сечения, так что $\chi$ есть ограничение $\chi=\left.\widehat{\chi}\right|_{V}$ некоторого проективного автоморфизма $\hat{\chi} \in \operatorname{Aut} \mathbb{P}^{4}$, сохраняющего $V$ ), либо $n \geqslant 2$ и в этом случае отображение $\chi$ нерегулярно, в частности, базисное множество Bs $|\chi|$ непусто.

Фано утверждал, что случай $n \geqslant 2$ невозможен. По аналогии с двумерным случаем, при $n \geqslant 2$ в базисном множестве Bs $|\chi|$ необходимо найти подмножество высокой кратности (аналог тройки точек $\left.y_{1}, y_{2}, y_{3}\right)$. Рассуждая не вполне формально, Фано пришел к выводу, что при $n \geqslant 2$ имеет место одна из следующих возможностей:

- существует точка неопределенности $x \in \mathrm{Bs}|\chi|$ такая, что mult $x|\chi|>2 n$;

- существует целая кривая $B \subset \mathrm{Bs}|\chi|$ точек неопределенности такая, что $\operatorname{mult}_{B}|\chi|>n$;

- существует подмножество высокой кратности, состоящее из бесконечно близких точек.

Объясним, что здесь имеется в виду: сам Фано не дал (в силу неразработанности оснований, вероятно, и не мог дать) точного описания, ограничившись примером, который мы и воспроизведем. Этот пример аналогичен ситуации, когда в доказательстве теоремы Нётера среди точек $y_{1}, y_{2}, y_{3}$ оказывались бесконечно близкие. Итак, в примере Фано существуют точка $x \in \operatorname{Bs}|\chi|$ и целая прямая $B$ бесконечно близких точек над $x$, т. е. прямая $B \subset E \cong \mathbb{P}^{2}$ в исключительном дивизоре $E \subset \widetilde{V}$ раздутия $\varphi: \widetilde{V} \rightarrow V$ точки $x$, такие, что выполнено неравенство

$$
\operatorname{mult}_{x}|\chi|+\operatorname{mult}_{B}|\widetilde{\chi}|>3 n,
$$

где $|\tilde{\chi}|$ - собственный прообраз линейной системы $|\chi|$ на $\widetilde{V}$.

Подчеркнем, что последний пример лишь дает некоторое представление о бесконечно близком случае, не более того. Чтобы охватить все возможности, нужен специальный формализм, которого у Фано не было и который был построен в пионерской работе В.А. Исковских и Ю. И. Манина [3], обсуждаемой ниже.

Вернемся к рассуждениям Фано. Рассматривая линейные системы на квартике, он пришел к выводу, что ни одна из перечисленных трех возможностей не реализуется. (Отметим, что часть его аргументов была ошибочной, однако итоговый вывод правильный.) Отсюда следует, что случай $n \geqslant 2$ невозможен, так что группы бирациональных и бирегулярных автоморфизмов квартики совпадают: $\operatorname{Bir} V=\operatorname{Aut} V$. Последняя группа конечна, в общем случае тривиальна, откуда немедленно следует нерациональность квартики $V_{4} \subset \mathbb{P}^{4}$.

Вывод Фано показывал, что трехмерная бирациональная геометрия гораздо сложнее, чем геометрия поверхностей. Объектом изучения для него были трехмерные многообразия, аналогичные по своим свойствам квартике. Изучая гладкое полное пересечение квадрики и кубики $V=V_{2 \cdot 3} \subset \mathbb{P}^{5}$, Фано обнаружил, что, в отличие от квартики, некоторые линейные системы $|\chi|$ на $V$, высекаемые гиперповерхностями степени $n \geqslant 2$ в $\mathbb{P}^{5}$, могут иметь базисную кривую $B$ кратности mult $_{B}|\chi|>n$. Это может случиться, если $B=L-$ прямая 
в $\mathbb{P}^{5}$ (Фано пропустил другую возможность, когда $B$ есть коника специального типа, см. ниже $\S 3$ главы 2). Если этот случай реализуется, то, по аналогии с доказательством теоремы Нётера, существует процедура откручивания прямой $L$. Опишем ее кратко для полноты картины.

Легко видеть, что проекция из прямой $L$ задает рациональное доминантное отображение $\pi: V \rightarrow \mathbb{P}^{3}$. Его степень в общей точке равна 2: слой $\pi^{-1}(x)$, $x \in \mathbb{P}^{3}$, есть пересечение $V \cap P$ с соответствующей точке $x$ плоскостью $P \supset L$, $P \cong \mathbb{P}^{2}$, за вычетом самой прямой $L$. Но $V=F_{2} \cap F_{3}$ есть полное пересечение квадрики и кубики, так что $F_{2} \cap P=L \cup L_{P}$ и $F_{3} \cap P=L \cup Q_{P}$, где $L_{P}$ и $Q_{P}-$ прямая и коника соответственно. Таким образом, $\pi^{-1}(x)=L_{P} \cap Q_{P}$, как и утверждалось. Пусть $\tau_{L} \in \operatorname{Bir} V$ - инволюция Галуа, переставляющая точки в слоях проекции $\pi$. Легко подсчитать, что линейная система $\left|\tau_{L}\right|$ высекается на $V$ квартиками и mult $_{L}\left|\tau_{L}\right|=5$. Беря композицию $\chi \circ \tau_{L}: V \rightarrow V$, получаем линейную систему $\left|\chi \circ \tau_{L}\right|$, высекаемую на $V$ гиперповерхностями степени $4 n-3$ mult $_{L}|\chi|<n$, что определяет индуктивную процедуру откручивания и в конце концов позволяет описать группу бирациональных автоморфизмов $\operatorname{Bir} V_{2 \cdot 3}$. Откручивание для полного пересечения $V_{2 \cdot 3}$ детально рассмотрено ниже, в $§ 3$ главы 2 .

Таким образом, Фано нашел аналоги для всех основных ингредиентов метода Нётера:

- инвариант $n \geqslant 1$, измеряющий сложность бирационального отображения,

- подмножества высокой кратности в базисном множестве Bs $|\chi|$, включая бесконечно близкие точки на уровне описательных примеров,

- процедура откручивания или исключения подмножеств высокой кратности, упрощающая отображение $\chi$ через понижение инварианта $n$.

По-видимому, основные усилия Фано в области бирациональной геометрии были направлены на изучение трехмерной кубики $V=V_{3} \subset \mathbb{P}^{4}$. Описание группы бирациональных автоморфизмов $\operatorname{Bir} V$ пока недоступно даже для современных "высокотехнологичных" методов, однако Фано пытался его получить на уровне своих явных геометрических конструкций и интуитивно правдоподобных рассуждений, что, конечно, было нереально. Особый интерес к кубике был связан с тем, что кубика очевидным образом унирациональна (см. ниже п. 2.2), так что доказательство ее нерациональности сразу дает контрпример к проблеме Люрота, которая оставалась одной из главных проблем алгебраической геометрии вплоть до 1970 г., когда она была (отрицательно) решена.

Отметим, что геометрическая интуиция всегда вела Фано в правильном направлении, независимо от того, насколько корректными были его доказательства.

Изучая расслоения на коники (см. ниже п. 2.3 о теореме Саркисова), Фано пришел к выводу, что общее многообразие этого типа неунирационалъно. Вопрос об унирациональности рационального связного многообразия до сих пор за пределами возможностей: кроме тех случаев, когда унирациональность можно доказать явно прямой конструкцией, проблема унирациональности остается открытой. Однако общее мнение поддерживает предположение Фано. 
Недавно появились новые свидетельства в пользу неунирациональности некоторых многомерных многообразий [26]. Этот пример вместе с описанными конструкциями опережали свое время: многие прогнозы Фано остаются не доказанными и не опровергнутыми по сей день.

За рамками настоящего обзора остается "бирегулярная" компонента исследований Фано, а именно классификация трехмерных алгебраических многообразий с отрицательным (т. е. антиобильным) каноническим классом, которые называются теперь многообразиями Фано (квартика, кубика, полное пересечение $V_{2 \cdot 3} \subset \mathbb{P}^{5}$ - все принадлежат к этому типу многообразий). Как и в бирациональном направлении, результаты Фано не были полными, а их доказательства законченными, однако к сегодняшнему дню программа Фано полностью реализована [27] в работах Исковских, Шокурова, Мори, Мукая, Каваматы, Мияоки. Эта программа положила начало целой области современной алгебраической геометрии - программе минимальных моделей (в размерности три это теория Мори), см. [28]-[31] и библиографии в этих работах.

2.2. Решение проблемы Люрота. Современный этап бирациональной геометрии начался с работ Клеменса-Гриффитса [8] (о нерациональности гладкой трехмерной кубики $V_{3} \subset \mathbb{P}^{4}$ ) и Исковских-Манина [3] (о нерациональности гладкой трехмерной квартики $\left.V_{4} \subset \mathbb{P}^{4}\right)$. Поскольку кубика очевидным образом унирациональна, а также известны примеры унирациональных гладких квартик (принадлежащие Сегре), в работах [3], [8] была отрицательно решена проблема Люрота в размерности три. Вскоре последовала работа Артина-Мамфорда [32] с примерами нерациональных унирациональных многообразий произвольной размерности.

Напомним конструкцию унирациональности гладкой трехмерной кубики $V=V_{3} \subset \mathbb{P}^{4}$. Пусть $x \in V$ - точка общего положения, $T(x) \subset \mathbb{P}^{4}-$ гиперплоскость, касательная к $V$ в точке $x$. Общая прямая $L \subset T(x), L \ni x$, имеет в точности двукратное пересечение с $V$ в точке $x,(L \cdot V)_{x}=2$, так что $(V \circ L)=2 x+p(L)$, где $p(L) \in L$ - третья точка пересечения $V$ с $L$. Здесь символ о обозначает теоретико-схемное пересечение прямой $L$ и гиперповерхности $V$ в $\mathbb{P}^{4}$, т. е. пересечение с учетом кратности каждой неприводимой компоненты. Таким образом, получаем рациональное отображение

$$
p_{x}: \mathbb{T}(x) \leftrightarrow--\rightarrow V \cap T(x),
$$

где $\mathbb{T}(x)=\mathbb{P}\left(T_{x} V\right) \cong \mathbb{P}^{2}$ - множество прямых $L \subset T(x)$, содержащих точку $x$. На самом деле $p$ бирационально и устанавливает рациональность кубической поверхности $V \cap T(x)$ с двойной точкой $x$. Рассмотрим теперь произвольную рациональную кривую $C \subset V$ (например, прямую). Поскольку касательное расслоение локально тривиально, имеем бирациональную эквивалентность

$$
\bigcup_{x \in C} \mathbb{T}(x) \rightarrow C \times \mathbb{P}^{2},
$$

так что построенные выше бирациональные отображения $p_{x}$ порождают рациональное доминантное отображение $p: C \times \mathbb{P}^{2} \rightarrow V$, что и требуется, так как $C \times \mathbb{P}^{2}$ бирационально эквивалентно $\mathbb{P}^{3}$. 
Напомним (принадлежащую Сегре) конструкцию унирациональной трехмерной квартики. Попытаемся действовать по образцу кубической гиперповерхности, разобранному выше. Пусть $V=V_{4} \subset \mathbb{P}^{4}$ - гладкая квартика, $x \in V$ точка общего положения. Для того чтобы пересечение $L \cap V, L \ni x$ - некоторая прямая, состояло из двух точек, необходимо, чтобы $L$ имело двойное касание с $V$ в точке $x$, т. е. $(L \cdot V)_{x}=3$. В этом случае $(L \circ V)=3 x+p(L)$, где $p(L) \in V-$ вычетная точка, и рациональное отображение $p$ корректно определено.

Рассмотрим некоторую систему аффинных координат $z_{1}, \ldots, z_{4}$ на $\mathbb{P}^{4}$ с началом в точке $x$. Квартика $V$ задается неоднородным многочленом $f\left(z_{*}\right)=$ $q_{1}+q_{2}+q_{3}+q_{4}$, где $q_{i}\left(z_{*}\right)$ - однородный многочлен степени $i$. Прямая $L \ni x$, $L=\left\{t\left(a_{1}, \ldots, a_{4}\right) \mid t \in \mathbb{C}\right\}$ имеет по крайней мере двойное касание с $V$ в точке $x$ тогда и только тогда, когда $q_{1}\left(a_{*}\right)=q_{2}\left(a_{*}\right)=0$. Интерпретируя $\left(a_{*}\right)$ как однородные координаты на проективизированном касательном пространстве $\mathbb{P}\left(T_{x} \mathbb{P}^{4}\right) \cong \mathbb{P}^{3}$, так что плоскость $\left\{q_{1}=0\right\} \subset \mathbb{P}^{3}$ есть $\mathbb{P}\left(T_{x} V\right)$, видим, что нужные нам прямые параметризуются коникой $Q(x)=\left\{q_{1}=q_{2}=0\right\} \subset \mathbb{P}\left(T_{x} V\right)$. Имеем корректно определенное отображение $p_{x}: Q(x) \rightarrow V$.

Нетрудно построить явные примеры квартик, содержащих рациональную поверхность $S \subset V$ (играющую ту же самую роль, что рациональная кривая $C$ для кубической гиперповерхности выше). Классическая конструкция: пусть $S=\left\{G\left(x_{1}, \ldots, x_{4}\right)=0\right\} \subset \mathbb{P}^{3}-$ рациональная квартика с единственной тройной точкой $(1: 0: 0: 0)$ (проекция $S \rightarrow \mathbb{P}^{2}$ из которой есть бирациональная эквивалентность), тогда общий многочлен вида $G+x_{0} H$, где $H\left(x_{0}, \ldots, x_{4}\right)$ однородный многочлен степени три, задает квартику $V \supset S$. Получаем рациональное доминантное отображение

$$
p: Q_{S}=\bigvee_{x \in S} Q(x) \rightarrow V .
$$

Многообразие $Q_{S}$, снабженное естественной проекцией $\pi: Q_{S} \rightarrow S$, есть расслоение на коники (см. п. 2.3 ниже). К сожалению, типичное расслоение на коники нерационально, так что наличие отображения $p: Q_{S} \rightarrow V$ еще не означает унирациональности $V$. Хорошо известно, однако, что расслоение на коники, обладающее сечением, рационально. Оставляем элементарное доказательство читателю в качестве упражнения: с точностью до послойной бирациональной эквивалентности многообразие $Q_{S}$ задается одним уравнением

$$
\sum_{0 \leqslant i \leqslant j \leqslant 2} A_{i j} u_{i} u_{j}=0
$$

в $S \times \mathbb{P}^{2}$, где $A_{i j}$ - некоторые рациональные функции на поверхности $S,\left(u_{0}\right.$ : $\left.u_{1}: u_{2}\right)$ - однородные координаты на $\mathbb{P}^{2}$; теперь можно применить послойную стереографическую проекцию из сечения.

Несложно так подобрать многочлены $G$ и $H$, чтобы расслоение $\pi: Q_{S} \rightarrow S$ имело сечение (а квартика $V$ была гладкой); это еще одно упражнение для читателя (примеры приведены в [3]). Для таких специальных гладких квартик описанная выше конструкция дает унирациональность. Отметим, что унирациональность общей трехмерной квартики остается открытой проблемой. 
Итак, произвольная гладкая кубика $V_{3} \subset \mathbb{P}^{4}$ и некоторые специальные гладкие квартики $V_{4} \subset \mathbb{P}^{4}$ унирациональны. Главная трудность в решении проблемы Люрота заключалась, однако, в том, чтобы доказать нерациональность этих многообразий, что и было сделано в работах Г. Клеменса и Ф. Гриффитса [8] и В.А. Исковских и Ю. И. Манина [3].

Отрицательное решение проблемы Люрота означало, что проблему рациональности нельзя решить в терминах дифференциально-геометрических инвариантов (в размерности три и выше). Более того, возникла проблема геометрической характеризации многообразий, не имеющих нетривиальных дифференциально-геометрических инвариантов. Стало ясно, что для удовлетворительной классификации многомерных алгебраических многообразий необходимо вводить новые классы многообразий, а проблема рациональности есть (важный) частный случай более общих вопросов.

Вот почему прорыв, осуществленный в работах [3], [8], имел такое большое значение.

Отметим, что каждая из работ [3], [8] породила новое направление исследований: так называемые трансцендентный метод и метод максимальных особенностей соответственно. С помощью трансцендентного метода были получены очень точные результаты о нерациональности трехмерных расслоений на коники, а техника вырождения Клеменса позволила доказать нерациональность общих (в комплексно-аналитическом смысле) пучков кубических поверхностей и многих классов трехмерных многообразий Фано [33], [34]. А. С. Тихомиров применил трансцендентный метод к решению более тонкой задачи о существовании структур расслоения на коники на некоторых многообразиях Фано [35]-[37]. Однако сфера применимости этого замечательного метода остается ограниченной классом трехмерных многообразий. В настоящем обзоре мы не касаемся метода Клеменса-Гриффитса (иногда также называемого методом промежуточного якобиана) и отсылаем читателя к обзорной статье [34].

Предметом данной статьи является метод максимальных особенностей, аккумулировавший идеи Нётера и Фано. Несколько забегая вперед, отметим, что из результатов [3] немедленно, без каких-либо дополнительных рассуждений, вытекало, что гладкая трехмерная квартика $V=V_{4} \subset \mathbb{P}^{4}$ не может быть расслоена рациональным отображением $\varphi: V \rightarrow Y$ на рациональные поверхности (случай $Y=\mathbb{P}^{1}$ ) или кривые (случай $Y=\mathbb{P}^{2}$ : в силу того, что в малых размерностях проблема Люрота решается положительно, образом рационального доминантного отображения $\varphi$ с $\operatorname{dim} Y<\operatorname{dim} V$ может быть либо рациональная кривая, либо рациональная поверхность). Поскольку линейная проекция $\mathbb{P}^{3} \rightarrow \mathbb{P}^{k}, k=1,2$, расслаивает $\mathbb{P}^{3}$ на плоскости и прямые соответственно, это дает еще одно доказательство нерациональности гладкой трехмерной квартики, причем, как вскоре выяснилось [16], это рассуждение лучше, чем апелляция к конечности группы бирациональных автоморфизмов $\operatorname{Bir} V=\operatorname{Aut} V$, потому что имеются другие многообразия, для которых последняя группа бесконечна, но нерациональность которых все же можно доказать через отсутствие структур расслоения на рациональные поверхности или кривые.

Таким образом, на первый план вышел следующий вопрос (или, скорее, класс вопросов): описать, для заданного многообразия $V$, все рациональные 
отображения $\varphi: V \rightarrow Y$, база $Y$ и слой общего положения $\varphi^{-1}(y), y \in Y$, которых принадлежат к данному классу многообразий. Прежде чем приводить точные определения, рассмотрим еще один пример - теорему В.Г. Саркисова о (многомерных) расслоениях на коники [38], [39]. Теорема Саркисова была особенно впечатляющим прорывом начала 80-х годов ввиду наглядности и простоты результата (простоты формулировки, но, конечно, не доказательства). Она еще раз показала, что сама проблема рациональности нуждается в обобщении для построения адекватной многомерной теории, и подтвердила направление, в котором такое обобщение следовало искать.

2.3. Теорема Саркисова о расслоениях на коники. Пусть $S$ - гладкое проективное многообразие размерности $\operatorname{dim} S \geqslant 2, \rho: E \rightarrow S$ - (алгебраическое) векторное расслоение ранга $3, \rho: \mathbb{P}(E) \rightarrow S$ - его проективизация, т. е. локально тривиальное $\mathbb{P}^{2}$-расслоение над $S$. Гиперповерхность $V \subset \mathbb{P}(E)$, снабженная естественной проекцией $\pi: V \rightarrow S, \pi=\left.\rho\right|_{V}$, называется расслоением на коники над $S$, если каждый слой $\pi^{-1}(s) \subset \mathbb{P}^{2}=\rho^{-1}(s)$ есть коника в $\mathbb{P}^{2}$. Если $\mathscr{E}$ - пучок сечений двойственного расслоения $E^{*} \rightarrow S$, то $\mathbb{P}(E)$ совпадает с проективным расслоением $\mathbb{P}(\mathscr{E})$ в смысле Гротендика (Proj, cм. [4]). Пусть $\mathscr{L}$ - тавтологический пучок на $\mathbb{P}(\mathscr{E})$. Гиперповерхность $V$ задается сечением линейного расслоения $\mathscr{L}^{\otimes 2} \otimes \rho^{*} \mathscr{F}$, где $\mathscr{F}$ - некоторый обратимый пучок на базе $S$. Простейший пример расслоения на коники - это дивизор бистепени $(2, N)$ на прямом произведении $\mathbb{P}^{2} \times \mathbb{P}^{M}, M \geqslant 2$. Ввиду локальной тривиальности расслоения $E$ любое расслоение на коники бирационально эквивалентно расслоению последнего примера, однако такая эквивалентность ничего не упрощает, а наоборот, все усложняет, поскольку неособому многообразию $V \subset \mathbb{P}(E)$ соответствует некоторое многообразие $V^{\sharp} \subset \mathbb{P}^{2} \times \mathbb{P}^{M}$, имеющее, вообще говоря, неконтролируемые особенности и, возможно, содержащее целые плоскости $\mathbb{P}^{2} \times\{\rho\}, \rho \in \mathbb{P}^{M}$. "Правильным" объектом изучения являются стандартные расслоения на коники $\pi: V \rightarrow S$, где $V$ - гладкое многообразие, удовлетворяющее следующему условию минимальности: $\operatorname{Pic} V=\mathbb{Z} K_{V} \oplus \pi^{*} \operatorname{Pic} S$. Из этого условия вытекает, что расслоение $V / S$ не имеет сечений (в бирациональном смысле, т. е. над открытым по Зарискому подмножеством $U \subset S)$ : любой дивизор $D$ на $V$ имеет четный индекс пересечения с общим слоем $C_{s}=\pi^{-1}(s)$, так как $\left(K_{V} \cdot C_{s}\right)=-2$ и $\left(\pi^{*} R \cdot C_{s}\right)=0$ для любого дивизора $R$ на базе $S$. Поэтому расслоение $V / S$ послойно не тривиализуемо (в бирациональном смысле!). Конструкция Сегре из п. 2.2 представляет пример противоположной ситуации.

С каждым стандартным расслоением на коники $\pi: V \rightarrow S$ связан важный объект - дискриминантный дивизор $D \subset S$, состоящий из точек $x \in S$, слой над которыми $\pi^{-1}(x) \subset \mathbb{P}^{2}$ есть негладкая коника: пара прямых (для точки общего положения $x \in D)$ или двойная прямая. Если $V \subset \mathbb{P}^{2} \times \mathbb{P}^{M}$ задается уравнением

$$
\sum_{i, j=0}^{2} A_{i j}\left(x_{*}\right) y_{i} y_{j}=0
$$

$\left(y_{0}: y_{1}: y_{2}\right)$ - однородные координаты на $\mathbb{P}^{2},\left(x_{*}\right)$ - на $\mathbb{P}^{M}, A_{i j}=A_{j i}$ - однородные многочлены степени $N \geqslant 1$, то дискриминантный дивизор $D \subset \mathbb{P}^{M}$ 
задается уравнением $\operatorname{det}\left\|A_{i j}\right\|=0$ (откуда и происходит его название). Теорема Саркисова утверждает, что бирациональная геометрия многообразия $V$ определяется свойствами пары $(S, D)$.

Теорема 1.6. Пусть $\pi: V \rightarrow S$ - стандартное расслоение на коники, удовлетворяющее условию $\left|4 K_{S}+D\right| \neq \varnothing$. Тогда на $V$ нет других структур расслоения на коники, кроме $\pi$ : если $\varepsilon: V \rightarrow S^{+}$есть рациональное доминантное отображение, общий слой $\varepsilon^{-1}(s), s \in S^{+}$, которого есть рациональная кривая, то существует бирациональное отображение $\alpha: S \rightarrow S^{+}$такое, что $\varepsilon=\alpha \pi$, m.е. диаграмма

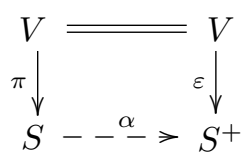

коммутативна.

Таким образом, в предположениях теоремы Саркисова многообразие $V$ может быть расслоено (рациональным отображением) на рациональные кривые единственным способом. Поскольку для проективного пространства $\mathbb{P}^{n}$ структур расслоения на коники бесконечно много (одних только линейных проекций из точки $\mathbb{P}^{n} \rightarrow \mathbb{P}^{n-1}$ имеется $n$-мерное семейство), получаем

СледСтвиЕ 1.1. В предположениях теоремъ 1.6 многообразие $V$ нерационально.

Прорыв, осуществленный в работах Саркисова [38], [39] был тем более впечатляющим, что охватывал все размерности $(\geqslant 3)$. Для сравнения: конкурирующий трансцендентный метод до сих пор ограничен рамками трехмерной геометрии. Правда, результаты о нерациональности расслоений на коники, полученные трансцендентным методом, являются более точными (охватывают несколько больший класс многообразий).

Отметим, что расслоения на коники имеют огромную группу послойных бирациональных автоморфизмов $\operatorname{Bir}(V / S)$, т. е. автоморфизмов, переводящих каждый слой в себя. Чтобы это увидеть, рассмотрим произвольную гладкую конику $C \subset \mathbb{P}^{2}$. Пусть $x \in \mathbb{P}^{2} \backslash C$ - некоторая точка, $\rho_{x}: \mathbb{P}^{2}--\mathbb{P}^{1}$ - линейная проекция из точки $x,\left.\rho_{x}\right|_{C}: C \rightarrow \mathbb{P}^{1}-$ ее ограничение на кривую $C$. Очевидно, $\left.\rho_{x}\right|_{C}$ есть двойное накрытие, так что имеется инволюция Галуа $\tau_{x} \in \operatorname{Aut} C$, переставляющая точки в слоях. Группа Aut $C=$ Aut $\mathbb{P}^{1}$ порождается этими инволюциями. Важность инволюций $\tau_{x}$ в том, что их конструкцию легко глобализовать до послойного бирационального автоморфизма $\tau_{\sigma} \in \operatorname{Bir}(V / S)$, связанного с произвольным рациональным сечением $\sigma: S \rightarrow \mathbb{P}(\mathscr{E})$ проективного расслоения $\rho: \mathbb{P}(\mathscr{E}) \rightarrow S$ (напомним, что $V$ вложено в $\mathbb{P}(\mathscr{E})$, причем $C_{s}=\pi^{-1}(s)$ вкладывается в $\mathbb{P}^{2}=\rho^{-1}(s)$ как коника). Отображение $\tau_{\sigma}$ не является, вообще говоря, регулярным по двум причинам: во-первых, сечение $\tau$ не всюду определено, а во-вторых, автоморфизм $\tau_{\sigma}$ не определен на тех слоях $C_{s}, s \in S$, которые пересекаются с образом сечения $\sigma$, т. е. $\sigma(s) \in C_{s}$. Поскольку $\mathbb{P}^{2}-$ расслоение $\mathbb{P}(\mathscr{E}) / S$ локально тривиально (в алгебраическом смысле), рациональные сечения соответствуют рациональным отображениям $S \rightarrow \rightarrow \mathbb{P}^{2}$. Это 
дает представление о "размере" группы $\operatorname{Bir}(V / S)$. Одним из следствий теоремы Саркисова является совпадение (с точностью до конечной подгруппы) групп $\operatorname{Bir} V$ и $\operatorname{Bir}(V / S)$.

Объясним теперь смысл условия Саркисова $\left|4 K_{S}+D\right| \neq \varnothing$ на наглядном примере расслоений на коники $V \subset \mathbb{P}^{2} \times \mathbb{P}^{M}$ бистепени $(2, N)$. Здесь $S=\mathbb{P}^{M}$, так что $K_{S}=-(M+1) H$, где $H-$ класс гиперплоскости в $\mathbb{P}^{M}$. Поэтому условие Саркисова принимает вид $\operatorname{deg} D \geqslant 4(M+1)$, а поскольку гиперповерхность $D \subset \mathbb{P}^{M}$ задается уравнением $\operatorname{det}\left\|A_{i j}\right\|=0$, получаем неравенство $3 N \geqslant 4(M+1)$. Таким образом, за исключением конечного класса семейств, все гладкие расслоения на коники данного типа удовлетворяют условию Саркисова, а само это условие можно интерпретировать как ограничение снизу на “закрученность" расслоения на коники по базе $\mathbb{P}^{M}$ в некотором интуитивно понятном смысле. Аналогичным образом обстоит дело и с расслоениями общего типа $V \subset \mathbb{P}(\mathscr{E})$, где $\mathscr{E}$ - произвольный локально свободный пучок над гладкой базой $S$. Таким образом, единственность структуры расслоения на коники является отнюдь не исключительным, а наоборот, типичным свойством, которым обладают в некотором смысле "почти все" расслоения на коники.

С точки зрения бирациональной классификации алгебраических многообразий важность теоремы Саркисова заключалась еще и в том, что были предъявлены примеры расслоений $\pi: V \rightarrow S$ в произвольной размерности, база $S$ и слой которых рациональны, но само многообразие $V$ нерационально. Это еще раз показало, что для удовлетворительной классификации в высших размерностях понятие рациональности должно быть каким-то образом обобщено, чтобы охватить все естественные примеры. Однако прежде чем переходить к описанию такого обобщения, полученного всего несколько лет назад, рассмотрим для полноты картины еще один класс трехмерных многообразий, бирациональная геометрия которых была изучена совсем недавно: пучки кубических поверхностей.

Пусть $E$ - (алгебраическое) векторное расслоение ранга 4 над проективной прямой $\mathbb{P}^{1}, \rho: \mathbb{P}(E) \rightarrow \mathbb{P}^{1}$ - его проективизация, которая является локально тривиальным $\mathbb{P}^{3}$-расслоением над $\mathbb{P}^{1}$. Удобнее пользоваться представлением многообразия $\mathbb{P}(E)$ в виде проективного расслоения $\mathbb{P}(\mathscr{E})$ в смысле Гротендика, где $\mathscr{E}-$ локально свободный пучок сечений двойственного расслоения $E^{*}$, $\mathscr{E}=\bigoplus_{i=0}^{3} \mathscr{O}_{\mathbb{P} 1}\left(a_{i}\right)$, причем можно считать, что $a_{0}=0 \leqslant a_{1} \leqslant a_{2} \leqslant a_{3}$. Гиперповерхность $V \subset \mathbb{P}(\mathscr{E})$, снабженная естественной проекцией $\pi=\left.\rho\right|_{V}: V \rightarrow \mathbb{P}^{1}$, есть расслоение на кубические поверхности над $\mathbb{P}^{1}$, если каждый слой $\pi^{-1}(t) \subset$ $\mathbb{P}^{3}=\rho^{-1}(t)$ есть кубическая поверхность. Гиперповерхность $V$ задается сечением обратимого пучка $\mathscr{L}^{\otimes 3} \otimes \rho^{*} \mathscr{O}_{\mathbb{P}^{1}}(l)$ для некоторого $l \in \mathbb{Z}$, где $\mathscr{L}$ - тавтологический пучок в смысле Гротендика. Как и в случае расслоений на коники, простейший (и модельный) пример дает гиперповерхность бистепени $(3, l)$ в $\mathbb{P}^{3} \times \mathbb{P}^{1}$.

Пусть $V \subset \mathbb{P}(\mathscr{E})$ - гладкое многообразие. Скажем, что $V$ удовлетворяет $K^{2}$-условию, если для любого $N \in \mathbb{Z}_{+}$класс $N K_{V}^{2}-f$ неэффективен, где $K_{V}-$ канонический класс многообразия $V, f \in A^{2} V$ - класс прямой в слое проекции $\pi$ (т. е. обычной прямой на кубической поверхности $\left.\pi^{-1}(t) \subset \mathbb{P}^{3}\right), A^{2} V-$ группа Чжоу классов кривых на $V$ по модулю численной эквивалентности. 
$K^{2}$-условие имеет более компактную запись: $K_{V}^{2} \notin \operatorname{Int} A_{+}^{2} V$, где $A_{+}^{2} V \subset A^{2} V \otimes \mathbb{R}$ есть замкнутый конус, порожденный классами эффективных кривых на $V$, так называемый псевдоэффективный конус. Группа Пикара $V$ есть

$$
\operatorname{Pic} V=\mathbb{Z} K_{V} \oplus \mathbb{Z} F=\mathbb{Z} L \oplus \mathbb{Z} F,
$$

где $F$ - класс слоя $\pi^{-1}(t), L-$ класс тавтологического дивизора. Для $V \subset \mathbb{P}^{3} \times \mathbb{P}^{1}$ это означает просто, что каждая поверхность на $V$ высекается гиперповерхностью бистепени $(a, b)$ : в терминах группы Пикара любой класс $D \in \operatorname{Pic} V$ имеет вид $D=a L+b F$. В этом модельном случае $K_{V}=-L+(l-2) F$ и нетрудно подсчитать, что класс кривой $K_{V}^{2}$ имеет бистепень $(3,-5 l+12)$ в $A^{3}\left(\mathbb{P}^{3} \times \mathbb{P}^{1}\right)$, т. е. как класс кривой в $\mathbb{P}^{3} \times \mathbb{P}^{1}$. При $l \geqslant 3$ вторая компонента отрицательна, так что $K^{2}$-условие выполнено. В общем случае нетрудно показать, что гиперповерхность $V$, заданная сечением пучка $\mathscr{L}^{\otimes 3} \otimes \rho^{*} \mathscr{O}_{\mathbb{P}^{1}}(l)$, удовлетворяет $K^{2}$-условию при $l \geqslant \lambda(\mathscr{E})=\lambda\left(a_{1}, a_{2}, a_{3}\right)$, т. е. при фиксированном пучке $\mathscr{E}$ это условие выполнено для всех пучков кубических поверхностей, за исключением конечного числа семейств.

ТЕОрема 1.7 [40]. Предположим, что расслоение $V \rightarrow \mathbb{P}^{1}$ удовлетворяет $K^{2}$-условию и задано достаточно общим сечением пучка $\mathscr{L}^{\otimes 3} \otimes \rho^{*} \mathscr{O}_{\mathbb{P}^{1}}(l)$ на $\mathbb{P}(\mathscr{E})$. Тогда на $V$ нет структур расслоения на коники, а проекция $\pi$ : $V \rightarrow \mathbb{P}^{1}$ есть единственная структура расслоения на рачиональные поверхности на $V$. В частности, многообразие $V$ нерационально. Группа бираицональных автоморфизмов многообразия $V$ совпадает с группой послойных бирачиональных автоморфизмов $\operatorname{Bir}\left(V / \mathbb{P}^{1}\right)$.

В силу сказанного выше, в некотором смысле почти все расслоения на кубические поверхности над $\mathbb{P}^{1}$ удовлетворяют предположениям теоремы 1.7 . Отметим, что все пучки кубических поверхностей унирациональны. Проекция $\pi: V \rightarrow \mathbb{P}^{1}$ имеет много сечений (точнее, для любого набора различных слоев $\pi^{-1}\left(t_{1}\right), \ldots, \pi^{-1}\left(t_{k}\right)$ и гладких точек $x_{i} \in \pi^{-1}\left(t_{i}\right)$ существует сечение $s: \mathbb{P}^{1} \rightarrow V$ такое, что $\left.s\left(t_{i}\right)=x_{i}\right)$, возьмем одно из них, $s: \mathbb{P}^{1} \rightarrow V$. В каждой точке $s(t) \in F_{t}=\pi^{-1}(t)$ рассмотрим рациональную кубическую кривую $C_{t}=F_{t} \cap T_{s(t)} F_{t}$, имеющую в $s(t)$ особенность. Теперь для каждой точки кривой $C_{t}$ воспроизведем конструкцию унирациональности для кубической гиперповерхности (п. 2.2), отображая общую касательную прямую к $F_{t}$ в третью точку ее пересечения с $F_{t}$. Это и дает конструкцию унирациональности для $V$. Таким образом, пучки кубических поверхностей дают новые контрпримеры к проблеме Люрота в размерности три.

Отметим, далее, что группа послойных автоморфизмов $\operatorname{Bir}\left(V / \mathbb{P}^{1}\right)$ очень велика, см. [41], [40]. Она порождается инволюциями, соответствующими сечениям и бисечениям проекции $\pi$, которых очень много.

Пучки кубических поверхностей хорошо иллюстрируют один из основных вариантов проблемы рациональности (п. 1.1): на $V$ существует единственная рациональная функция $f: V \rightarrow \mathbb{C}$ (с точностью до дробно-линейного преобразования $\mathbb{C}$ ), множества уровня $f^{-1}(a), a \in \mathbb{C}$, которой суть неприводимые рациональные поверхности. 
Итак, уже в размерности три рациональность базы и общего слоя не означает рациональности тотального пространства расслоения. Поэтому понятие рациональности должно быть обобщено. Такое обобщение мы рассмотрим в следующем параграфе.

\section{§ 3. Рационально связные многообразия}

3.1. Теорема Грабера-Харриса-Старра. Точный аналог рациональных кривых и поверхностей в высших размерностях - это рационально связные многообразия. Понятие рациональной связности восходит к работам Ю. И. Манина о геометрии кубических поверхностей, см. [41], а его обсуждение в трехмерной геометрии было начато В.С. Куликовым [42]. Активное изучение многомерных рационально связных многообразий началось независимо Кампаной [43] и Колларом, Мияокой и Мори [44].

ОПРедЕлЕНиЕ 1.2. Гладкое проективное многообразие $X$ называется рационально связным, если любые две точки $x_{1}, x_{2} \in X$ общего положения можно соединить неприводимой рациональной кривой, т. е. существует морфизм $f: \mathbb{P}^{1} \rightarrow X$ такой, что $f\left(t_{1}\right)=x_{1}$ и $f\left(t_{2}\right)=x_{2}$.

Очевидна рациональная связность проективного пространства (любые две точки соединяются прямой) и квадрики $Q \subset \mathbb{P}^{n}$ (любые две точки соединяются коникой $Q \cap P$, где $P=\mathbb{P}^{2} \subset \mathbb{P}^{n}$ - подходящая плоскость). Из определения очевидно также, что свойство быть рационально связным инвариантно относительно бирациональных отображений и что если $\varphi: X \rightarrow Y-$ рациональное доминантное отображение, причем $X$ рационально связно, то и $Y$ рационально связно. В самом деле, если $y_{1}, y_{2} \in Y$ - точки общего положения (в частности, лежащие в образе $\varphi)$, то, выбирая точки общего положения $x_{i} \in \varphi^{-1}\left(y_{i}\right)$, получаем пару общих точек $x_{1}, x_{2} \in X$, которые по предположению можно соединить рациональной кривой $f: \mathbb{P}^{1} \rightarrow X$, но тогда рациональная кривая $\varphi \circ f: \mathbb{P}^{1} \rightarrow Y$ соединяет точки $y_{1}$ и $y_{2}$.

Следующие свойства рационально связных многообразий менее очевидны, однако легко доказываются с помощью стандартной техники теории деформаций, см. [45; гл. IV]:

- любые $k$ точек $x_{1}, \ldots, x_{k}$ (т. е. без условия общности положения) можно соединить неприводимой рациональной кривой $f: \mathbb{P}^{1} \rightarrow X, f\left(t_{i}\right)=x_{i}$; более того, для любого набора ненулевых касательных векторов $v_{i} \in T_{x_{i}} X$ можно найти кривую $f$, имеющую в точности эти касательные векторы в точках $x_{i}$;

- многообразие $X$ рационально связно тогда и только тогда, когда на нем имеется оченъ свободная рациональная кривая $f: \mathbb{P}^{1} \rightarrow X$, т. е. подъем касательного расслоения относительно $f$ очень обилен:

$$
f^{*} T_{X} \cong \mathscr{O}_{\mathbb{P}^{1}}\left(a_{1}\right) \oplus \cdots \oplus \mathscr{O}_{\mathbb{P}^{1}}\left(a_{m}\right),
$$

$m=\operatorname{dim} X, a_{i} \geqslant 1 ;$ неформально говоря, в окрестности кривой $f\left(\mathbb{P}^{1}\right)$ много векторных полей, благодаря чему кривая является подвижной;

- для гладких многообразий свойство рациональной связности эквивалентно цепочной рационалъной связности, когда требуется лишь, чтобы для общих точек $x_{1}, x_{2} \in X$ существовала (возможно, приводимая) связная кривая 
$C=\bigcup_{i \in I} C_{i}$, соединяющая эти точки, $x_{i} \in C$, причем каждая компонента $C_{i}=f_{i}\left(\mathbb{P}^{1}\right)$ рациональна; таким образом, любые две точки можно соединить цепочкой рациональных кривых.

Следующий пример показывает, что если $f: X \rightarrow Y$ - морфизм гладких многообразий с неприводимыми слоями и слой общего положения рационально связен, то некоторые специальные (вырожденные) слои могут не быть рационально связными. В самом деле, возьмем общую (в частности, гладкую) гиперповерхность бистепени $(3,1)$ в $\mathbb{P}^{3} \times \mathbb{P}^{1}$, т. е. пучок кубических поверхностей в $\mathbb{P}^{3}$. Общая поверхность пучка рациональна, следовательно, рационально связна. Однако легко подобрать уравнение так, чтобы слой над некоторой точкой $t_{0} \in \mathbb{P}^{1}$ был конусом $S_{0} \subset \mathbb{P}^{3}$ над гладкой кубической кривой $C$ в $\mathbb{P}^{2}$. На конусе нет рациональных кривых, кроме образующих, так что $S_{0}$ не является рационально связным. Однако конус $S_{0}$, конечно, является цепочно рационально связным, так как все рациональные кривые на $S_{0}$ имеют общую точку - вершину конуса. На самом деле, это проявление общего факта [45; гл. IV]: если слой общего положения цепочно рационально связен, то и каждый слой цепочно рационально связен (в предположении, что $f: X \rightarrow Y$ - собственный равноразмерный морфизм). Аналогичные примеры легко построить в произвольной размерности. Они показывают, что в утверждении об эквивалентности рациональной связности и цепочной рациональной связности предположение о гладкости существенно.

Подсчитывая размерность множества рациональных кривых $f: \mathbb{P}^{1} \rightarrow \mathbb{P}^{M}$ степени $d \geqslant 1$, имеющих $d m+1$ точку пересечения с гладкой гиперповерхностью $V_{m} \subset \mathbb{P}^{M}$ степени $m, 1 \leqslant m \leqslant M$, нетрудно доказать рациональную связность гиперповерхностей Фано $V$ и, более общо, полных пересечений Фано $V_{m_{1} \cdots m_{k}}=$ $F_{m_{1}} \cap F_{m_{2}} \cap \cdots \cap F_{m_{k}} \subset \mathbb{P}^{M}$, где $F_{m_{i}}$ - гиперповерхность степени $m_{i}, m_{1}+\cdots+$ $m_{k} \leqslant M$. Это частный случай общего факта, см. [45].

\section{Теорема 1.8. Гладкое многообразие Фано V рационально связно.}

Напомним, что гладкое многообразие $V$ называется многообразием Фано, если антиканонический класс $-K_{V}$ обилен, т. е. некоторая его кратность задает вложение $V \subset \mathbb{P}^{N}$. Теоремы Клеменса-Гриффитса и Исковских-Манина показывают, что рационально связных многообразий больше, чем рациональных. Насколько больше, видно из теоремы Саркисова, так как верно

ПреДЛОЖЕНИЕ 1.3. Расслоение на коники $\pi: V \rightarrow S$ над ращионально связной базой $S$ рачионально связно.

ДокАЗАтельство. Пусть $x, y \in V$ - общая пара точек, $\bar{x}=\pi(x), \bar{y}=\pi(y)-$ ее образ на $S$. По предположению, точки $\bar{x}, \bar{y}$ лежат на неприводимой рациональной кривой $C \subset S$. Поверхность $\pi^{-1}(C)=Q$ рациональна, следовательно, рационально связна, и содержит $x, y$, что и требовалось доказать.

Таким образом, “большинство" расслоений на коники - это нерациональные рационально связные многообразия. Легко показать, что на рационально связных многообразиях нет ненулевых глобальных дифференциальных форм и, более общо, ковариантных тензоров. Поэтому класс рационально связных 
многообразий является искомым обобщением класса рациональных многообразий, но при одном условии: если этот класс замкнут относительно операции образования расслоений, т. е. для каждого расслоения $\pi: V \rightarrow S$, общий слой и база которого рационально связны, многообразие $V$ также рационально связно. Этот факт был сформулирован в качестве гипотезы в [44], однако доказан лишь десять лет спустя в [46]. Рассуждая как в доказательстве предложения 1.3 , сводим утверждение к случаю $S=\mathbb{P}^{1}$, а техника теории деформации сводит его к следующему факту, доказанному Грабером, Харрисом и Старром.

ТеОрема 1.9. Пусть $\pi: V \rightarrow \mathbb{P}^{1}$ - морфизм, общий слой которого неприводим и рачионально связен. Тогда проекиия $\pi$ имеет сечение $s: \mathbb{P}^{1} \rightarrow V$.

На самом деле, в [46] доказано существование сечения для расслоения $\pi$ : $V \rightarrow B$, где $B$ - произвольная, не обязательно рациональная, кривая. Однако случай $B=\mathbb{P}^{1}$ является основой доказательства. Не имея возможности привести здесь полное доказательство теоремы 1.9 (предметом настоящего обзора являются совсем другие геометрические идеи), прокомментируем кратко этот фундаментальный факт.

В слоях проекции $\pi$ по предположению много рациональных кривых. Если $C \subset V$ - некоторая кривая, то для точек общего положения $x_{i} \in C$, $i=1, \ldots, k, \pi\left(x_{i}\right)=t_{i} \in \mathbb{P}^{1}$, можно рассмотреть подвижные рациональные кривые $C_{i} \subset \pi^{-1}\left(t_{i}\right)$ в соответствующих слоях проекции $\pi$, проходящие через точки $x_{i}, x_{i} \in C_{i}$, и построить гребень $\Gamma=C \cup \bigcup_{i=1}^{k} C_{i}-$ связную приводимую кривую на $V$. Несложная техника теории деформаций [45] показывает, что из подвижности "прикрепленных" рациональных кривых $C_{i}$ следует подвижность кривой $\Gamma$, т. е. $\Gamma$ включается в семейство кривых $\left(\Gamma_{\lambda}, \lambda \in \Lambda\right)$, общая кривая которого неприводима. (В частности, именно поэтому, если имеется хотя бы одно сечение проекции $\pi$, то $V$ заметается сечениями.) Если кривая $C$ есть мультисечение (т. е. $\operatorname{deg}\left(\left.\pi\right|_{C}\right)=d \geqslant 2$ ), то и кривые $\Gamma_{\lambda}$ суть мультисечения той же степени $d \geqslant 2$. Основная идея доказательства Грабера-Харриса-Старра состоит в том, чтобы сравнить деформации морфизма $\mu: C \rightarrow V$ (т.е., грубо говоря, деформации кривой $C$ внутри $V$ ) и деформации конечного накрытия $\pi \circ \mu: C \rightarrow \mathbb{P}^{1}$. Если деформации $\mu$ покрывают деформации $\pi \circ \mu$, то отсюда немедленно вытекает утверждение теоремы, поскольку, согласно классической теореме Гурвица и Клебша, грубое пространство модулей $\bar{M}_{g, 0}\left(\mathbb{P}^{1}, d\right)$ имеет одну неприводимую компоненту, так что кривая $C$ может быть продеформирована внутри $V$ в приводимую кривую, распадающуюся на $d$ сечений морфизма $\pi$. Таким образом, ключевым фактом является доминантность отображения грубых пространств модулей

$$
\bar{M}_{g, 0}(V,[C]) \rightarrow \bar{M}_{g, 0}\left(\mathbb{P}^{1}, d\right),
$$

где $[C]-$ класс кривой $C \subset V$. Этот факт и доказан в [46] весьма тонкими рассуждениями, использующими геометрию гребней $\Gamma$, описанных выше.

Прямым следствием теоремы 1.9 является существование сечения $s: \mathbb{P}^{1} \rightarrow V$, пересекающего неособые слои $F_{t_{1}}, \ldots, F_{t_{k}}, F_{t_{i}}=\pi^{-1}\left(t_{i}\right)$, в произвольных наперед заданных точках $x_{i} \in F_{t_{i}}$. Это утверждение может быть усилено следующим образом [47]: для любых точек $x_{i} \in F_{t_{i}}$ на неособых слоях $F_{t_{i}}$ и любого 
набора $l_{i}$-струй неособых кривых, трансверсальных слоям $F_{t_{i}}$ в этих точках, существует сечение $s: \mathbb{P}^{1} \rightarrow V$, имеющее эти струи в точках $x_{i},\left(l_{1}, \ldots, l_{k}\right) \in \mathbb{Z}_{+}^{k}-$ произвольный набор неотрицательных целых чисел. Иными словами, можно предписывать сечению касательные векторы (трансверсальные к слоям) и, более общо, струи конечного порядка в заданных точках.

Теорема 1.9 есть естественное обобщение классической теоремы Тзена (см. [7], [45]) о существовании сечения проекции $\pi: V \rightarrow \mathbb{P}^{1}$, где $V \subset \mathbb{P}^{m} \times \mathbb{P}^{1}$ есть гиперповерхность бистепени $(d, N), d \leqslant m$. (Доказательство теоремы Тзена совершенно элементарное: сечение $s: \mathbb{P}^{1} \rightarrow \mathbb{P}^{m} \times \mathbb{P}^{1}$ представляется произвольным набором многочленов $\left(f_{0}, f_{1}, \ldots, f_{m}\right)$ на $\mathbb{P}^{1}$, условие $s\left(\mathbb{P}^{1}\right) \subset V$ дает полиномиальные уравнения для коэффициентов многочленов $f_{i}$. Число уравнений меньше числа коэффициентов, откуда все и следует.) Нетривиальное обобщение теоремы Тзена для расслоений на полные пересечения Фано в произведениях взвешенных проективных пространств дано в [48]. Поскольку полные пересечения Фано рационально связны, последний результат также является следствием теоремы Грабера-Харриса-Старра.

Теперь можно точно сформулировать задачи бирациональной геометрии, которым посвящен настоящий обзор.

3.2. Рационально связные расслоения и расслоения Фано. Сюръективный морфизм $\pi: V \rightarrow S$ проективных многообразий называется рационально связным расслоением, если база $S$ и слой общего положения $\pi^{-1}(s), s \in S$, суть рационально связные многообразия. В частности, само многообразие $V$ по теореме 1.9 рационально связно. Мы исключаем из рассмотрения тривиальные случаи $V=S$ и $S=*$ - точка. Чтобы это подчеркнуть, иногда говорим о нетривиальных расслоениях.

Структуру рационально связного расслоения на (рационально связном) многообразии $V$ можно определить двумя эквивалентными способами:

- как бирациональное отображение $\chi: V \rightarrow V^{+}$на многообразие $V^{+}$, на котором зафиксирован морфизм $\pi^{+}: V^{+} \rightarrow S^{+}$, являющийся рационально связным расслоением;

- как рациональное доминантное отображение $\varphi: V \rightarrow S$, слой общего положения которого рационально связен (и, в частности, неприводим).

Эти определения, конечно, эквивалентны в силу теоремы о разрешении особенностей [10], [11]. Структуры $\varphi_{1}: V \rightarrow S_{1}$ и $\varphi_{2}: V \rightarrow S_{2}$ считаются идентичными, если существует такое бирациональное отображение $\alpha: S_{1} \rightarrow S_{2}$, что коммутирует диаграмма рациональных отображений

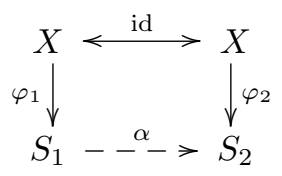

т. е. $\varphi_{2}=\alpha \circ \varphi_{1}$. Другими словами, $\varphi_{1}$ и $\varphi_{2}$ имеют те же самые слои. Множество нетривиальных структур рационально связного расслоения на многообразии $X$ (по модулю описанного отождествления) обозначается символом $R C(X)$.

Изучение структур рационально связного расслоения имеет первостепенное значение в бирациональной геометрии по следующей понятной причине. Один 
из важнейших приемов классификации алгебраических многообразий - редукция к меньшей размерности. Если алгебраическое многообразие $V$ допускает морфизм $\pi: V \rightarrow S$ на многообразие $S$ положительной размерности, где $\operatorname{dim} S<\operatorname{dim} V$, то $V$ естественно рассматривать как семейство многообразий размерности $\operatorname{dim} V-\operatorname{dim} S$ (слоев $F_{s}=\pi^{-1}(s), s \in S$ ), параметризованных базой $S$. Тем самым, описание многообразия $V$ сводится к описанию геометрии слоя общего положения $F_{s}$ и к характеризации “закрученности" семейства $F_{s}$, $s \in S$, по базе $S$.

Однако расслоения $\pi: V \rightarrow S$ можно рассматривать и с другой точки зрения. Фиксируя класс $\mathscr{S}$ многообразий $S$ и класс $\mathscr{F}$ многообразий $F_{s}$, можно строить новые многообразия размерности $\operatorname{dim} S+\operatorname{dim} F$ как расслоения с заданной базой $S \in \mathscr{S}$ и слоем $F_{s} \in \mathscr{F}$. Это дает источник построения новых алгебраических многообразий произвольной размерности.

Поэтому ясно, что исключительно важную роль в бирациональной геометрии играют рационально связные многообразия, не имеющие структур (нетривиального) рационально связного расслоения: эти многообразия "не приходят из меньших размерностей" и потому представляют собой новый классификационный тип. Особый интерес представляют и многообразия, имеющие только одну нетривиальную структуру или, по крайней мере, конечное число таких структур: для них редукция размерности определена однозначно или осуществима лишь конечным числом способов.

На множестве $R C(V)$ имеется естественное отношение частичного порядка: для $\varphi_{1}, \varphi_{2} \in R C(V)$ имеем $\varphi_{1} \leqslant \varphi_{2}$, если существует рациональное доминантное отображение $\alpha: S_{1} \rightarrow S_{2}$ такое, что диаграмма (5) коммутативна. Другими словами, слои $\varphi_{1}$ содержатся в слоях $\varphi_{2}$. Для общей точки $s \in S_{2}$ получаем

$$
\alpha^{-1}(s)=\varphi_{1}\left(\varphi_{2}^{-1}(s)\right),
$$

следовательно, $\alpha \in R C\left(S_{1}\right)$ есть структура рационально связного расслоения на $S_{1}$. Легко видеть, что соответствие $\varphi_{2} \mapsto \alpha$ определяет биекцию множеств $\left\{\psi \in R C(V) \mid \psi \geqslant \varphi_{1}\right\}$ и $R C\left(S_{1}\right)$. Поэтому с геометрической точки зрения особенный интерес представляют минималъные элементы упорядоченного множества $R C(V)$. Обозначим множество минимальных элементов символом $R C_{\min }(V)$. Пусть $R C_{d}(V) \subset R C(V)$ - множество структур, общий слой которых имеет размерность $d$. Очевидно, что если $d=\min \left\{e \in \mathbb{Z}_{+} \mid R C_{e} \neq \varnothing\right\}$, то $R C_{d} \subset R C_{\text {min }}$

Для каждого $d \in\{1, \ldots, \operatorname{dim} V-1\}$ на множестве $R C_{d}(V)$ имеется важнейшее отношение послойной бирациональной эквивалентности: $\varphi_{1} \sim \varphi_{2}$, если существуют бирациональное преобразование $\chi \in \operatorname{Bir} V$ и бирациональное отображение $\alpha: S_{1} \rightarrow S_{2}$ такие, что диаграмма

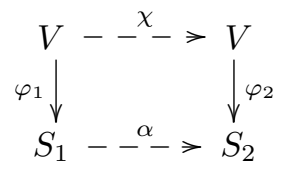

коммутативна, т. е. $\varphi_{2} \circ \chi=\alpha \circ \varphi_{1}$. Другими словами, бирациональный автоморфизм $\chi$ переводит слои $\varphi_{1}$ в слои $\varphi_{2}$. Фактормножество $R C_{d}(V) / \sim$ обозначаем символом $\overline{R C}_{d}(V)$. 
Для иллюстрации введенных понятий и конструкций рассмотрим пример, принадлежащий ученику автора И. В. Соболеву [49]. Пусть $V \subset \mathbb{P}^{3} \times \mathbb{P}^{1}-$ общий (в частности, гладкий) дивизор бистепени $(3,2), \pi: V \rightarrow \mathbb{P}^{1}-$ проекция. Очевидно, что $V / \mathbb{P}^{1}$ есть рационально связное расслоение. Как упоминалось выше, группа $\operatorname{Bir}\left(V / \mathbb{P}^{1}\right)$ послойных бирациональных автоморфизмов очень велика [41], [40]. Далее, проекция $q: V \rightarrow \mathbb{P}^{3}$ вне объединения 27 гладких рациональных кривых на $V$, стягиваемых $q$ в 27 точек на $\mathbb{P}^{3}$, имеет степень 2 , так что имеется инволюция Галуа $\tau \in \operatorname{Bir} V$, переставляющая точки в слое общего положения морфизма $q$. Методами работы [40] в [49] доказана

Tеорема 1.10. (i) На $V$ нет структур расслоения на коники: $R C_{1}(V)=\varnothing$.

(ii) Любой пучок рациональных поверхностей на $V$ может быть преобразован отображением $\chi \in \operatorname{Bir} V$ в пучок слоев проекиии $\pi: \overline{R C}_{2}(V)=\{\pi\}$.

(iii) Группа бирачиональных автоморфизмов есть свободное произведение $\operatorname{Bir} V=\operatorname{Bir}\left(V / \mathbb{P}^{1}\right) *\langle\tau\rangle$.

Таким образом, по модулю послойной бирациональной эквивалентности на $V$ имеется единственная рационально связная структура - проекция $\pi$. (Отсюда, конечно, следует нерациональность многообразия $V$.)

Множество рационально связных структур на $V$ со всеми введенными на нем структурами является бирациональным инвариантом многообразия. Поэтому для его описания (как и для решения других задач бирациональной геометрии) естественно выбрать наиболее удобную модель в классе многообразий, бирационально эквивалентных $V$ (или одну из них, если таких много). Нахождение модели с хорошими свойствами является предметом программы минимальных моделей. Законченная в размерности три, эта программа сейчас успешно продвигается в высшие размерности, см. [28], [29]. Одним из ее результатов (доказанным в трехмерном и почти доказанным в четырехмерном случае) должна стать теорема о том, что всякое рационально связное многообразие $V$ бирационально эквивалентно многообразию $V^{+}$, расслоенному проекцией $\pi^{+}: V^{+} \rightarrow S^{+}$на многообразия Фано, причем $V^{+}$имеет особенности заданного ограниченного типа и относительное число Пикара единица. Если $\operatorname{dim} V=3$ и $V^{+}$имеет $\mathbb{Q}$-факториальные терминальные особенности, то расслоение $\pi^{+}$называется расслоением Мори и гипотетическая теорема, сформулированная выше, есть (принадлежащий Мори) доказанный факт. К сожалению, уже в размерности три типов особенностей и соответствующих семейств многообразий Фано очень много - настолько много, что в настоящее время для их перечисления пишутся компьютерные программы. В данном обзоре мы будем, в основном, обсуждать гладкие многообразия Фано $V$ с условием $\operatorname{Pic} V=\mathbb{Z} K_{V}$ - так называемые примитивные многообразия Фано (типичный пример - гладкая квартика $V_{4} \subset \mathbb{P}^{4}$, с которой и началось данное направление бирациональной геометрии). По ходу дела будем комментировать известные результаты о многообразиях Фано с особенностями. В следующем обзоре, посвященном расслоениям над нетривиальной базой, будут рассмотрены стандартные расслоения Фано $\pi: V \rightarrow S$, где $V, S$ - гладкие проективные многообразия и $\operatorname{Pic} V=\mathbb{Z} K_{V} \oplus \pi^{*} \operatorname{Pic} S$. Отметим, что, несмотря на естественные 
трудности работы с особенностями, наибольшую трудность для изучения представляют именно примитивные многообразия Фано и стандартные расслоения Фано.

Как указывалось выше, особенный интерес представляют примитивные многообразия Фано $V$, не имеющие нетривиальных структур рационально связного расслоения, $R C(V)=\varnothing$. Эти многообразия являются основным предметом настоящего обзора. Мы покажем, что они удовлетворяют условию бирациональной (сверх)жесткости, которая позволяет дать полное описание их бирациональной геометрии (вычислить $R C(V)$, образующие и соотношения группы бирациональных автоморфизмов $\operatorname{Bir} V$ и многое другое). Отметим, что некоторые авторы [2], [50]-[57] принимают отсутствие нетривиальных рационально связных структур за определение бирациональной жесткости, в то время как мы пользуемся традиционным, менее наглядным и более техничным, но в конечном итоге более фундаментальным определением бирациональной жесткости (см. п. 2.1 главы 2 ниже).

Для полноты картины добавим, что для стандартных расслоений Фано $\pi$ : $V \rightarrow S$ над нетривиальной фиксированной базой $S$, слои $F_{s}=\pi^{-1}(s), s \in S$, которых принадлежат заданному семейству $\mathscr{F}$ многообразий Фано, бирациональная геометрия определяется следующим неформальным принципом:

при "достаточной закрученности" расслоения $\pi$ по базе $S$ бирациональная геометрия многообразия $V$ сводится $к$ бирациональной геометрии слоя, структура $\pi$ является наименъшим элементом упорядоченного множества $R C(V)$, а бирациональные автоморфизмы многообразия $V$ являются послойными.

Теперь, определив точно класс объектов и набор вопросов, которые мы будем задавать, опишем кратко структуру основной (технической) части данной работы.

3.3. Метод максимальных особенностей. Суть метода, являющегося сегодня основным инструментом изучения бирациональных отображений многообразий Фано и расслоений Фано, заключается в следующем. На рационально связном многообразии нет глобальных дифференциальных форм, однако можно сказать гораздо больше: на нем выполнено классическое условие обрыва присоединения канонического класса: для любого дивизора $D$ линейная система $\left|D+N K_{V}\right|$ пуста при $N \gg 0$, потому что канонический класс отрицателен на всяком семействе рациональных кривых, заметающих $V$. Начиная с работ итальянской школы было осознано, что важнейшую роль играет параметр, измеряющий момент обрыва присоединения, в современной терминологии - порог канонического присоединения (подробно обсуждаемый ниже). Для изучения бирационального отображения

$$
\chi: V \rightarrow V^{\prime}
$$

необходимо сравнить пороги канонического присоединения произвольного эффективного дивизора $D^{\prime}$ на $V^{\prime}$ и его собственного прообраза $D$ на $V$. Если присоединение канонического класса на $V$ обрывается не позже, чем на $V^{\prime}$ для 
любого подвижного дивизора $D^{\prime}$, то оказывается, что отображение $\chi$ допускает (сравнительно) простое описание. Поэтому вся работа происходит с отображениями, не удовлетворяющими этому предположению, так что на $V, V^{\prime}$ имеются линейные системы $\Sigma, \Sigma^{\prime}$ без неподвижных компонент такие, что $\Sigma$ есть собственный прообраз $\Sigma^{\prime}$ и каноническое присоединение для $\Sigma^{\prime}$ обрывается раньше, чем для $\Sigma$. Это - исходная точка работы; основным объектом является подвижная система $\Sigma$, а о системе $\Sigma^{\prime}$ можно забыть. Теперь необходимо сделать следующие шаги.

I. Доказать существование подмножеств большой кратности в базисном множестве системы $\Sigma$, так называемых максимальных особенностей (давших название методу); соответствующее условие "иметь большу́ю кратность" обобщает неравенство Нётера для $\mathbb{P}^{2}$ (п. 1.3) и неравенство Фано (п. 2.1) и потому называется неравенством Нётера-Фано.

II. Определить, какие максимальные особенности могут реализоваться на данном многообразии $V$ и какие нет; эта процедура называется исключением максимальных особенностей и является самой трудной частью работы.

III. Для потенциально реализуемых максимальных особенностей (выделенных на предыдущем этапе) построить “откручивающие” бирациональные отображения, упрощающие отображение $\chi$ (или линейную систему $\Sigma$ ); эта процедура называется откручиванием максимальных особенностей и обычно не представляет больших трудностей, хотя иногда может быть весьма нетривиальной.

С чисто практической точки зрения главный недостаток метода максимальных особенностей состоит в том, что если хотя бы один тип максимальной особенности не откручен и не исключен (т. е. мы не можем ни показать, что данный тип не реализуется, ни предъявить откручивающего отображения), то независимо от объема проделанной работы почти ничего нельзя сказать о бирациональной геометрии данного многообразия, а вся полученная информация ни с какой точки зрения не является законченным результатом. Это обстоятельство в какой-то мере компенсируется тем, что если удается исключить или открутить все типы максимальных особенностей, то получается исчерпывающее описание бирациональных отображений для данного многообразия.

Этап I на современном уровне был реализован в знаменитой работе В. А. Исковских и Ю. И. Манина о трехмерной квартике [3]. По сути, именно в [3] были открыты канонические особенности пар (см. п. 1.2 главы 2). Фано мог объяснить свою идею максимальной особенности, лишь приводя примеры; в [3] был предложен компактный формализм, позволяющий дать точное определение, с которым можно работать.

Этап III геометрически наиболее наглядный. Максимальные особенности успешно откручивали и Нётер, и Кастельнуово, и Фано - последний продвинулся в этом направлении очень далеко [25]. При условии, что этап II успешно реализован, откручивание представляет собой, можно сказать, наиболее приятную часть работы - несложную, но результативную.

Почти всю сложность аккумулирует этап II. Стоит отметить, что в пионерской работе В. А. Исковских и Ю. И. Манина этап III вообще отсутствует: 
на трехмерной квартике откручивать нечего, ни один тип максимальной особенности не может реализоваться. Значение работы [3] в том, что в ней впервые был предложен эффективный метод исключения максимальных особенностей. Разработанная в [3] техника весьма сложная, но ее главный недостаток был в том, что она позволяла исключать максимальные особенности лишь на гладких многообразиях малой (не более 4) степени. Первые попытки применить этот метод к многообразиям более высокой степени (полное пересечение $V_{2 \cdot 3} \subset \mathbb{P}^{5}$ квадрики и кубики [16]) и особым многообразиям (трехмерная квартика с двойной точкой) не увенчались успехом. Вместе с тем, с геометрической точки зрения многообразия большей степени интереснее; гладких многообразий Фано малой степени вообще очень мало. Долгое время казалось, что предложенный в [3] метод позволяет получать лишь изолированные результаты исключительного типа [16], а большинство многообразий Фано (тем более - в высших размерностях) недоступно для изучения на этом пути. Такие представления (в основе которых - неудачи 70-80-х годов) держались вплоть до 1996 г. (см. введение к [58]), когда положение начало меняться. (Работы [59]-[61], сделанные в духе классической техники, хорошо показывают, как экспоненциально возрастали трудности при небольшом увеличении степени или допущении хотя бы одной невырожденной двойной точки.)

В [62], [63] техника пробного класса, составляющая основу работы [3], была существенно переосмыслена и упрощена (статья [63], опубликованная в 2000 г., была написана и стала доступной всем работающим в данной области в 1996 г.). Этот новый подход в сочетании с рядом новых идей позволил эффективно работать с трехмерными многообразиями, расслоенными на поверхности дель Пеццо над $\mathbb{P}^{1}$, и некоторыми расслоениями Фано над $\mathbb{P}^{1}$ [40] (стоит отметить, что до появления последней работы не было ни одного результата в этом направлении; более подробно о расслоениях над нетривиальной базой будет рассказано в последующей обзорной статье). Наконец, в [64] была развита техника гиперкасательных дивизоров, позволившая применить метод к многообразиям Фано произвольно высокой степени и размерности и к большим классам расслоений Фано над $\mathbb{P}^{1}[65]-[76]$, а также к прямым произведениям Фано [77]. Метод максимальных особенностей стал основным инструментом изучения бирациональной геометрии многообразий и расслоений Фано.

В настоящем обзоре дается последовательное введение в это направление в его современном виде. Опишем кратко структуру основной части статьи. В главе 2 сосредоточена “классическая теория": условие обрыва присоединения канонического класса и пороги канонического присоединения, измеряющие скорость обрыва; определение бирациональной (сверх)жесткости и ее главные геометрические последствия; определение и примеры максимальных особенностей; общая схема метода максимальных особенностей; простейшие примеры исключения максимальных особенностей и $4 n^{2}$-неравенство; описание процедуры откручивания максимальных особенностей (на примере полного пересечения $\left.V_{2 \cdot 3} \subset \mathbb{P}^{5}\right)$.

Глава 3 посвящена "индустриальным" методам, позволяющим охватывать сразу большие классы многообразий, прежде всего, технике гиперкасательных дивизоров. Эта техника работает для многообразий общего положения, 
удовлетворяющих специальным условиям регулярности (однако многообразие общего положения может иметь особенности, удовлетворяющие, в свою очередь, условиям регулярности). Для того чтобы полученные таким образом результаты были осмысленными, необходимо для каждого набора условий регулярности доказывать, что многообразие общего положения этим условиям удовлетворяет; такая проверка нетривиальна и требует специальной работы. Методы проверки условий регулярности будут приведены.

Основным результатом, полное доказательство которого будет приведено в данной работе, является доказательство бирациональной сверхжесткости (в частности, отсутствие нетривиальных структур рационально связного расслоения, совпадение групп бирациональных и бирегулярных автоморфизмов и, как очень частное следствие, нерациональность) общих полных пересечений Фано $V_{d_{1} \cdots d_{k}}=F_{1} \cap \cdots \cap F_{k} \subset \mathbb{P}^{N}, \operatorname{deg} F_{i}=d_{i}, d_{1}+\cdots+d_{k}=N$, произвольной размерности (с ограничением $k<(1 / 2) \operatorname{dim} V$ на число уравнений) и некоторых других естественных классов многообразий Фано.

\section{Глава 2. Метод максимальных особенностей}

\section{§1. Обрыв канонического присоединения}

1.1. Порог канонического присоединения и бирациональная жесткость. Гладкое проективное рационально связное многообразие $X$ удовлетворяет классическому условию обрыва присоединения канонического класса: для любого эффективного дивизора $D$ линейная система $\left|D+m K_{X}\right|$ пуста при $m \gg 0$, поскольку $K_{X}$ отрицателен на любом семействе рациональных кривых, заметающих $X$, а эффективный дивизор неотрицателен на любом таком семействе. Как отмечалось выше, важно точно зафиксировать момент обрыва. Чтобы формализовать эту идею, рассмотрим группу Пикара $A^{1} X=\operatorname{Pic} X$, положим $A_{\mathbb{R}}^{1} X=A^{1} X \otimes \mathbb{R}$ и определим конусы $A_{+}^{1} X \subset A_{\mathbb{R}}^{1} X$ псевдоэфбективньи классов и $A_{\mathrm{mov}}^{1} X \subset A_{\mathbb{R}}^{1} X$ nодвижных классов как замкнутые (в обычной вещественной топологии $A_{\mathbb{R}}^{1} X \cong \mathbb{R}^{k}$ ) конусы, порожденные классами эффективных дивизоров и подвижных дивизоров (т.е. дивизоров в линейных системах без неподвижных компонент) соответственно.

ОПРЕДЕЛЕНИЕ 2.1. Порог Канонического присоединения дивизора $D$ на многообразии $X$ есть число $c(D, X)=\sup \left\{\varepsilon \in \mathbb{Q}_{+} \mid D+\varepsilon K_{X} \in A_{+}^{1} X\right\}$. Если $\Sigma-$ непустая линейная система на $X$, полагаем $c(\Sigma, X)=c(D, X)$, где $D \in \Sigma-$ произвольный дивизор.

Пример 2.1. (i) Пусть $X$ - примитивное многообразие Фано, т.е. гладкое проективное многообразие с обильным антиканоническим классом и $\operatorname{Pic} X=\mathbb{Z} K_{X}$. Для любого эффективного дивизора $D$ имеем $D \in\left|-n K_{X}\right|$ для некоторого $n \geqslant 1$, так что $c(D, X)=n$. Если заменить условие $\operatorname{Pic} X=\mathbb{Z} K_{X}$ более слабым условием $\operatorname{rk} \operatorname{Pic} X=1$, т. е. $K_{X}=-r H$, где $\operatorname{Pic} X=\mathbb{Z} H, r \geqslant 2-$ индекс многообразия $X$, то для $D \in|n H|$ получаем $c(D, X)=n / r$.

Это тривиальный пример, поскольку пространство $A_{\mathbb{R}}^{1} X \cong \mathbb{R}$ одномерно и $A_{+}^{1} X=\mathbb{R}_{+}$есть положительный луч, $K_{X} \in \mathbb{R}_{-}$. 
(ii) Пусть $\pi: V \rightarrow S$ - рационально связное расслоение с $\operatorname{dim} V>\operatorname{dim} S \geqslant 1$, $\Delta-$ эффективный дивизор на базе $S$. Очевидно, что $c\left(\pi^{*} \Delta, V\right)=0$. Если Pic $V=\mathbb{Z} K_{V} \oplus \pi^{*}$ Pic $S$, т. е. $V / S$ - стандартное расслоение Фано, и $D$ - эффективный дивизор на $V$, который не поднят с базы $S$, то $D \in\left|-n K_{V}+\pi^{*} R\right|$ для некоторого дивизора $R$ на $S$, где $n \geqslant 1$. Очевидно, что $c(D, V) \leqslant n$ и, более того, если дивизор $R$ эффективен, то $c(D, V)=n$.

В самом деле, $K_{V}$ отрицателен на слоях морфизма $\pi$ (в частности, на плотных семействах рациональных кривых, заметающих слои проекции $\pi$ ), в то время как любой дивизор, поднятый с базы, тривиален на слоях.

(iii) Пусть $F_{1}, \ldots, F_{K}$ - примитивные многообразия Фано, $V=F_{1} \times \cdots \times F_{K}-$ их прямое произведение. Пусть $H_{i}=-K_{F_{i}}$ - положительная образующая группы Pic $F_{i}$. Положим

$$
S_{i}=\prod_{j \neq i} F_{j},
$$

так что $V \cong F_{i} \times S_{i}$. Пусть $\rho_{i}: V \rightarrow F_{i}$ и $\pi_{i}: V \rightarrow S_{i}$ - проекции на сомножители. Несколько злоупотребляя обозначениями, мы пишем $H_{i}$ вместо $\rho_{i}^{*} H_{i}$, так что

$$
\text { Pic } V=\bigoplus_{i=1}^{K} \mathbb{Z} H_{i}
$$

и $K_{V}=-H_{1}-\cdots-H_{K}$. Для любого эффективного дивизора $D$ на $V$ получаем

$$
D \in\left|n_{1} H_{1}+\cdots+n_{K} H_{K}\right|
$$

для некоторых неотрицательных $n_{1}, \ldots, n_{K} \in \mathbb{Z}_{+}$и, очевидно,

$$
c(D, V)=\min \left\{n_{1}, \ldots, n_{K}\right\} .
$$

Этот пример сводится к предыдущему: предположим, что $c(D, V)=n_{1}$, и положим $n=n_{1}, \pi=\pi_{1}, F=F_{1}, S=S_{1}$. Получаем

$$
\Sigma \subset\left|-n K_{V}+\pi^{*} Y\right|
$$

где $Y=\sum_{i=2}^{K}\left(n_{i}-n\right) H_{i}$ - эффективный класс на базе $S$ расслоения $\pi: V \rightarrow S$. Это случай примера (ii) выше.

Порог канонического присоединения легко вычисляется, однако главный недостаток этого понятия - его бирациональная неинвариантность.

Пример 2.2. Пусть $\pi: \mathbb{P}^{M} \rightarrow \mathbb{P}^{m}$ - линейная проекция из $(M-m-1)$-мерного подпространства $P \subset \mathbb{P}^{M}$. Рассмотрим подвижную линейную систему $\Lambda$ гиперповерхностей степени $n$ в $\mathbb{P}^{m}$ и пусть $\Sigma$ - ее прообраз относительно $\pi$. Очевидно, что $c\left(\Sigma, \mathbb{P}^{M}\right)=n /(M+1)$. Раздуем, однако, плоскость $P$, скажем, $\sigma: \mathbb{P}^{+} \rightarrow \mathbb{P}^{M}$, так что композиция $\pi \circ \sigma: \mathbb{P}^{+} \rightarrow \mathbb{P}^{m}$ есть $\mathbb{P}^{M-m}$-расслоение. Пусть $\Sigma^{+}$- собственный прообраз системы $\Sigma$ на $\mathbb{P}^{+}$. Поскольку $\pi \circ \sigma-$ морфизм с рационально связными слоями, получаем $c\left(\Sigma^{+}, \mathbb{P}^{+}\right)=0$. Этот пример легко обобщить на линейные проекции полных пересечений Фано $V \subset \mathbb{P}^{M}$ индекса 2 или выше. 
Чтобы преодолеть бирациональную неинвариантность порога канонического присоединения, дадим следующее определение.

ОПРЕДЕЛЕНИЕ 2.2. Для подвижной линейной системы $\Sigma$ на многообразии $X$ определим виртуалъный порог канонического присоединения формулой

$$
c_{\mathrm{virt}}(\Sigma)=\inf _{X^{\sharp} \rightarrow X}\left\{c\left(\Sigma^{\sharp}, X^{\sharp}\right)\right\},
$$

где точная нижняя грань берется по всем бирациональным морфизмам $X^{\sharp} \rightarrow X$, $X^{\sharp}$ - гладкая проективная модель $\mathbb{C}(X), \Sigma^{\sharp}-$ собственный прообраз системы $\Sigma$ на $X^{\sharp}$.

Виртуальный порог очевидным образом есть бирациональный инвариант пары $(X, \Sigma)$ : если $\chi: X \rightarrow X^{+}-$бирациональное отображение, $\Sigma^{+}=\chi_{*} \Sigma-$ собственный прообраз системы $\Sigma$ относительно $\chi^{-1}$, то получаем $c_{\text {virt }}(\Sigma)=$ $c_{\text {virt }}\left(\Sigma^{+}\right)$.

ПРЕДЛОЖЕНИЕ 2.1. (i) Предположим, что на многообразии V нет подвижных линейных систем с нулевым виртуальным порогом канонического присоединения. Тогда на $V$ нет структур нетривиального расслоения на многообразия отрищательной кодаировой размерности, т.е. не существует раицонального доминантного отображения $\rho: V \rightarrow S, \operatorname{dim} S \geqslant 1$, общий слой которого имеет отрищательную кодаирову размерность.

(ii) Пусть $\pi: V \rightarrow S$ - рачионально связное расслоение. Предположим, что каждая подвижная линейная система $\Sigma$ на $V$ с нулевым виртуальным порогом канонического присоединения, $c_{\text {virt }}(\Sigma)=0$, есть прообраз системы на базе: $\Sigma=\pi^{*} \Lambda$, где $\Lambda$ - некоторая подвижная линейная система на S. Тогда любое бирачиональное отображение

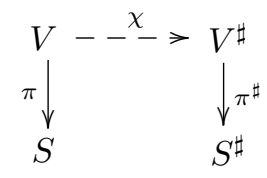

где $\pi^{\sharp}: V^{\sharp} \rightarrow S^{\sharp}-$ расслоение на многообразия отрицательной кодаировой размерности, является послойным, т. е. существует рациональное доминантное отображение $\rho: S \rightarrow S^{\sharp}$, превращающее диаграмму (6) в коммутативную, $\pi^{\sharp} \circ \chi=\rho \circ \pi$. Другими словами, $\pi^{\sharp} \geqslant \pi$ в смысле отношения порядка на множестве рационально связных структур: $\pi$ - минимальный элемент $R C(V)$.

Таким образом, для некоторых рационально связных многообразий виртуальный порог канонического присоединения сводит проблему описания множества $R C(V)$ к такой же проблеме для базы $S$. Это - ключевой шаг, во многих случаях ведущий к исчерпывающему описанию множества $R C(V)$. Но главный недостаток виртуальных порогов состоит в том, что их очень трудно вычислять.

Точнее говоря, единственный известный способ их вычислять - сводить к обычным порогам. 
ОПРЕДЕЛЕНИЕ 2.3. (i) Многообразие $V$ называется бирационалъно сверхжестким, если для любой подвижной линейной системы $\Sigma$ на $V$ выполнено равенство

$$
c_{\text {virt }}(\Sigma)=c(\Sigma, V) .
$$

(ii) Многообразие $V$ (соответственно расслоение Фано $V / S$ ) называется бирационально жестким, если для любой подвижной линейной системы $\Sigma$ на $V$ существует бирациональный автоморфизм $\chi \in \operatorname{Bir} V$ (соответственно послойный бирациональный автоморфизм $\chi \in \operatorname{Bir}(V / S)$ ), обеспечивающий равенство

$$
c_{\mathrm{virt}}(\Sigma)=c\left(\chi_{*} \Sigma, V\right) .
$$

В следующих примерах перечислены основные классы многообразий и расслоений Фано, для которых сегодня известна бирациональная жесткость или сверхжесткость.

ПримеР 2.3. (i) Гладкая трехмерная квартика $V=V_{4} \subset \mathbb{P}^{4}$ бирационально сверхжесткая, что немедленно следует из рассуждений в [3].

(ii) Общее гладкое полное пересечение $V_{2 \cdot 3} \subset \mathbb{P}^{5}$ кубической гиперповерхности и квадрики бирационально жесткое, но не сверхжесткое [16], [78]. Описание его группы бирациональных автоморфизмов обсуждается ниже, в пп. 3.2, 3.3 настоящей главы.

(iii) Общее полное пересечение $V_{d_{1} \cdots d_{k}} \subset \mathbb{P}^{M+k}$ индекса 1 (т. е. $d_{1}+\cdots+d_{k}=$ $M+k)$ и размерности $M \geqslant 4$ бирационально сверхжесткое при $M \geqslant 2 k+1$ [67].

(iv) Первыми примерами бирационально сверхжестких многообразий Фано произвольной размерности были двойные пространства $\sigma: V \stackrel{2: 1}{\longrightarrow} \mathbb{P}^{M}, M \geqslant 4$, разветвленные над гладкой гиперповерхностью $W_{2 M} \subset \mathbb{P}^{M}$ степени $2 M$, и двойные квадрики $\sigma: V \stackrel{2: 1}{\longrightarrow} Q \subset \mathbb{P}^{M+1}, M \geqslant 4$, разветвленные над гладким полным пересечением $W=Q \cap W_{2(M-1)}^{*}, W_{2(M-1)}^{*} \subset \mathbb{P}^{M+1}$ - гиперповерхность степени $2(M-1)$ [60].

(v) Обобщая предыдущий пример, пусть $\sigma: V \rightarrow Q \subset \mathbb{P}^{M+1}-$ двойное накрытие, где $Q=Q_{m} \subset \mathbb{P}^{M+1}$ - гладкая гиперповерхность степени $m$, а дивизор ветвления $W \subset Q$ высекается на $Q$ гиперповерхностью $W_{2 l}^{*} \subset \mathbb{P}^{M+1}$, где $m+l=M+1$. Многообразие $V$ бирационально сверхжесткое для общих $Q$, $W^{*}[65]$. Вместо двойного накрытия можно рассмотреть произвольное циклическое накрытие, вместо гиперповерхности $Q \subset \mathbb{P}^{M+1}$ - гладкое полное пересечение $Q \subset \mathbb{P}^{M+k}$ подходящего индекса и коразмерности $k<(1 / 2) M$. Общее многообразие в этих классах бирационально сверхжесткое [73], [76]. Другой пример дают итерированные двойные накрытия [70]. Все многообразия примера 2.3 реализуются как полные пересечения Фано во взвешенных проективных пространствах.

ГиПотезА 2.1. Гладкое полное пересечение Фано индекса единица и размерности $\geqslant 4$ во взвешенном проективном пространстве бирационально жесткое, размерности $\geqslant 5$ - бирачионально сверхжесткое.

В заключение еще раз напомним, что для примитивного многообразия Фано бирациональная (сверх)жесткость немедленно влечет отсутствие нетривиальных структур рационально связного расслоения. 
1.2. Особенности линейных систем. Исходная точка доказательства бирациональной (сверх)жесткости рационально связного многообразия $V$ такова: зафиксируем подвижную линейную систему $\Sigma$, удовлетворяющую неравенству

$$
c_{\text {virt }}(\Sigma)<c(\Sigma)
$$

(если таких систем нет, то многообразие сверхжесткое и доказывать нечего). По определению это означает, что существует бирациональный морфизм $\varphi: V^{+} \rightarrow V$, для которого выполнено неравенство $c\left(\Sigma^{+}, V^{+}\right)<c(\Sigma)$, где $\Sigma^{+}-$ собственный прообраз системы $\Sigma$. Отметим, что $\varphi$ не может быть изоморфизмом в коразмерности 1 (т. е. вне замкнутого подмножества $Y_{+} \subset V^{+}$коразмерности $\geqslant 2)$ : в противном случае для любого эффективного дивизора $D$ на $V$ и его собственного прообраза $D^{+}$было бы выполнено равенство $c(D)=c\left(D^{+}\right)$. Стало быть, существуют исключительные дивизоры $E \subset V^{+}$, стягиваемые морфизмом $\varphi$ (в классической терминологии исключительным дивизором называется объединение всех неприводимых, или простых, дивизоров, стягиваемых морфизмом $\varphi$; для удобства изложения мы называем каждую неприводимую компоненту также исключительным дивизором). Каждый такой дивизор определяет дискретное нормирование $\operatorname{ord}_{E}(\cdot)$ поля рациональных функций $\mathbb{C}(V)$. Это нормирование не зависит от модели $V^{+}$в следующем смысле: если $\varphi^{\sharp}: V^{\sharp} \rightarrow V-$ другой бирациональный морфизм, причем бирациональное отображение $\left(\varphi^{\sharp}\right)^{-1} \circ \varphi: V^{+} \rightarrow V^{\sharp}$ есть изоморфизм в общей точке дивизора $E$, так что $\left(\varphi^{\sharp}\right)^{-1} \circ \varphi(E)=E^{\sharp} \subset V^{\sharp}-$ исключительный дивизор морфизма $\varphi^{\sharp}$, то $\operatorname{ord}_{E}=\operatorname{ord}_{E \sharp}$. Неприводимое многообразие $\varphi(E) \subset V$ называется центром дискретного нормирования $\operatorname{ord}_{E}$ и также не зависит от выбора модели $V^{+}$(обозначение: $\operatorname{centre}(E, V)$ или просто centre $(E)$, когда ясно, о какой модели идет речь). Нормирования поля $\mathbb{C}(V)$, реализуемые исключительными дивизорами бирациональных морфизмов, называются геометрическими.

Поскольку дивизор $D$ на многообразии $V$ задается локальными уравнениями, то применяя нормирование $\operatorname{ord}_{E}$, получаем кратность $\nu_{E}(D) \in \mathbb{Z}_{+}$эффективного дивизора $D$ относительно $E$. Очевидно, что $\nu_{E}(D) \geqslant 1$ тогда и только тогда, когда носитель дивизора $D$ содержит centre $(E)$. Если $\mathscr{E}$ - множество исключительных дивизоров бирационального морфизма $\varphi, D^{+}-$собственный прообраз $D$ на $V^{+}$, то имеем

$$
\varphi^{*} D=D^{+}+\sum_{E \in \mathscr{E}} \nu_{E}(D) E .
$$

Для канонического класса $K_{V+}$ имеем представление

$$
K_{V^{+}}=\varphi^{*} K_{V}+\sum_{E \in \mathscr{E}} a(E) E,
$$

где $a(E)=a(E, V) \geqslant 1$ - дискрепантность геометрического нормирования $E$, также не зависящая от модели $V^{+}$. Напомним, что мы работаем в классе неособых проективных многообразий.

В силу предположения имеем $n=c(\Sigma)>0$. 
ОПРЕДЕЛЕНИЕ 2.4. Геометрическое дискретное нормирование $\operatorname{ord}_{E}$ поля рациональных функций $\mathbb{C}(V)$ называется максимальной особенностью линейной системы $\Sigma$, если выполнено неравенство Нётера-Фано

$$
\nu_{E}(\Sigma)>n a(E)
$$

где $\nu_{E}(\Sigma)=\nu_{E}(D)$ для общего дивизора $D \in \Sigma$.

Имеется особенно простой и важный класс максимальных особенностей.

ОПРЕДЕЛЕНИЕ 2.5. Неприводимое подмногообразие $Y \subset V$ коразмерности $\geqslant 2$ называется максимальным подмногообразием линейной системы $\Sigma$, если выполнено неравенство

$$
\operatorname{mult}_{Y} \Sigma>n(\operatorname{codim} Y-1)
$$

где mult $_{Y} \Sigma=$ mult $_{Y} D$ для общего дивизора $D \in \Sigma$.

Отметим, что для корректного определения чисел $\nu_{E}(D)$ и $a(E)$ достаточно, чтобы многообразие $V^{+}$было неособым лишь в общей точке исключительного дивизора $E$. Например, в определениях и конструкциях, данных выше, можно предполагать лишь, что особенности многообразия $V^{+}$имеют коразмерность $\geqslant 2$ (это так, если $V^{+}$нормально). Имея это в виду, предположим, что $Y \subset V$ - максимальное подмногообразие линейной системы $\Sigma$. Раздуем его: $\varphi: V^{+} \rightarrow V$, пусть $E=\varphi^{-1}(Y)$ - исключительный дивизор. Имеем $\nu_{E}(\Sigma)=\operatorname{mult}_{Y} \Sigma$ и $a(E)=\operatorname{codim} Y-1$, так что $E$ реализует максимальную особенность системы $\Sigma$. Поэтому максимальные подмногообразия представляют собой (простейший) тип максимальных особенностей.

ПРЕДЛОЖЕНИЕ 2.2. В предположении, что выполнено неравенство (7), линейная система $\Sigma$ обладает максимальной особенностью.

ДокАзАтельство. Пусть $\varphi: V^{+} \rightarrow V$ - бирациональный морфизм (с неособым $\left.V^{+}\right)$, удовлетворяющий неравенству $c\left(\Sigma^{+}\right)<c(\Sigma)=n, \mathscr{E}-$ множество простых дивизоров, стягиваемых морфизмом $\varphi, D \in \Sigma-$ общий дивизор, $D^{+} \in \Sigma^{+}-$его собственный прообраз на $V^{+}$. Из соотношений (8), (9) получаем

$$
A_{+}^{1} V^{+} \not \supset D^{+}+n K_{V^{+}}=\varphi^{*}\left(D+n K_{V}\right)-\sum_{E \in \mathscr{E}} e(E) E,
$$

где $e(E)=\nu_{E}(D)-n a(E)$. Поскольку $D+n K_{V} \in A_{+}^{1} V$ и подъем псевдоэффективного класса псевдоэффективен, получаем, что существует хотя бы один исключительный дивизор $E$, для которого $e(E)>0$, что и требовалось доказать.

Отметим, что в силу сделанного выше замечания многообразие $V^{+}$достаточно предполагать неособым в коразмерности 1, так что предложение 2.2 не зависит от теоремы о разрешении особенностей и поэтому верно в любой характеристике. 
С исключительным дивизором $E \subset V^{+}$связана однозначно определенная последовательность раздутий. Пусть $X$ - некоторое проективное (возможно, особое) многообразие, $\psi: V^{+} \rightarrow X-$ бирациональное отображение, стягивающее $E$ в подмногообразие $B=\psi(E) \subset X$ коразмерности $\geqslant 2$, причем $B \not \subset \operatorname{sing} X$ не содержится целиком во множестве особых точек. Пусть $\sigma_{B}: X(B) \rightarrow X-$ раздутие подмногообразия $B, E(B)=\sigma_{B}^{-1}(B)-$ исключительный дивизор.

ПРЕДЛОЖЕНИЕ 2.3. Имеет место альтернатива: либо композииия бираииональных отображений $\sigma_{B}^{-1} \circ \psi: V^{+} \rightarrow X$ есть изоморбизм в окрестности общей точки дивизора $E$ и тогда $\sigma_{B}^{-1} \circ \psi(E)=E(B)$, либо $B^{+}=\sigma_{B}^{-1} \circ \psi(E)$ есть неприводимое подмногообразие коразмерности $\geqslant 2$, причем $B^{+} \not \subset \operatorname{Sing} X(B)$, $B^{+} \subset E(B) u \sigma_{B}\left(B^{+}\right)=B$.

ДокАЗАТЕЛЬСтво. Это очевидно: мы просто перечислили имеющиеся возможности. Отметим лишь, что многообразие $X(B)$ неособо вне $\sigma_{B}$-прообраза множества $\operatorname{Sing} X \cup \operatorname{Sing} B$ и $\sigma_{B}\left(B^{+}\right)=\sigma_{B} \circ \sigma_{B}^{-1} \circ \psi(E)=B$. Отметим также, что вне $\sigma_{B}$-прообраза множества $\operatorname{Sing} X \cup \operatorname{Sing} B$ морфизм $\sigma_{B}: E(B) \rightarrow B$ есть локально-тривиальное $\mathbb{P}^{\operatorname{codim} B-1}$-расслоение, а дискрепантность исключительного дивизора $E(B)$ есть $\operatorname{codim} B-1$.

Итерируя конструкцию предложения 2.3 , получаем последовательность раздутий

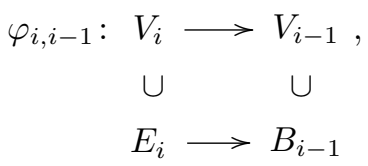

$i=1,2, \ldots$, где $V_{0}=V, B_{0}=\operatorname{centre}(E, V)$ и, далее, $B_{j}$ есть центр $E$ на $V_{j}$, $E_{i}=\varphi_{i, i-1}^{-1}\left(B_{i-1}\right)-$ исключительный дивизор, $B_{i-1}-$ центр раздутия $\varphi_{i, i-1}$. Иными словами, мы последовательно раздуваем центры нормирования $E$. Многообразия $V_{1}, V_{2}, \ldots$, могут, вообще говоря, быть особыми, однако $V_{j}$ неособо в общей точке подмногообразий $B_{j} \subset E_{j}$ для каждого $j=1,2, \ldots$. Положим для $i>j$

$$
\varphi_{i, j}=\varphi_{j+1, j} \circ \cdots \circ \varphi_{i, i-1}: V_{i} \rightarrow V_{j}
$$

$\varphi_{i, i}=\operatorname{id}_{V_{i}}$. Согласно (итерированному) предположению $2.3, \varphi_{i, j}\left(B_{i}\right)=B_{j}$ при $i>j$. Для неприводимого подмногообразия на $V_{j}$ (скажем, $Y \subset V_{j}$ ) его собственный прообраз на $V_{i}$ (если он определен, т. е. $Y \not \subset B_{j}$ ) обозначаем добавлением верхнего индекса $i$ (скажем, $Y^{i} \subset V_{i}$ ).

То же обозначение применяем к эффективным алгебраическим циклам, скажем, $Z^{i}=\sum m_{k} Z_{k}^{i}$ для цикла $Z=\sum m_{k} Z_{k}$ на $V_{j}$. Использование этого обозначения подразумевает, что собственный прообраз корректно определен.

ПРЕДЛОЖЕНИЕ 2.4. Последователъность раздутий (11) обрывается: для некоторого $K \geqslant 1$ реализуется первый случай альтернативы предложения 2.3, m.e. $\sigma_{K, 0}^{-1} \circ \psi(E)=E_{K}$. 
ДокАЗАТЕЛЬСтво. Как мы увидим ниже, дискрепантности исключительных дивизоров $E_{i}$ по отношению к модели $V$ монотонно возрастают, в частности, $a\left(E_{i}, V\right) \geqslant i$, в то же время $a\left(E_{i}, V\right) \leqslant a(E, V)$, поскольку центр $E$ на $V_{i}$ содержится в $E_{i}$, что и требовалось доказать.

Последовательность раздутий (11) назовем разрешением нормирования $\nu_{E}$ относительно модели $V$. На множестве раздутий $\varphi_{i, i-1}$ или, точнее, множестве исключительных дивизоров $\left\{E_{1}, \ldots, E_{K}\right\}$ введем структуру ориентированного графа следующим образом: положим $i \rightarrow j$, если $i>j$ и $B_{i-1} \subset E_{j}^{i-1}$. Эта важная структура впервые появилась в [3] (для поверхностей - в [13]). Ее смысл в том, чтобы вычислять собственный прообраз исключительных дивизоров:

$$
E_{j}^{i}=\varphi_{i, j}^{*} E_{j}-\sum_{j \leftarrow k \leqslant i} \varphi_{i, k}^{*} E_{k} .
$$

Для того, чтобы, наоборот, вычислить полный прообраз в терминах собственных прообразов, положим для $i>j: p_{i j}$ - число путей из $E_{i}$ в $E_{j}$ в описанном ориентированном графе, $p_{i j} \geqslant 1$. Положим также $p_{i i}=1$.

ПРЕДЛОЖЕНИЕ 2.5. Имеет место разложение

$$
\varphi_{i, j}^{*} E_{j}=\sum_{k=j}^{i} p_{k j} E_{k}^{i}
$$

ДоКАЗАТЕЛЬСтво (следуя [3]) проводится индукцией по $i \geqslant j$. Если $i=j$, то доказывать нечего. Если $i=j+1$, то $\varphi_{j+1, j}^{*} E_{j}=E_{j}^{j+1}+E_{j+1}$, поскольку $B_{j} \subset E_{j}$ и дивизор $E_{j}$ неособ в общей точке $B_{j}$. Теперь для $i \geqslant j+2$ имеем:

$$
\begin{gathered}
\varphi_{i, j}^{*} E_{j}=\varphi_{i, i-1}^{*}\left(\varphi_{i-1, j}^{*} E_{j}\right)=\varphi_{i, i-1}^{*}\left(\sum_{k=j}^{i-1} p_{k j} E_{k}^{i-1}\right) \\
=\sum_{k=j}^{i-1} p_{k j} E_{k}^{i}+\left(\sum_{\substack{k=j \\
B_{i-1} \subset E_{k}^{i-1}}}^{i-1} p_{k j}\right) E_{i} .
\end{gathered}
$$

Нетрудно понять, что имеет место равенство

$$
p_{i j}=\sum_{i \rightarrow k} p_{k j}
$$

(в каждом пути из $i$ в $j$ отметим первую вершину после $i: i \rightarrow k \rightarrow \cdots \rightarrow j$ ). Этим предложение доказано.

Комбинаторные варианты $p_{i j}$ дают явные представления для кратностей и дискрепантностей. Пусть $\Sigma^{j}-$ собственный прообраз линейной системы $\Sigma$ на $V_{j}$. Положим $\nu_{j}=$ mult $_{B_{j-1}} \Sigma^{j-1}$ и $\delta_{j}=\operatorname{codim} B_{j-1}-1$. Получаем

$$
\nu_{E_{K}}(\Sigma)=\nu_{E}(\Sigma)=\sum_{i=1}^{K} p_{K i} \nu_{i}, \quad a(E)=\sum_{i=1}^{K} p_{K i} \delta_{i} .
$$


Полагая для удобства обозначений $p_{i}=p_{K i}$, получаем традиционную форму неравенства Нётера-Фано:

$$
\sum_{i=1}^{K} p_{i} \nu_{i}>n \sum_{i=1}^{K} p_{i} \delta_{i} .
$$

ЗАмЕчАниЕ 2.1. Из проведенных рассуждений видно, что наши построения имеют смысл в более общем контексте. Многообразие $V$ может быть особым, однако необходимо, во-первых, чтобы для любого дивизора Вейля $D$ на $V$ по крайней мере некоторая его кратность $N D$ задавалась локально одним уравнением (это позволяет поднимать дивизоры относительно морфизмов) и, во-вторых, чтобы для любых раздутий все дискрепантности исключительных дивизоров были положительны. Таким образом, неравенство Нётера-Фано и понятие максимальной особенности имеют смысл для многообразий с $\mathbb{Q}$-факториальными терминальными особенностями.

ЗАмечАниЕ 2.2. Неравенство Нётера-Фано может быть следующим образом переформулировано на языке $\mathbb{Q}$-дивизоров (т. е. линейных комбинаций простых дивизоров с рациональными коэффициентами). Пусть $D \in \Sigma$ - общий дивизор. Тогда логпара $(V,(1 / n) D)$ не канонична, т. е. имеет неканоническую особенность $E \subset V^{+}$, удовлетворяющую неравенству $\nu_{E}((1 / n) D)>a(E)$. Напомним [30], что логпара $(V, Z)$, где $Z$ - эффективный $\mathbb{Q}$-дивизор, канонична, если для любого геометрического дискретного нормирования $\nu_{E}$ имеет место неравенство $\nu_{E}(Z) \leqslant a(E)$. (Таким образом, канонические особенности пар, столь популярные в наши дни, фактически были открыты в [3] в форме максимальных особенностей линейных систем.) Пара $(V, Z)$ терминальна (соответственно логтерминальна и логканонична), если для любого $\nu_{E}$ имеет место неравенство $\nu_{E}(Z)<a(E)$ (соответственно $\nu_{E}(Z)<a(E)+1$ и $\left.\nu_{E}(Z) \leqslant a(E)+1\right)$. Эти понятия работают в современных продвинутых методах многомерной бирациональной геометрии.

В качестве иллюстрации этих концепций докажем следующее предложение.

ПреДЛОЖениЕ 2.6. Пусть $V$ - примитивное многообразие Фано, $V^{\prime}$ - многообразие Фано с $\mathbb{Q}$-факториальными терминальными особенностями и числом Пикара единица, т.е. $\operatorname{Pic} V^{\prime} \otimes \mathbb{Q}=\mathbb{Q} K_{V^{\prime}}, \chi: V \rightarrow V^{\prime}-$ бирациональное отображение.

(i) Предположим, что $V$ бирационально жесткое. Тогда $V$ u $V^{\prime}$ (бирегулярно) изоморфны (хотя само отображение $\chi$, вообще говоря, не есть изоморфизм).

(ii) Предположим, что $V$ бирачионально сверхжесткое. Тогда ұ есть бирегулярный изоморфизм. В частности, группь бирациональных и бирегулярных автоморфизмов многообразия $V$ совпадают: $\operatorname{Bir} V=\operatorname{Aut} V$.

ДокАЗАТЕльство. (i) Пусть $\chi: V \rightarrow V^{\prime}-$ бирациональное отображение, $\varphi: Y \rightarrow V$ - его разрешение по Хиронаке, так что $\psi=\chi \circ \varphi: Y \rightarrow V^{\prime}$ - бирациональный морфизм. Многообразие $Y$ неособо и

$$
\operatorname{Pic} Y=\mathbb{Z} \varphi^{*} K_{V} \oplus \bigoplus_{i \in I} \mathbb{Z} E_{i},
$$


где $\left\{E_{i} \mid i \in I\right\}$ - множество всех $\varphi$-исключительных дивизоров. По предположению,

$$
\operatorname{Pic} Y \otimes \mathbb{Q}=\mathbb{Q} \psi^{*} K_{V^{\prime}} \oplus \bigoplus_{j \in J} \mathbb{Q} E_{j}^{\prime},
$$

где $\left\{E_{j}^{\prime} \mid j \in J\right\}$ - множество всех $\psi$-исключительных дивизоров. Для простоты обозначений положим $K=\varphi^{*} K_{V}, K^{\prime}=\psi^{*} K_{V^{\prime}}$. Получаем

$$
K_{Y}=K+\sum_{i \in I} a_{i} E_{i}=K^{\prime}+\sum_{j \in J} a_{j}^{\prime} E_{j}^{\prime},
$$

где $a_{i} \in \mathbb{Z}, a_{i} \geqslant 1$ и $a_{j}^{\prime} \in \mathbb{Q}, a_{j}^{\prime}>0$. Пусть $\Sigma^{\prime}=\left|-m K_{V^{\prime}}\right|, m \gg 0,-$ очень обильная линейная система. Очевидно, что $c\left(\Sigma^{\prime}, V^{\prime}\right)=m$. Возьмем ее собственный прообраз, $\Sigma=\chi_{*}^{-1} \Sigma^{\prime} \subset\left|-n K_{V}\right|$; очевидно, что $c(\Sigma, V)=n$. Подкручивая на подходящий бирациональный автоморфизм, мы можем считать, что неравенство (7) выполнено уже для $\chi$. Значит, $n \leqslant m$. Собственный прообраз линейной системы $\Sigma$ на $Y$ совпадает с собственным прообразом линейной системы $\Sigma^{\prime}$ относительно $\psi$. Следовательно, существуют целые положительные числа $b_{i}, i \in I$, такие, что

$$
-m K^{\prime}=-n K-\sum_{i \in I} b_{i} E_{i}
$$

Деля на $m$ и подставляя в (14), получаем:

$$
\left(1-\frac{n}{m}\right) K=\sum_{i \in I}\left(\frac{b_{i}}{m}-a_{i}\right) E_{i}+\sum_{j \in J} a_{j}^{\prime} E_{j}^{\prime} .
$$

Поскольку дивизоры $E_{i}$ являются $\varphi$-исключительными и $a_{j}^{\prime}>0$, получаем равенство $n=m$ : иначе - противоречие с обильностью класса $\left(-K_{V}\right)$. Далее, все дивизоры $E_{j}^{\prime}$ оказываются $\varphi$-исключительными и, более того, $\left\{E_{i} \mid i \in I\right\}=$ $\left\{E_{j}^{\prime} \mid j \in J\right\}$, иначе $\operatorname{rk} \operatorname{Pic} V^{\prime} \geqslant 2$. Значит, $\chi-$ изоморфизм в коразмерности единица: положим

$$
U=V \backslash \bigcup_{i \in I} \varphi\left(E_{i}\right), \quad U^{\prime}=V^{\prime} \backslash \bigcup_{j \in J} \psi\left(E_{j}^{\prime}\right),
$$

тогда $\chi: U \rightarrow U^{\prime}-$ изоморфизм. Следовательно, $\Sigma=\left|-n K_{V}\right|$ и $\chi$ индуцирует изоморфизм линейных систем $\Sigma$ и $\Sigma^{\prime}$, так что $\chi: V \rightarrow V^{\prime}-$ изоморфизм. (Строго говоря, доказано, что для произвольного бирационального отображения $\chi: V \rightarrow V^{\prime}$ существует такой бирациональный автоморфизм $\chi^{*} \in \operatorname{Bir} V$, что $\chi \circ \chi^{*}-$ изоморфизм.)

Утверждение (ii) теперь очевидно. Предложение 2.6 доказано.

1.3. Общая схема метода. Имея определение максимальной особенности, можем точно сформулировать основные шаги метода максимальных особенностей, описанного в п. 3.3 главы 1. Для каждого геометрического дискретного нормирования $\nu=\nu_{E}$ поля $\mathbb{C}(V)$ (реализуемого исключительным дивизором $E \subset V^{+}$на некоторой модели $V^{+} \rightarrow V$ ) нужно ответить на вопрос: существует ли подвижная линейная система $\Sigma$ с порогом канонического присоединения 
$n=c(\Sigma)>0$, для которой $\nu_{E}$ есть максимальная особенность (m.е. имеет место неравенство (10))?

Получение отрицательного ответа на этот вопрос называется исключением данной максимальной особенности. Как мы отметили выше, это наиболее трудная часть работы. Известные сегодня технические приемы исключения рассмотрены подробно ниже.

Если ответ на поставленный выше вопрос положительный, то необходимо предъявить подвижную линейную систему $\Sigma$ с максимальной особенностью $E$. Следующий шаг в этом случае - откручивание максимальной особенности $E$. В наиболее простом виде процедура откручивания выглядит так: строится бирациональный автоморфизм $\tau_{E} \in \operatorname{Bir} V$ такой, что для любой линейной системы $\Sigma$, имеющей максимальную особенность $E$, выполнено неравенство

$$
c\left(\left(\tau_{E}^{-1}\right)_{*} \Sigma\right)<c(\Sigma)
$$

где $\left(\tau_{E}^{-1}\right)_{*} \Sigma$ - собственный прообраз $\Sigma$ относительно $\tau_{E}$, причем $E$ уже не является максимальной особенностью системы $\left(\tau_{E}^{-1}\right)_{*} \Sigma$. Таким образом, $\tau_{E}$ "откручивает" (устраняет) максимальную особенность $E$, одновременно понижая порог канонического присоединения.

Пусть $V$ - примитивное многообразие Фано. Предположим, что для $V$ описанная выше схема успешно реализована. Пусть $\mathscr{M}$ - множество дискретных нормирований, для которых ответ на поставленный выше вопрос положительный, т. е. любое нормирование $E$ реализуется в качестве максимальной особенности тогда и только тогда, когда $E \in \mathscr{M}$.

В этих предположениях имеет место следующая теорема.

Теорема 2.1. (i) Многообразие $V$ бирационально жесткое.

(ii) Группа бирационалъных автоморфизмов $\operatorname{Bir} V$ порождена подгруппой бирегулярных автоморфизмов Aut $V$ и подгруппой $B(V)$, порожденной откручивающими преобразованиями $\tau_{E}, E \in \mathscr{M}$.

(iii) Если $\mathscr{M}=\varnothing$, то $V$ бирационально сверхжесткое.

ДокАЗАТЕЛЬСтво. На примитивном многообразии Фано порог канонического присоединения принимает лишь целые положительные значения. Поэтому процедура откручивания не может быть бесконечной: для некоторой последовательности максимальных особенностей $E_{1}, \ldots, E_{N}$ бирациональный автоморфизм $\tau=\tau_{E_{1}} \circ \cdots \circ \tau_{E_{N}}$ преобразует систему $\Sigma$ в систему $\Sigma=\left(\tau^{-1}\right)_{*} \Sigma$, не имеющую максимальных особенностей, и потому, согласно предложению 2.2 ,

$$
c\left(\Sigma^{\tau}\right)=c_{\mathrm{virt}}\left(\Sigma^{\tau}\right)=c_{\mathrm{virt}}(\Sigma),
$$

что и означает бирациональную жесткость. Если $\mathscr{M}=\varnothing$, то получаем это равенство порогов сразу, что и доказывает (iii). Осталось установить (ii). Для произвольного бирационального автоморфизма $\chi \in \operatorname{Bir} V$ рассмотрим очень обильную линейную систему $\Sigma^{*}=\left|-m K_{V}\right|$ и ее собственный прообраз $\Sigma^{\chi}=$ $\left(\chi^{-1}\right)_{*} \Sigma^{*}$ относительно $\chi$. Если $c\left(\Sigma^{\chi}\right)=c_{\text {virt }}\left(\Sigma^{\chi}\right)=c_{\text {virt }}\left(\Sigma^{*}\right) \leqslant m$, то $\Sigma^{\chi}=\Sigma^{*}$ (скажем, по соображениям размерности $\operatorname{dim} \Sigma^{\chi}$ ), так что $\chi \in \operatorname{Aut} V$ - бирегулярный автоморфизм. Теорема доказана. (Мы видим, что процедура откручивания максимальных особенностей доказывает (ii) одновременно с (i).) 
ЗАмЕчАниЕ 2.3. Во всех изученных случаях откручивающие бирациональные отображения $\tau_{E}$ являются инволюциями, канонически определенными соответствующими максимальными особенностями, так что, в частности, для любого бирегулярного автоморфизма $\alpha \in \operatorname{Aut} V$ имеет место равенство $\alpha^{-1} \tau_{E} \alpha=$ $\tau_{\alpha(E)}$ и подгруппа $B(V)$ нормальна, a $\operatorname{Bir} V$ есть расширение

$$
1 \rightarrow B(V) \rightarrow \operatorname{Bir} V \rightarrow \text { Aut } V \rightarrow 1
$$

Далее, из описания процедуры откручивания понятно, что соотношения между образующими $\tau_{E}, E \in \mathscr{M}$, могут появляться тогда и только тогда, когда откручивание не определено однозначно, т. е. некоторая подвижная линейная система $\Sigma$ может иметь две различные максимальные особенности $E_{1} \neq E_{2}$ одновременно. Если, наоборот, такое невозможно, то группа $B(V)$ есть свободное произведение циклических групп $\left\langle\tau_{E}\right\rangle$ (в частности, очень велика). Приведенное рассуждение дает несколько упрощенную картину. Точное описание дано ниже в $\S 3$ на примере вычисления группы бирациональных автоморфизмов $\operatorname{Bir} V_{2 \cdot 3}$ полного пересечения Фано $V_{2 \cdot 3} \subset \mathbb{P}^{5}$.

ЗАмЕчАниЕ 2.4. Описанная выше процедура откручивания максимальных особенностей является удовлетворительной далеко не для всех многообразий. Типичной является ситуация, когда некоторая максимальная особенность $E$ реализуется подвижной линейной системой $\Sigma$, однако не откручивается бирациональным автоморфизмом: такого автоморфизма $\tau_{E}$ просто не существует. Опыт практического изучения таких особенностей уже давно привел к общему убеждению, что в этом случае особенности $E$ соответствует другая струкmура рационально связного расслоения или многообразия Фано на $V$, т. е. $E$ откручивается бирациональным отображением $V \rightarrow V^{\sharp}$. Эти соображения, основанные на большом эмпирическом материале, составляют суть программы Саркисова - теории факторизации бирациональных отображений в композицию бирациональных отображений очень специального вида - элементарных линков [79]. В размерности три программа Саркисова есть полностью доказанная теорема, много раз публиковавшаяся в разной степени подробности [1], [2], [17], [80]. Недавние успехи программы логминимальных моделей [28], [29] позволяют надеяться, что и многомерная версия программы Саркисова будет завершена в обозримом будущем. Однако подчеркнем, что это - общая теорема существования, сама по себе не дающая описания бирациональной геометрии конкретного многообразия $V$. Как и сто лет тому назад, главная проблема заключается в выделении реализуемых максимальных особенностей и в построении откручивающих отображений (последнее для явно заданного многообразия никогда не является сложной задачей). По этой причине в данном обзоре мы ограничились простым вариантом метода максимальных особенностей, когда откручивание осуществляется бирациональными автоморфизмами. Кроме того, следует иметь в виду, что примеров до конца изученных многообразий (за исключением расслоений на коники), в которых появляются линки на другую модель, очень мало [51], [57], [74], [81]: как правило, многообразия с нетривиальными линками имеют богатую бирациональную геометрию и современная техника еще недостаточно сильна для них. 
ЗАмЕчАниЕ 2.5. Выше мы упомянули некоторые варианты понятия максимальной особенности, пришедшие из программы минимальных моделей. Если нормирование $\nu_{E}$ удовлетворяет нестрогому неравенству Нётера-Фано, т. е. $\nu_{E}(\Sigma) \geqslant n a(E)$, то пара $(V,(1 / n) D)$ не терминальна. Знание таких особенностей $E$ (фактически речь идет о равенстве Нётера-Фано, $\nu_{E}=n a(E)$, так как иначе получаем обычное понятие максимальной особенности) позволяет описывать структуры $K$-тривиального расслоения на $V$, т. е. бирациональные отображения $\chi: V \rightarrow V^{\sharp}$, где $V^{\sharp}$ снабжено морфизмом $\pi: V^{\sharp} \rightarrow S^{\sharp}$, слой общего положения которого имеет тривиальный канонический класс, а также бирациональные отображения $\chi: V \rightarrow V^{\sharp}$ на многообразия Фано с каноническими особенностями [2], [52]-[56], [67], [75]. В частности, несложная модификация доказательства предложения 2.6 (в конце рассуждения нужно учесть, что исключительные дивизоры $E_{i}$ появятся со строго положительными коэффициентами) дает следующее утверждение.

ПРЕДЛОЖЕНИЕ 2.7. Предположим, что на примитивном многообразии Фано $V$ нет максимальных особенностей, $\mathscr{M}=\varnothing$, а любая подвижная линейная система $\Sigma$, удовлетворяющая нестрогому неравенству Нётера-Фано для некоторого $\nu_{E}$, задает отображение $V \rightarrow S$ на многообразие меньшей размерности. Тогда любое бирациональное отображение $\chi: V \rightarrow V^{\sharp}$ на многообразие $V^{\sharp}$ с каноническими особенностями (без ограничений на ранг группь Пикара $\left.\rho\left(V^{\sharp}\right)\right)$ есть бирегулярньй изоморфизм.

\section{§ 2. Исключение максимальных особенностей}

В этом параграфе рассмотрена задача исключения максимальной особенности $E \subset V^{+}$подвижной линейной системы $\Sigma$ на гладком многообразии $V$. Задача естественно распадается на исключение максимальных подмногообразий (п. 2.1) и исключение бесконечно близкой максимальной особенности. В п. 2.2 рассмотрен ключевой локальный факт, на котором основана процедура исключения - $4 n^{2}$-неравенство, в п. 2.3 приведено другое доказательство этого неравенства, основанное на принципе связности Шокурова-Коллара.

2.1. Максимальные подмногообразия. Мы используем обозначения и конструкции п. 1.2. Особенность $E \subset V^{+}$и ее разрешение фиксированы. Мы хотим доказать, что $E$ не является максимальной особенностью никакой подвижной линейной системы $\Sigma$ с $n=c(\Sigma)>0$. Методы доказательства существенно различны для разных типов особенностей и разных классов многообразий. Прежде всего, разделим множество особенностей на два естественных типа. Размерности раздуваемых подмногообразий $\operatorname{dim} B_{i}, i=0,1, \ldots, N-1$, не убывают. Следовательно, имеет место альтернатива: либо $\operatorname{dim} B_{0}=\cdots=$ $\operatorname{dim} B_{N-1}$, либо $\operatorname{dim} B_{0}<\operatorname{dim} B_{N-1}$. В последнем случае скажем, что $E-$ бесконечно близкая особенность.

ПреДЛОжЕНИЕ 2.8. Предположим, что $E$ - максимальная особенность подвижной линейной системы $\Sigma u \operatorname{dim} B_{0}=\operatorname{dim} B_{N-1}$. Тогда подмногообразие $B=B_{0}$ (центр особенности $E$ на $V$ ) есть максимальное подмногообразие cистемы $\Sigma$. 
ДокАЗАТЕльство. Утверждение очевидным образом следует из явной формы (13) неравенства Нётера-Фано, равенства локальных дискрепантностей $\beta_{1}=\cdots=\beta_{N}=\operatorname{codim} B-1$ и невозрастания кратностей $\nu_{1} \geqslant \nu_{2} \geqslant \cdots \geqslant \nu_{N}$.

СЛЕДСТВИЕ 2.1. Если центр $B \subset V$ максималъной особенности $E$ не есть максимальное подмногообразие системы $\Sigma$, то $E$ - бесконечно близкая максимальная особенность.

Наибольшую трудность представляет именно исключение бесконечно близкого случая. Сейчас рассмотрим основные приемы исключения максимальных подмногообразий. Чаще всего используются два метода.

Предположим, что $\operatorname{dim} V \geqslant 4$ и $A^{2} V=\mathbb{Z} K_{V}^{2}$, где $A^{2} V$ есть группа классов циклов коразмерности два по модулю численной эквивалентности. Этому условию по теореме Лефшеца удовлетворяют, например, все гладкие полные пересечения Фано индекса 1 размерности $\geqslant 5$ в проективном пространстве (на самом деле, во взвешенном проективном пространстве; этот класс может быть еще расширен и включать семейства особых многообразий). Пусть $B \sim m K_{V}^{2}-$ неприводимое подмногообразие коразмерности два.

ПРЕДЛОЖЕНИЕ 2.9. Подмногообразие $B$ не может быть максималъным подмногообразием подвижной линейной системы $\Sigma \subset\left|-n K_{V}\right|$.

ДокАЗАТЕЛЬство. Предположим противное. Пусть $D_{1}, D_{2} \in \Sigma$ - общие дивизоры. Корректно определен эффективный цикл $Z=\left(D_{1} \circ D_{2}\right)$ теоретикосхемного пересечения $D_{1}$ и $D_{2}$, так как $\operatorname{codim}\left(D_{1} \cap D_{2}\right)=2$ в силу общности этих дивизоров. Цикл $Z$ (зависящий, конечно, от выбора $D_{1}, D_{2}$ ) назовем самопересечением системы $\Sigma$. Имеем

$$
n^{2} K_{V}^{2} \sim Z=\alpha B+Y
$$

где $Y$ - некоторый эффективный цикл коразмерности два и $\alpha>n^{2}$. Немедленно получаем противоречие. Предложение доказано.

Если $V$ - произвольное примитивное многообразие Фано (без каких-либо дополнительных предположений относительно $A^{2} V$ ), то приведенное рассуждение позволяет ограничить множество максимальных подмногообразий коразмерности два.

Точнее, пусть $Y \subset V$ - неприводимое подмногообразие (произвольной коразмерности). Назовем (антиканонической) степенью $Y$ число

$$
\operatorname{deg} Y=\left(Y \cdot\left(-K_{V}\right)^{\operatorname{dim} Y}\right) \in \mathbb{Z}_{+} .
$$

В частности, $\operatorname{deg} V=\left(-K_{V}\right)^{\operatorname{dim} V}$ есть степень самого многообразия $V$. В обозначениях доказательства предложения 2.9 имеем $\operatorname{deg} Z=n^{2} \operatorname{deg} V \geqslant \alpha \operatorname{deg} B$, откуда получаем оценку

$$
\operatorname{deg} B<\operatorname{deg} V
$$

Например, на трехмерной квартике $V_{4} \subset \mathbb{P}^{4}$ степень максимальной кривой может принимать значения 1, 2 и 3; как показано в [3], ни одна из этих возможностей не реализуется. Однако главный смысл оценки (15) в том, что класс 
подмногообразий коразмерности 2, которые могут быть максимальными, становится обозримым и возникающие возможности можно перебрать вручную.

Наиболее мощный метод исключения максимальных подмногообразий - это метод конусов, предложенный в [62], [64] и затем развивавшийся в [69], [75]. Опишем его на примере гладкой гиперповерхности Фано $V=V_{M} \subset \mathbb{P}^{M}$.

ЛЕмма 2.1. Для любой кривой $C \subset V$

$$
\operatorname{mult}_{C}|\chi| \leqslant n \text {. }
$$

ДокАЗАТЕЛЬСтво. Воспользуемся следующей конструкцией.

ПреДЛОЖенИЕ 2.10. Пустъ $C \subset W$, где $W \subset \mathbb{P}^{M}$ - гладкая гиперповерхность. Пусть $x \in \mathbb{P}^{M} \backslash W-$ точка общего положения, $C(x) \subset \mathbb{P}^{M}$ - конус с верииной $x$ и базой $C$. Тогда

$$
C(x) \cap W=C \cup R(x),
$$

где вычетная кривая $R(x)$ пересекает $C$ в $\operatorname{deg} R(x)$ различных точках.

ДокАЗАТЕЛЬСтво. Несложно проверить, что на поверхности $C(x)$ кривая $C$ численно эквивалентна гиперплоскому сечению. Нужно, однако, показать, что для общей точки $x$ все точки пересечения $R$ и $C$ имеют кратность 1 .

Очевидно, $C$ пересекает $R(x)$ в тех и только тех точках, где $C$ пересекает дивизор ветвления $W_{x} \subset W$ проекции $\pi: W \rightarrow \mathbb{P}^{M-1}$ из точки $x$. Гиперповерхность $W_{x}$ высекается на $W$ гиперповерхностью

$$
F_{x}=\sum_{i=0}^{M} \frac{\partial F}{\partial z_{i}} x_{i}=0
$$

где $\left(z_{0}: \ldots: z_{M}\right)$ - однородные координаты на $\mathbb{P}^{M}, F\left(z_{0}, \ldots, z_{M}\right)$ - уравнение $W$ относительно этих координат и $\left(x_{0}: \ldots: x_{M}\right)$ есть точка $x$. Но многообразие $W$ неособо, так что линейная система

$$
\left|\sum_{i=0}^{M} \lambda_{i} \frac{\partial F}{\partial z_{i}}\right|
$$

не имеет базисных точек. Поэтому для общей точки $x$ пересечение $C \cap W_{x}$ состоит из $(\operatorname{deg} W-1) \operatorname{deg} C=\operatorname{deg} R(x)$ различных точек, что и требовалось доказать.

Вернемся к доказательству леммы 2.1. Пусть $a \in \mathbb{P} \backslash V$ - общая точка, $R(a)$ вычетная кривая пересечения конуса $C(a)$ и гиперповерхности $V$. Тогда ограничение линейной системы $|\chi|$ на $R(a)$ есть линейный ряд степени $n \cdot \operatorname{deg} R(a)$, имеющий $\operatorname{deg} R(a)$ неподвижных точек кратности mult $_{C}|\chi|$, что и доказывает лемму.

Метод конусов позволяет оценивать кратности подмногообразий более высокой коразмерности, не только дивизоров [69], а также исключать максимальные особенности на различных типах гиперповерхностей и полных пересечений Фано [75], [53]. 
Имеются и другие методы исключения максимальных подмногообразий положительной размерности [3], [16], [59]-[61], [65]. Все они, тем или иным способом, реализуют одну и ту же идею: найти на многообразии $V$ подвижное подмногообразие $S$ малой размерности, имеющее возможно большее пересечение с максимальным подмногообразием, и ограничить систему $\Sigma$ на $S$. Получается эффективный дивизор на $S$, имеющий, однако, "слишком большие" особенности, что и дает противоречие; конкретные примеры см. в перечисленных выше работах.

2.2. $4 n^{2}$-неравенство. Обратимся к бесконечно близкому случаю. Пусть $E \subset V^{+}$- максимальная особенность системы $\Sigma$, причем (в обозначениях п. 1.2, которыми мы пользуемся без специальных оговорок) $\operatorname{dim} B_{0}<\operatorname{dim} B_{N-1}$. $\mathrm{B}$ частности, $\operatorname{codim} B_{0} \geqslant 3$. Положим $B=B_{0}$ и рассмотрим самопересечение $Z=\left(D_{1} \circ D_{2}\right)$ системы $\Sigma$. Напомним, что $n=c(\Sigma)>0$ есть порог канонического присоединения и выполнено неравенство Нётера-Фано (10) (в явной традиционной форме (13)).

Теорема 2.2 ( $4 n^{2}$-неравенство). Имеет место оценка

$$
\operatorname{mult}_{B} Z>4 n^{2} \text {. }
$$

Прежде чем доказывать теорему 2.2, сделаем несколько замечаний. Из этой теоремы немедленно следует бирациональная сверхжесткость трехмерной квартики: линейная система $\Sigma$ высекается на $V=V_{4}$ гиперповерхностями степени $n$, так что $\operatorname{deg} Z=4 n^{2}$ в смысле обычной степени в проективном пространстве. Далее, $B=p \in V$ - некоторая точка, так что $Z$ - эффективная кривая степени $4 n^{2}$ в $\mathbb{P}^{4}$, имеющая точку кратности больше, чем $4 n^{2}$, чего, конечно, не может быть. Это исключает максимальную особенность и доказывает бирациональную сверхжесткость. Из приведенного рассуждения также следует, что бесконечно близких максимальных особенностей нет на многообразиях Фано степени $\leqslant 4$. Впервые $4 n^{2}$-неравенство было опубликовано в [62], однако и само неравенство, и метод его доказательства извлечены из [3] (с существенными упрощениями), где они содержатся в неявной форме.

ДОКАЗАТЕЛЬСТво ТЕОРЕмы 2.2. Рассмотрим сначала следующую общую ситуацию.

Пусть $B \subset X, B \not \subset \operatorname{Sing} X,-$ неприводимое подмногообразие коразмерности $\geqslant 2, \sigma_{B}: X(B) \rightarrow X-$ его раздутие, $E(B)=\sigma_{B}^{-1}(B)-$ исключительный дивизор. Пусть $Z=\sum m_{i} Z_{i}, Z_{i} \subset E(B)$, - цикл размерности $k, k \geqslant \operatorname{dim} B$. Определим степень цикла $Z$, полагая

$$
\operatorname{deg} Z=\sum_{i} m_{i} \operatorname{deg}\left(Z_{i} \cap \sigma_{B}^{-1}(b)\right),
$$

где $b \in B$ - точка общего положения, $\sigma_{B}^{-1}(b) \cong \mathbb{P}^{\operatorname{codim} B-1}$ и степень справа есть обычная степень в проективном пространстве.

Отметим, что $\operatorname{deg} Z_{i}=0$ тогда и только тогда, когда $\sigma_{B}\left(Z_{i}\right)$ - собственное замкнутое подмножество подмногообразия $B$.

Наши вычисления основаны на следующем утверждении. 
Пусть $D$ и $Q$ - два различных простых дивизора Вейля на $X, D^{B}$ и $Q^{B}$ - их собственные прообразы на $X(B)$. Вообще, для любого подмногообразия $Y \subset X$ его собственный прообраз на $X(B)$ обозначаем символом $Y^{B}$.

Лемма 2.2. (i) Предположим, что $\operatorname{codim} B \geqslant 3$. Тогда

$$
D^{B} \circ Q^{B}=(D \circ Q)^{B}+Z
$$

где о обозначает иикл теоретико-схемного пересечения, $\operatorname{supp} Z \subset E(B), u$

$$
\operatorname{mult}_{B}(D \circ Q)=\left(\operatorname{mult}_{B} D\right)\left(\operatorname{mult}_{B} Q\right)+\operatorname{deg} Z \text {. }
$$

(ii) Предположим, что $\operatorname{codim} B=2$. Тогда

$$
D^{B} \circ Q^{B}=Z+Z_{1},
$$

где $\operatorname{supp} Z \subset E(B), \operatorname{supp} \sigma_{B}\left(Z_{1}\right)$ не содержит $B, u$

$$
D \circ Q=\left[\left(\operatorname{mult}_{B} D\right)\left(\operatorname{mult}_{B} Q\right)+\operatorname{deg} Z\right] B+\left(\sigma_{B}\right)_{*} Z_{1} .
$$

ДокАЗАТЕЛЬСтво легко получается применением стандартной теории пересечений [82].

Вернемся теперь к дискретному нормированию $\nu$.

Разобьем разрешение $\varphi_{i, i-1}: X_{i} \rightarrow X_{i-1}$ на нижнюю часть, $i=1, \ldots, L \leqslant K$, соответствующую раздутиям, для которых $\operatorname{codim} B_{i-1} \geqslant 3$, и верхнюю часть, $i=L+1, \ldots, K$, соответствующую раздутиям, для которых $\operatorname{codim} B_{i-1}=2$. Может случиться, что $L=K$ и верхняя часть пуста.

Пусть $D_{1}, D_{2} \in \Sigma$ - два различных общих дивизора. Определим последовательность циклов коразмерности два на многообразиях $X_{i}$, полагая

$$
\begin{aligned}
& D_{1} \circ D_{2}=Z_{0}, \\
& D_{1}^{1} \circ D_{2}^{1}=Z_{0}^{1}+Z_{1}, \\
& \ldots \ldots \ldots \ldots \ldots \ldots \ldots \ldots \ldots \ldots \ldots \ldots \\
& D_{1}^{i} \circ D_{2}^{i}=\left(D_{1}^{i-1} \circ D_{2}^{i-1}\right)^{i}+Z_{i},
\end{aligned}
$$

где $Z_{i} \subset E_{i}$. Тем самым для любого $i \leqslant L$ получаем:

$$
D_{1}^{i} \circ D_{2}^{i}=Z_{0}^{i}+Z_{1}^{i}+\cdots+Z_{i-1}^{i}+Z_{i}
$$

Для любых $j>i, j \leqslant L$ положим

$$
m_{i, j}=\operatorname{mult}_{B_{j-1}}\left(Z_{i}^{j-1}\right)
$$

(кратность неприводимого подмногообразия вдоль меньшего подмногообразия понимается в обычном смысле; для произвольного цикла продолжаем кратность по линейности).

Положим $d_{i}=\operatorname{deg} Z_{i}$. 
Получаем следующую систему равенств:

$$
\begin{aligned}
& \nu_{1}^{2}+d_{1}=m_{0,1}, \\
& \nu_{2}^{2}+d_{2}=m_{0,2}+m_{1,2}, \\
& \text {........................... } \\
& \nu_{i}^{2}+d_{i}=m_{0, i}+\cdots+m_{i-1, i}, \\
& \text {. } \\
& \nu_{L}^{2}+d_{L}=m_{0, L}+\cdots+m_{L-1, L} .
\end{aligned}
$$

Теперь

$$
d_{L} \geqslant \sum_{i=L+1}^{K} \nu_{i}^{2} \operatorname{deg}\left(\varphi_{i-1, L}\right)_{*} B_{i-1} \geqslant \sum_{i=L+1}^{K} \nu_{i}^{2} .
$$

ОПРЕДЕЛЕНИЕ 2.6. Функция $a:\{1, \ldots, L\} \rightarrow \mathbb{R}_{+}$называется согласованной со структурой графа, если

$$
a(i) \geqslant \sum_{j \rightarrow i} a(j)
$$

для любого $i=1, \ldots, L$.

В действительности будет использоваться только одна функция, согласованная со структурой графа, а именно $a(i)=p_{K i}=p_{i}$.

ПРЕДЛОЖЕНИЕ 2.11. Пусть $а(\cdot)$ - функиия, согласованная со структурой графа. Тогда имеет место неравенство

$$
\sum_{i=1}^{L} a(i) m_{0, i} \geqslant \sum_{i=1}^{L} a(i) \nu_{i}^{2}+a(L) \sum_{i=L+1}^{K} \nu_{i}^{2} .
$$

ДокАзАтЕльство. Докажем сначала некоторые вспомогательные утверждения.

ЛЕмма 2.3. Если $m_{i, j}>0$, mo $i \rightarrow j$.

ДокАЗАтЕльство. Если $m_{i, j}>0$, то некоторая компонента цикла $Z_{i}^{j-1}$ содержит $B_{j-1}$. Но $Z_{i}^{j-1} \subset E_{i}^{j-1}$. Что и требовалось доказать.

А теперь сравним кратности $m_{i j}$ с соответствующими степенями.

Лемма 2.4. Для любих $i \geqslant 1 u j \leqslant L$ имеем $m_{i, j} \leqslant d_{i}$.

ДокАЗАТЕЛЬСТВо. Подмногообразия $B_{a}$ неособы в своих общих точках. Но, поскольку отображения $\varphi_{a, b}: B_{a} \rightarrow B_{b}$ сюръективны, можно вычислять кратности в общих точках. Учитывая, что кратности не возрастают при раздутии неособого подмногообразия, сводим утверждение к очевидному случаю гиперповерхности в проективном пространстве. Лемма доказана.

В результате получаем следующую оценку:

$$
\sum_{j \geqslant i+1} a(j) m_{i, j}=\sum_{\substack{j \geqslant i+1 \\ m_{i, j} \neq 0}} a(j) m_{i, j} \leqslant d_{i} \sum_{j \rightarrow i} a(j) \leqslant a(i) d_{i} .
$$


Умножим $i$-е равенство в $(17)$ на $a(i)$ и сложим их все вместе: справа для любого $i \geqslant 1$ получим выражение

$$
\sum_{j \geqslant i+1} a(j) m_{i, j}
$$

В левой части для любого $i \geqslant 1$ получаем слагаемое $a(i) d_{i}$.

В силу сказанного выше, можно выбросить справа все слагаемые $m_{i, *}, i \geqslant 1$, а слева - все слагаемые $d_{i}, i \geqslant 1$, заменяя при этом знак равенства $=$ на знак неравенства $\leqslant$. Предложение 2.11 доказано.

СлеДСтвиЕ 2.2. Положим $m=m_{0,1}=\operatorname{mult}_{B}\left(D_{1} \circ D_{2}\right), D_{i} \in \Sigma$. Тогда имеет место неравенство

$$
m\left(\sum_{i=1}^{L} a(i)\right) \geqslant \sum_{i=1}^{L} a(i) \nu_{i}^{2}+a(L) \sum_{i=L+1}^{K} \nu_{i}^{2} .
$$

СледСтвиЕ 2.3. Имеет место следующее неравенство:

$$
m\left(\sum_{i=1}^{L} p_{i}\right) \geqslant \sum_{i=1}^{K} p_{i} \nu_{i}^{2}
$$

ДокАЗАТЕЛЬСтво. При $i \geqslant L+1$ очевидным образом $p_{i} \leqslant p_{L}$. Что и требовалось доказать.

Принимая во внимание неравенство Нётера-Фано для нормирования $\nu$, видим, что правая часть в этом неравенстве строго больше, чем значение квадратичной формы $\sum_{i=1}^{K} p_{i} \nu_{i}^{2}$ в точке

$$
\nu_{1}=\cdots=\nu_{K}=\frac{\sum_{i=1}^{K} p_{i} \delta_{i} n}{\sum_{i=1}^{K} p_{i}} .
$$

Теперь положим

$$
\Sigma_{l}=\sum_{\delta_{j} \geqslant 2} p_{j}, \quad \Sigma_{u}=\sum_{\delta_{j}=1} p_{j} .
$$

В этих обозначениях имеем:

$$
\operatorname{mult}_{B} Z>\frac{\left(2 \Sigma_{l}+\Sigma_{u}\right)^{2}}{\Sigma_{l}\left(\Sigma_{l}+\Sigma_{u}\right)} n^{2} .
$$

Теперь легкие вычисления показывают, что правая часть не меньше, чем $4 n^{2}$. Доказательство теоремы 2.2 завершено.

2.3. Второе доказательство: принцип связности. Ввиду важности теоремы 2.2 дадим другое ее доказательство. Его идея предложена в [50], однако приводимый вариант использует только так называемый принцип связности Шокурова-Коллара [30], [31] для сведения теоремы 2.2 к ранее известному факту о графах раздутий на неособых поверхностях [61]. Достаточно рассмотреть случай, когда $B=o$ - точка (возьмем общий росток неособого подмногообразия размерности $\operatorname{codim} B$ в точке общего положения $o \in B$, трансверсальный к $B$, и ограничим систему $\Sigma$ на этот росток). 
Пусть $S \ni$ о - общий росток гладкой поверхности на многообразии $X$. Очевидно, что $\Lambda=\left.\Sigma\right|_{S}$ - росток линейной системы кривых на $S$, имеющий точку о изолированной базисной точкой. Поскольку пара $(X,(1 / n) \Sigma)$ не канонична в точке o (это просто переформулировка неравенства Нётера-Фано (10)), то в силу обращения присоединения (которое есть прямое следствие принципа связности Шокурова-Коллара [30], [31]) пара $(S,(1 / n) \Lambda)$ не логканонична в точке $о$. Другими словами, для некоторого бирационального морфизма $\varphi: \widetilde{S} \rightarrow S$ гладких поверхностей существует простой дивизор $E \subset \widetilde{S}$, удовлетворяющий логварианту неравенства Нётера-Фано:

$$
\nu_{E}(\Lambda)>n(a(E)+1),
$$

где $a(\cdot)$ - дискрепантность, $\nu_{E}(\cdot)$ - кратность общего дивизора системы относительно $E$. Пусть $D_{1}, D_{2} \in \Lambda$ - общие кривые, $Z=\left(D_{1} \circ D_{2}\right)$ - нульмерная подсхема на $S$. Можно считать, что ее носитель - точка $o$.

ПрЕДЛОЖЕНИЕ 2.12. Имеет место следующее неравенство:

$$
\operatorname{mult}_{o} Z(=\operatorname{deg} Z)>4 n^{2} .
$$

Поскольку наши рассмотрения локальны, $\operatorname{mult}_{o} Z=\operatorname{deg} Z$ - это просто степень нульмерной схемы $Z$. Поскольку $S \ni o-$ общий росток поверхности, отсюда немедленно вытекает утверждение теоремы 2.2 .

ДоКАЗАТЕЛЬСТво ПРЕДлОЖЕНИЯ 2.12. Мы приведем элементарное рассуждение, основанное на явных вычислениях. Исходное рассуждение Корти см. в [50]. Пусть

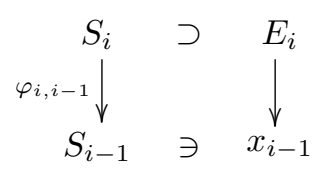

- разрешение дискретного нормирования $\nu_{E}, i=1, \ldots, N, x_{0}=o, x_{1}, \ldots, x_{N-1}-$ точки на $S_{1}, \ldots, S_{N-1}$ соответственно, где $x_{i} \in E_{i}$ и $\nu_{E_{N}}=\nu_{E}$. Пусть $\Gamma-$ граф этого разрешения: $\{1, \ldots, N\}$ - множество вершин, а вершины $i$ и $j, i>j$, соединены ориентированным ребром (обозначение: $i \rightarrow j$ всегда подразумевает, что $i>j$ ), если и только если $x_{i-1} \in E_{j}^{i-1}$, где $E_{j}^{i-1}-$ собственный прообраз исключительной прямой $E_{j}$ на $S_{i-1}$. Положим также:

$$
p_{j}=\text { (число путей из } N \text { в } j \text { ) }
$$

для $j \leqslant N-1, p_{N}=1$. Положим $\nu_{i}=\operatorname{mult}_{x_{i-1}} \Lambda^{i-1}$, где $\Lambda^{i-1}-$ собственный прообраз линейной системы $\Lambda$ на $S_{i-1}$. Легко видеть, что в терминах разрешения логнеравенство (18) принимает вид

$$
\sum_{i=1}^{N} p_{i} \nu_{i}>n\left(\sum_{i=1}^{N} p_{i}+1\right) .
$$

Кроме того, имеет место следующая оценка:

$$
\text { mult }_{o} Z \geqslant \sum_{i=1}^{N} \nu_{i}^{2} .
$$


Лемма 2.5. Справедливо следующее неравенство:

$$
\text { mult }_{o} Z>\frac{\left(\sum_{i=1}^{N} p_{i}+1\right)^{2}}{\sum_{i=1}^{N} p_{i}^{2}} n^{2} .
$$

ДокАЗАТЕЛьСтво получается простыми вычислениями: минимум квадратичной формы

$$
\sum_{i=1}^{N} s_{i}^{2}
$$

при ограничении

$$
\sum_{i=1}^{N} p_{i} s_{i}=c
$$

достигается при $s_{i}=p_{i} a$, где общая константа $a$ находится из равенства (19). Лемма доказана.

Ввиду этой леммы предложение 2.12 вытекает из следующего факта.

ЛЕмма 2.6. Справедливо следующее неравенство:

$$
\left(\sum_{i=1}^{N} p_{i}+1\right)^{2} \geqslant 4 \sum_{i=1}^{N} p_{i}^{2} .
$$

ДокАзАтельство. Отметим, прежде всего, что в неравенстве (20) равенство может достигаться: например, когда $N=1$. Считаем, что $N \geqslant 2$. Положим

$$
\{2, \ldots, k \leqslant N\}=\{i \mid i \rightarrow 1\} .
$$

По определению чисел $p_{i}$ получаем равенство

$$
p_{1}=\sum_{i \rightarrow 1} p_{i}=\sum_{i=2}^{k} p_{i}
$$

Следовательно, неравенство (20) может быть переписано в следующем виде:

$$
\left(2 p_{1}+\sum_{i=k+1}^{N} p_{i}+1\right)^{2} \geqslant 4 \sum_{i=1}^{N} p_{i}^{2}
$$

или, после несложного преобразования,

$$
4\left(\sum_{i=2}^{k} p_{i}\right)\left(\sum_{i=k+1}^{N} p_{i}+1\right)+\left(\sum_{i=k+1}^{N} p_{i}+1\right)^{2} \geqslant 4 \sum_{i=2}^{k} p_{i}^{2}+4 \sum_{i=k+1}^{N} p_{i}^{2} .
$$

Легко видеть, что если $k=N$, то подграф графа $\Gamma$ с вершинами $\{2, \ldots, N\}$ имеет вид $2 \leftarrow 3 \leftarrow \cdots \leftarrow N$ (поскольку на любой поверхности $S_{i}$ кривая $\bigcup_{j \leqslant i} E_{j}^{i}$ есть в силу гладкости дивизор с нормальными пересечениями). Значит, $p_{2}=\cdots=p_{N}=1$ и неравенство (20) выполнено очевидным образом. Поэтому 
будем считать, что $N \geqslant k+1$. Рассуждая по индукции по числу вершин графа $\Gamma$, можно предполагать, что неравенство

$$
\left(\sum_{i=k+1}^{N} p_{i}+1\right)^{2} \geqslant 4 \sum_{i=k+1}^{N} p_{i}^{2}
$$

справедливо. Следовательно, достаточно показать, что справедлива следующая оценка:

$$
\left(\sum_{i=2}^{k} p_{i}\right)\left(\sum_{i=k+1}^{N} p_{i}+1\right) \geqslant \sum_{i=2}^{k} p_{i}^{2}
$$

Если $k=2$, то по построению получаем:

$$
p_{2} \leqslant \sum_{i=3}^{N} p_{i}
$$

откуда немедленно вытекает неравенство (21). Если $k \geqslant 3$, то подграф графа Г с вершинами $\{2, \ldots, k\}$ есть цепь:

$$
2 \leftarrow 3 \leftarrow \cdots \leftarrow k .
$$

Поскольку $k \rightarrow(k-1)$ и $k \rightarrow 1$, вершины $k$ и $i, i \in\{2, \ldots, k-2\}$, не соединены стрелкой (ориентированным ребром). Следовательно, $j \nrightarrow i$ при $j \geqslant k+1$, $i \in\{2, \ldots, k-2\}$. Тем самым, каждый путь из вершины $N$ в вершину $i \in$ $\{2, \ldots, k-2\}$ должен проходить через вершину $k-1$. Поэтому

$$
p_{2}=\cdots=p_{k-1}=p_{k}+\sum_{\substack{i \rightarrow k-1 \\ i \geqslant k+1}} p_{i} .
$$

Лемма 2.7. Для любого $i \in\{1, \ldots, N\}$ справедливо следующее неравенство:

$$
p_{i} \leqslant \sum_{j \geqslant i+2} p_{j}+1
$$

(если множество $\{j \geqslant i+2\}$ пусто, сумма считается равной нулю).

ДокАзАТЕльство. Утверждение леммы - чисто комбинаторный факт, устанавливаемый убывающей индукцией по $i$. Оставляем его читателю в качестве упражнения (полное рассуждение см. в [70]).

Вернемся к доказательству леммы 2.6. Имеем

$$
p_{2}=\cdots=p_{k-1} \leqslant \sum_{i=k+1}^{N} p_{i}+1 .
$$

Но $p_{k} \leqslant p_{k-1}$, так что

$$
\left(\sum_{i=2}^{k} p_{i}\right)\left(\sum_{i=k+1}^{N} p_{i}+1\right) \geqslant p_{k-1} \sum_{i=2}^{k} p_{i} \geqslant \sum_{i=2}^{k} p_{i}^{2},
$$

что и требовалось. Лемма 2.6 доказана.

Принцип связности Шокурова-Коллара и его приложения к доказательству бирациональной жесткости будут подробно обсуждаться в последующем обзоре. 


\section{§ 3. Откручивание максимальных особенностей}

3.1. Откручивающие бирациональные автоморфизмы. Пусть $\Sigma$ подвижная линейная система на многообразии $V$, имеющая максимальную особенность $E$. Предположим, что (в терминологии и обозначениях $\S 1$ ) особенность $E$ можно открутить бирациональным автоморфизмом $\tau_{E} \in \operatorname{Bir} V$, зависящим толъко от особенности E. В силу этого предположения об универсальности можно рассчитывать на то, что

- линейная система $\left(\tau_{E}^{-1}\right)_{*}\left|-n K_{V}\right|$, задающая само отображение $\tau_{E}$, имеет максимальную особенность $E$;

- автоморфизм $\tau_{E}$ откручивает особенность $E$ у самого себя, так что композиция $\tau_{E}^{2}$ не имеет $E$ максимальной особенностью; более того, естественно предположить, что $\tau_{E}^{2}$ не имеет максимальных особенностей вообще.

Во всех изученных случаях именно так и происходит. Все найденные откручивающие преобразования оказываются инволюциями: $\tau_{E}^{2}=\mathrm{id}$. Однако это чисто эмпирический факт. Существует два способа строить откручивающие инволюции. Первый способ применяется тогда, когда максимальная особенность $E$ порождает рациональное отображение $V \stackrel{2: 1}{2-\rightarrow} W$ степени 2 . В этом случае $\tau_{E} \in \operatorname{Bir} V$ есть инволюция Галуа, переставляющая точки в общем слое. Образ особенности $E$ относительно $\tau_{E}$ есть дивизор на $V$. Второй способ применяется тогда, когда особенность $E$ порождает рациональное отображение $V \stackrel{\gamma}{-\rightarrow} W$, общий слой которого - эллиптическая кривая, причем $\gamma$ имеет (рациональное) сечение. Эллиптическая кривая $C$ с фиксированной точкой $o \in C$ есть абелево многообразие, $C \cong \mathbb{C} / \Lambda$, где точка о соответствует $0 \in \mathbb{C}$, что позволяет определить (эллиптическое) отражение $z \mapsto-z$ (в смысле указанного изоморфизма). Теперь $\tau_{E} \in \operatorname{Bir} V$ есть $\gamma$-послойное отображение, на общем слое $\gamma^{-1}(w)$ действующее как эллиптическое отражение.

Простейший (и, вероятно, исторически самый первый) пример инволюции Галуа дает откручивание точки на кубической гиперповерхности $V=V_{3} \subset$ $\mathbb{P}^{M+1}, M \geqslant 3$. Пусть $о \in V-$ некоторая точка, $\varphi: \widetilde{V} \rightarrow V-$ ее раздутие, $E=\varphi^{-1}(o) \subset \widetilde{V}-$ исключительный дивизор. Имеем

$$
\operatorname{Pic} V=\mathbb{Z} H, \quad \operatorname{Pic} \widetilde{V}=\mathbb{Z} H \oplus \mathbb{Z} E, \quad K_{\widetilde{V}}=-(M-1) H+(M-1) E,
$$

где $H \in \operatorname{Pic} V$ - класс гиперплоского сечения. Дискрепантность $a(E)$ равна $M-1$, так что подвижная система $\Sigma \subset|n H|$ имеет $E$ максимальной особенностью, если выполнено неравенство

$$
\nu=\operatorname{mult}_{o} \Sigma>n
$$

(для собственного прообраза $\widetilde{\Sigma}$ на $\widetilde{V}$ имеем $\widetilde{\Sigma} \subset|n H-\nu E|)$. Существуют ли такие линейные системы?

Пусть $\left(z_{1}, \ldots, z_{M+1}\right)$ - некоторая система аффинных координат с началом в точке $o$,

$$
f=q_{1}+q_{2}+q_{3}, \quad \operatorname{deg} q_{i}=i,
$$


- разложение Тейлора уравнения $f\left(z_{*}\right)$ гиперповерхности $V$. Очевидно, что замкнутое множество $Y \subset V$, состоящее из всех прямых на $V$, проходящих через точку $о$, задается системой уравнений $q_{1}=q_{2}=q_{3}=0$.

ПрЕДЛОжЕНИЕ 2.13. Подвижная линейная система, удовлетворяющая неравенству (24), существует тогда и только тогда, когда $\operatorname{codim}_{V} Y=2$.

ДОКАЗАТЕЛЬСтво. Для любой прямой $L \subset \mathbb{P}^{M+1}, o \in L \subset V$, и произвольного дивизора $D \in \Sigma$ имеем $(D \cdot L)=n$, так что из неравенства $\operatorname{mult}_{o} D>n$ следует, что $L \subset D$. Поэтому если $\operatorname{codim}_{V} Y=1$, то линейная система $\Sigma$ не может быть подвижной. Обратно, если $\operatorname{codim}_{V} Y=2$, то рассмотрим линейную систему $\Lambda$, высекаемую на $V$ квадриками

$$
\lambda\left(q_{1}+q_{2}\right)+l_{1}\left(z_{*}\right) q_{1},
$$

где $\lambda \in \mathbb{C}, l_{1}\left(z_{*}\right)$ - произвольная линейная форма. Очевидно, что в силу конструкции

$$
\left.\left(q_{1}+q_{2}\right)\right|_{V}=-\left.q_{3}\right|_{V},\left.\quad q_{1}\right|_{V}=-\left.\left(q_{2}+q_{3}\right)\right|_{V},
$$

так что mult $_{o} \Lambda \geqslant 3$. Поскольку $\Lambda \subset|2 H|$ и базисное множество системы $\Lambda$ есть в точности $Y$, система $\Lambda$ не имеет неподвижных компонент. Предложение доказано.

Очевидно, что для общей точки $o \in V$ множество $Y$ имеет коразмерность 2 . Предполагая это, зафиксируем некоторую подвижную систему $\Sigma$ с максимальной особенностью $E$. Рассмотрим проекцию $\pi_{o}: \mathbb{P}^{M+1} \rightarrow \mathbb{P}^{M}$ из точки $о$ и ограничим ее на $V$. Очевидно, что $\pi=\left.\pi_{o}\right|_{V}: V \rightarrow \mathbb{P}^{M}$ есть рациональное отображение степени 2, так что имеется инволюция Галуа $\tau_{E} \in \operatorname{Bir} V$. Пусть $\widetilde{Y} \subset \widetilde{V}-$ собственный прообраз конуса $Y$ на $\widetilde{V}$.

ПРЕДЛОЖЕНИЕ 2.14. Инволющия $\tau_{E}$ продолжается до бирегулярного автоморфизма открытого $\tau_{E}$-инвариантного множества $\widetilde{V} \backslash \widetilde{Y}=U, \tau_{E} \in \operatorname{Aut} U$.

ДокАЗАТЕЛЬСтво. Это очевидно.

Поскольку $\widetilde{Y} \subset \widetilde{V}$ - замкнутое множество коразмерности два, действие инволюции $\tau_{E}$ на группе Пикара $\operatorname{Pic} \widetilde{V}$ корректно определено.

ПреДЛОЖениЕ 2.15. Действие $\tau_{E}$ на групnе $\operatorname{Pic} \widetilde{V}=\mathbb{Z} H \oplus \mathbb{Z} E$ задается формулами

$$
\tau_{E}^{*} H=2 H-3 E, \quad \tau_{E}^{*}=H-2 E .
$$

ДокАЗАТЕЛЬство. Это очевидно в силу $\tau_{E}$-инвариантности класса $H-E$ и равенства $\tau_{E}(E)=V \cap T_{o} V$, где $T_{o} V \subset \mathbb{P}^{M+1}$ - касательная гиперплоскость в точке $о$. Предложение доказано.

Пусть, наконец, $\Sigma_{1}=\left(\tau_{E}\right)_{*} \Sigma-$ собственный прообраз линейной системы $\Sigma$ относительно $\tau_{E}, \widetilde{\Sigma}_{1}-$ собственный прообраз системы $\Sigma_{1}$ на $\widetilde{V}$. Имеем, очевидно,

$$
\widetilde{\Sigma}_{1}=\tau_{E}(\widetilde{\Sigma}) \subset\left|n \tau_{E}^{*}-\nu \tau_{E}^{*} E\right|=\left|n_{1} H-\nu_{1} E\right|,
$$

где, согласно предложению $2.15, n_{1}=2 n-\nu$ и $\nu_{1}=3 n-2 \nu$. 
Поскольку по предположению $\nu>n$, для порогов канонического присоединения получаем неравенство

$$
c\left(\Sigma_{1}\right)=n_{1}=2 n-\nu<n=c(\Sigma) .
$$

Кроме того, $n_{1}-\nu_{1}=\nu-n>0$, так что $E$ уже не является максимальной особенностью системы $\Sigma_{1}$. Процедура откручивания завершена.

Второй метод построения откручивающих отображений (с помощью эллиптических расслоений) вполне аналогичен первому, хотя с вычислительной точки зрения обычно более сложен. Отметим, что если эллиптическая кривая $C$ вложена в $\mathbb{P}^{2}$ в качестве гладкой кубической кривой, то эллиптическая инволюция относительно точки $o \in C \subset \mathbb{P}^{2}$ (принятой в качестве нуля группового закона на $C$ ) есть проективное отражение кубической кривой $C \subset \mathbb{P}^{2}$ относительно точки $p \in C$, однозначно определенной условием

$$
\mathscr{O}_{C}(2 o+p)=\left.\mathscr{O}_{\mathbb{P}^{1}}(1)\right|_{C},
$$

так что эллиптические инволюции легко сводятся к проективным отражениям. Однако явные геометрические конструкции инволюций типа Галуа и эллиптических инволюций существенно различаются, как будет видно в пп. $3.2,3.3$ на примере многообразия $V_{2 \cdot 3} \subset \mathbb{P}^{5}$. Этот пример иллюстрирует и появление соотношений между откручивающими инволюциями, когда процедура откручивания определена неоднозначно.

Техника откручивания максимальных особенностей, включая описание соотношений между образующими группы бирациональных автоморфизмов, приняла современный вид в работах Ю. И. Манина о поверхностях над незамкнутыми полями [12], [13], [41]. Известно не так много многообразий размерности 3 и выше (среди полностью изученных), имеющих нетривиальную группу бирациональных автоморфизмов, т. е. требующих реального применения процедуры откручивания. Вот основные типы таких многообразий Фано:

- двойные квадрики индекса $1 V_{4} \stackrel{2: 1}{\longrightarrow} Q_{2} \subset \mathbb{P}^{4}[16]$;

- полное пересечение квадрики и кубики $V_{2 \cdot 3} \subset \mathbb{P}^{5}$ [16], [78], рассмотренное ниже;

- трехмерная квартика с двойной точкой $V_{4} \subset \mathbb{P}^{4}[61]$ (случай нескольких двойных точек рассмотрен в [81]);

- 95 семейств взвешенных гиперповерхностей Фано [83];

- гиперповерхности Фано $V_{M} \subset \mathbb{P}^{M}, M \geqslant 5$, с особой точкой кратности $M-2$ общего положения [68] (единственный изученный пример в произвольной размерности).

Кроме того, нетривиальная процедура откручивания применялась к некоторым семействам расслоений Фано: пучкам поверхностей дель Пеццо [40], [49], [84], [85] и пучкам многомерных многообразий Фано индекса 1 [72], [75], [76]. Однако относительный случай выходит за рамки настоящего обзора и будет рассмотрен во второй части работы, посвященной расслоениям над нетривиальной базой.

3.2. Полное пересечение квадрики и кубики: образующие. Мы изучаем (следуя [78]) полное пересечение $V=Q \cap F \subset \mathbb{P}^{5}$, где $Q$ - квадрика и $F$ - кубическая гиперповерхность. Многообразие $V$ предполагается гладким 
и, более того, общим в смысле, определенном ниже, в частности, $\mathrm{Pic} V=\mathbb{Z} H$, где $H=-K_{V}$ класс гиперплоского сечения $V \subset \mathbb{P}^{5}$.

Пусть $L \subset V-$ прямая в $\mathbb{P}^{5}$.

ПРеДЛОЖЕНИЕ 2.16. Для нормалъного пучка $\mathscr{N}_{L / V}$ имеются две возможности:

- либо $\mathscr{N}_{L / V} \cong \mathscr{O}_{L}(-1) \oplus \mathscr{O}_{L}(L-$ прямая общего типа);

- либо $\mathscr{N}_{L / V} \cong \mathscr{O}_{L}(-2) \oplus \mathscr{O}_{L}(1)(L-$ прямая необщего типа).

Более того, прямая $L$ является прямой необщего типа, если и только если выполнено любое из двух эквивалентных условий:

- существует плоскость $P \subset \mathbb{P}^{5}$ такая, ито $L \subset P$ и теоретико-схемное пересечение $V \cap P$ неприведено в каждой точке прямой $L$;

- пусть $\sigma: \widetilde{V} \rightarrow V$ - раздутие $L, E=\sigma^{-1}(L)$ - исключительный дивизор, тогда, ограничивая на $E$ собственный прообраз на $\widetilde{V}$ общего гиперплоского сечения, содержащего $L$, получаем необильный дивизор на $E$.

\section{ДоКАЗАТЕЛЬСТВО несложно и оставляется читателю.}

Будем рассматривать общее полное пересечение $V=Q \cap F$, удовлетворяющее следующим условиям:

- $V$ не содержит прямых необщего типа (обычным подсчетом размерностей легко проверяется, что данное условие выполнено для общего полного пересечения);

- на $V$ нет тройки прямых, лежащих в одной плоскости и имеющих общую точку;

- квадрика $Q$ невырожденная.

Пусть $L \subset V-$ некоторая прямая. Проекция $\mathbb{P}^{5} \rightarrow \mathbb{P}^{3}$ из $L$ определяет рациональное отображение $\pi_{L}: V \rightarrow \mathbb{P}^{3}$ степени два. Пусть $\alpha_{L} \in \operatorname{Bir} V-$ соответствующая инволюция Галуа.

Более формально, пусть $\sigma: \widetilde{V} \rightarrow V$ - раздутие $L, E=\sigma^{-1}(L) \subset \widetilde{V}$ - исключительный дивизор. Отображение $\pi_{L}$ продолжается до морфизма $p=\pi_{L} \circ \sigma$ : $\widetilde{V} \rightarrow \mathbb{P}^{3}$.

ЛЕмма 2.8. Морфизм р есть конечный морфизм степени два вне замкнутого подмножества $W \subset \widetilde{V}$ коразмерности два и $p(W) \subset \mathbb{P}^{3}$ есть конечное множество точек. Инволючия $\alpha_{L}$ продолжается до бирегулярной инволючии множества $\widetilde{V} \backslash W$. Ее действие на $\operatorname{Pic} \widetilde{V}=\mathbb{Z} H \oplus \mathbb{Z} E$ дается формулами

$$
\alpha_{L}^{*}(H)=4 H-5 E, \quad \alpha_{L}^{*}(E)=3 H-4 E .
$$

ДокАЗАТЕЛЬство. Проекция $p: \widetilde{V} \rightarrow \mathbb{P}^{3}$ есть конечный морфизм вне множества $W \subset \widetilde{V}$, состоящего из кривых, стягиваемых морфизмом $p$. Покажем, что таких кривых конечное число. Пусть $H^{\prime}=n H-\nu E$ и $E^{\prime}=m H-\mu E-$ классы в $\mathrm{Pic} \widetilde{V}$ собственного прообраза общего гиперплоского сечения и дивизора $E$ относительно $\alpha_{L}$. Линейная система $|H-E|$ очевидным образом инвариантна относительно $\alpha_{L}$. Возьмем общую поверхность $S \in|H-E|$. 
Поскольку $K_{S}=0$, бирациональная инволюция $\left.\alpha_{L}\right|_{S}$ продолжается до бирегулярной инволюции этой поверхности. Обозначим ее символом $\alpha_{S}$, а ограничения $H$ и $E$ на $S$ символами $H_{S}$ и $E_{S}$ соответственно. Получаем

$$
\alpha_{S}^{*} H_{S}=n H_{S}-\nu E_{S}, \quad \alpha_{S}^{*} E_{S}=m H_{S}-\mu E_{S}
$$

и класс $H_{S}-E_{S} \alpha_{S}^{*}$-инвариантен, откуда имеем $n=m+1, \nu=\mu+1$. Поскольку $\alpha_{S}$ есть автоморфизм, то

$$
\left(\alpha_{S}^{*} H_{S} \cdot\left(H_{S}-E_{S}\right)\right)=\left(H_{S} \cdot\left(H_{S}-E_{S}\right)\right)=5
$$

и $\left(\alpha_{S}^{*} H_{S}\right)^{2}=\left(H_{S}\right)^{2}=6$, откуда в силу очевидных равенств $\left(H_{S} \cdot E_{S}\right)=1$, $E_{S}^{2}=-2$ получаем следующие две возможности для $n, m, \nu, \mu$ :

- либо $H^{\prime}=4 H-5 E, E^{\prime}=3 H-4 E$;

- либо $H^{\prime}=H, E^{\prime}=E$;

последнее очевидным образом невозможно, поскольку $\alpha_{L}$ не может быть продолжено до бирегулярного автоморфизма $V$.

По построению система $|4 H-5 E|$ подвижна. Однако если кривая $C$ стягивается морфизмом $p$, то $(C \cdot(H-E))=0$ и поэтому $\left(C \cdot H^{\prime}\right)<0$. Мы заключаем, что таких кривых может быть лишь конечное число. Лемма 2.8 доказана.

Теперь пусть $P \subset \mathbb{P}^{5}-2$-плоскость такая, что $P \cap V$ - объединение трех прямых, $P \cap V=L \cup L_{1} \cup L_{2}$. Такое возможно, только если $P \subset Q$. Пусть $\sigma: \widetilde{V} \rightarrow V-$ композиция трех раздутий: сначала раздуваем $L$, затем собственный прообраз $L_{1}$ и наконец собственный прообраз $L_{2}$.

Исключительные дивизоры на $\widetilde{V}$, отвечающие прямым $L, L_{1}, L_{2}$, обозначаем символами $E, E_{1}, E_{2}$ соответственно.

ЛЕмма 2.9. Инволюция $\alpha_{L}$ продолжается до бирегулярной инволюиии на $\widetilde{V} \backslash W$, где $W$ - замкнутое подмножество коразмерности два. Действие $\alpha_{L}$ на $\operatorname{Pic} \widetilde{V}=\mathbb{Z} H \oplus \mathbb{Z} E \oplus \mathbb{Z} E_{1} \oplus \mathbb{Z} E_{2}$ дается формулами

$$
\begin{aligned}
\alpha_{L}^{*} & =4 H-5 E-2 E_{1}-2 E_{2}, \\
\alpha_{L}^{*} E & =3 H-4 E-2 E_{1}-2 E_{2}, \\
\alpha_{L}^{*} E_{i} & =E_{j},
\end{aligned}
$$

əде $\{i, j\}=\{1,2\}$.

ДокАЗАТЕЛЬСТво аналогично предыдущему.

Рассмотрим теперь коники на многообразии $V$.

Легко видеть, что имеется одномерное семейство неприводимых коник $Y \subset V$ таких, что плоскость $P(Y)=\langle Y\rangle$ целиком содержится в квадрике $Q$. Очевидно,

$$
P(Y) \cap V=Y \cup L(Y)
$$

где $L(Y)$ - вычетная прямая. Описанные коники будем называть специальнъми кониками.

Каждая специальная коника $Y$ порождает следующую конструкцию. Положим $P=P(Y)$. Рассмотрим проекцию $\pi_{P}: \mathbb{P}^{5} \rightarrow-\rightarrow \mathbb{P}^{2}$ из плоскости $P$. Слои $\pi_{P}$ 
суть 3-плоскости $S \supset P$, так что $S \cap Q=P \cup P(S)$, где $P(S)$ - вычетная плоскость. Следовательно, $\pi_{P}$ расслаивает $V$ над $\mathbb{P}^{2}$ на эллиптические кривые $C_{S}=P(S) \cap F-$ плоские кубики. Общая кривая $C_{S}$ пересекает вычетную прямую $L(Y)$ в одной точке, которая есть пересечение $L(Y) \cap P(S)$. Определим инволюцию $\beta_{Y} \in \operatorname{Bir} V$ как послойное отображение, полагая $\left.\beta_{Y}\right|_{C_{S}}$ эллиптическим отражением, где групповой закон на $C_{S}$ задается точкой $L(Y) \cap P(S)$ в качестве нуля.

Пусть $\sigma: \widetilde{V} \rightarrow V$ - композиция раздутия коники $Y$ и раздутия собственного прообраза прямой $L(Y), E$ и $E^{+}$- соответствующие исключительные дивизоры. Очевидно, что $\pi_{P} \circ \sigma: V \rightarrow \mathbb{P}^{2}-$ морфизм, общий слой которого есть эллиптическая кривая $C_{t}, t \in \mathbb{P}^{2}$. Дивизор $E^{+}$есть сечение этого эллиптического расслоения, $\left(E^{+} \cdot C_{t}\right)=1$.

ЛЕмма 2.10. Бирациональная инволючия $\beta_{Y}$ продолжается до бирегулярной инволюиии на дополнении $\widetilde{V} \backslash W$, где $W$ есть замкнутое подмножество коразмерности два и, более того, $\pi_{P} \circ \sigma(W) \subset \mathbb{P}^{2}$ есть конечное множество. Действие $\beta_{Y}$ на $\operatorname{Pic} \widetilde{V}=\mathbb{Z} H \oplus \mathbb{Z} E \oplus \mathbb{Z} E^{+}$дается формулами

$$
\begin{aligned}
\beta_{Y}^{*} H & =13 H-14 E-8 E^{+}, \\
\beta_{Y}^{*} E & =12 H-13 E-8 E^{+}, \\
\beta_{Y}^{*} E^{+} & =E^{+} .
\end{aligned}
$$

ДокАЗАТЕЛЬСтво аналогично доказательству леммы 2.8 .

Как будет показано в следующем пункте, инволюции $\alpha_{L}$ и $\beta_{Y}$ позволяют открутить максимальные особенности любой подвижной системы на многообразии $V$.

3.3. Полное пересечение квадрики и кубики: соотношения. Пусть $P \subset \mathbb{P}^{5}$ - такая плоскость, что $P \subset Q$ и $P \cap F=L_{1} \cup L_{2} \cup L_{3}$ - объединение трех прямых.

Лемма 2.11. Имеет место следующее соотношение:

$$
\left(\alpha_{L_{1}} \circ \alpha_{L_{2}} \circ \alpha_{L_{3}}\right)^{2}=\mathrm{id}_{V} .
$$

ДоКАЗАТЕЛЬСтво. Очевидно, что каждая из трех инволюций $\alpha_{L_{i}}$ сохраняет слои проекции $\pi_{P}: V \rightarrow \mathbb{P}^{2}$ из плоскости $P$. Напомним, что общий слой $\pi_{P}^{-1}(t)$ есть кубическая кривая $C_{t}$, где $C_{t} \cap P=\left\{x_{1}, x_{2}, x_{3}\right\}, x_{i}=C_{t} \cap L_{i}$. Возьмем точку $x \in C_{t}$; очевидно, что

$$
\alpha_{L_{i}}(x)+x+x_{i} \sim x_{1}+x_{2}+x_{3}
$$

на $C_{t}$. Поэтому вычисляем:

$$
\begin{aligned}
\alpha_{L_{3}}(x) & \sim x_{1}+x_{2}-x, \\
\alpha_{L_{2}} \circ \alpha_{L_{3}}(x) & \sim x_{3}-x_{2}+x, \\
\alpha_{L_{1}} \circ \alpha_{L_{2}} \circ \alpha_{L_{3}}(x) & \sim 2 x_{2}-x, \\
\left(\alpha_{L_{1}} \circ \alpha_{L_{2}} \circ \alpha_{L_{3}}\right)^{2}(x) & \sim x,
\end{aligned}
$$

что и требовалось. 
После проделанной подготовительной работы мы можем сформулировать основную теорему, описывающую бирациональную геометрию многообразия $V$.

Пусть $\mathscr{L}$ и $\mathscr{C}$ - множества прямых и специальных коник на $V$ соответственно. Пусть $G^{+}-$свободная группа, порожденная символами $A_{L}$ и $B_{Y}$ для всех $L \in \mathscr{L}$ и $Y \in \mathscr{C}$ соответственно. Пусть $R^{+} \subset G^{+}-$нормальная подгруппа, порожденная словами $A_{L}^{2}$ для всех $L \in \mathscr{L}, B_{Y}^{2}$ для всех $Y \in \mathscr{C}$ и, наконец, $\left(A_{L_{1}} A_{L_{2}} A_{L_{3}}\right)^{2}$ для всех троек различных прямых $L_{1}, L_{2}, L_{3} \in \mathscr{L}$ таких, что $\left\langle L_{1} \cup L_{2} \cup L_{3}\right\rangle=\mathbb{P}^{2}$.

Пусть $G=G^{+} / R^{+}$- факторгруппа. Построим полупрямое произведение $G$ Aut $V$, используя очевидное действие группы Aut $V$ на $G$ : для $\rho \in \operatorname{Aut} V$ положим

$$
\rho A_{L} \rho^{-1}=A_{\rho(L)}, \quad \rho B_{Y} \rho^{-1}=B_{\rho(Y)} .
$$

Пусть $\varepsilon:$ Aut $V \rightarrow \operatorname{Bir} V$ - гомоморфизм, переводящий $A_{L}$ в $\alpha_{L}, B_{Y}$ в $\beta_{Y}$ и тождественный на Aut $V$.

Теорема 2.3 [78]. Многообразие $V$ бирационально жесткое и в есть изоморфизм групп.

ДоКАЗАТЕЛЬСТво. Положим $\mathscr{B}=\mathscr{L} \cup \mathscr{C}$. Рассмотрим произвольную подвижную линейную систему $\Sigma \subset|n H|$ на $V$. Очевидно, что $c(\Sigma, V)=n$. Чтобы доказать, что $\varepsilon$ есть биекция, в качестве $\Sigma$ возьмем собственный прообраз линейной системы $|H|$ гиперплоских сечений относительно фиксированного бирационального автоморфизма $\chi \in \operatorname{Bir} V$. Очевидно, что в этом случае $n=1$ тогда и только тогда, когда $\chi \in \operatorname{Aut} V$ (и по построению бирегулярные автоморфизмы содержатся в образе $\varepsilon$ ).

Бирациональную жесткость и сюръективность $\varepsilon$ будем доказывать одновременно, используя следующий ключевой технический факт.

ПРЕДЛОЖЕНИЕ 2.17. Предположим, что $c_{\text {virt }}(\Sigma)<n$. Тогда существует подмногообразие $B \in \mathscr{B}$ (т.е. прямая или специальная коника) такое, что $\operatorname{mult}_{B} \Sigma>n$. Более того, имеется, самое большее, два подмногообразия в $\mathscr{B}$ с этим свойством, и если их два, скажем, $B_{1}, B_{2} \in \mathscr{B}$, то они суть прямые, $B_{1}, B_{2} \in \mathscr{L}$, их линейная оболочка $\left\langle B_{1}, B_{2}\right\rangle$ есть плоскость $P=\mathbb{P}^{2}$ и $P \subset Q$.

ДокАЗАТЕЛЬСТВо см. в [78].

Лемма 2.12. (i) Пусть $L \subset V-$ прямая, $\Sigma^{+} \subset\left|n_{+} H\right|-$ собственный прообраз линейной системы $\Sigma$ относительно $\alpha_{L}$. Тогда выполнены следующие равенства:

$$
n_{+}=4 n-3 \operatorname{mult}_{L} \Sigma, \quad \operatorname{mult}_{L} \Sigma^{+}=5 n-4 \operatorname{mult}_{L} \Sigma .
$$

(ii) Пусть $Y \in \mathscr{C}$ - специальная коника, $L=L(Y) \in \mathscr{L}$ - вычетная прямая, $\Sigma^{+} \subset\left|n_{+} H\right|-$ собственный прообраз линейной системы $\Sigma$ относительно $\beta_{Y}$. Тогда выполнены следующие равенства:

$$
\begin{gathered}
n_{+}=13 n-12 \text { mult }_{Y} \Sigma, \quad \operatorname{mult}_{Y} \Sigma^{+}=14 n-13 \text { mult }_{Y} \Sigma, \\
\operatorname{mult}_{L} \Sigma^{+}=8 n-8 \text { mult }_{Y} \Sigma+\operatorname{mult}_{L} \Sigma .
\end{gathered}
$$


(iii) Пусть $P \subset \mathbb{P}^{5}-2$-плоскость такая, что $P \cap V=L \cup L_{1} \cup L_{2}, \Sigma^{+}$как в части (i) выше. Тогда для $\{i, j\}=\{1,2\}$ имеем

$$
\operatorname{mult}_{L_{i}} \Sigma^{+}=2 n-2 \text { mult }_{L} \Sigma+\operatorname{mult}_{L_{j}} \Sigma \text {. }
$$

ДоКАЗАТЕЛЬСтво получим непосредственным применением лемм 2.8-2.10.

СлЕДСТВИЕ 2.4. Инволюиия $\tau=\alpha_{L}$ или $\beta_{Y}$ удовлетворяет неравенству $n_{+}<n$ тогда и только тогда, когда $L$ или $Y$ есть максимальная кривая линейной системы $\Sigma$ соответственно, где $\Sigma^{+} \subset\left|n_{+} H\right|-$ собственный прообраз $\Sigma$ относительно $\tau$.

СлеДСТВИЕ 2.5. Предположим, что $n_{+}=n$. Тогда $\tau=\alpha_{L}$ для некоторой прямой $L \in \mathscr{L}$ и существуют такие прямые $L_{1}, L_{2} \in \mathscr{L}$, что $L \cup L_{1} \cup L_{2}=$ $P \cap V$, где $P \subset Q$ - некоторая плоскость.

Теперь докажем бирациональную жесткость многообразия $V$ и сюръективность $\varepsilon$. Предположим, что $c_{\mathrm{virt}}(\Sigma)<n$ для некоторой подвижной линейной системы $\Sigma$. Согласно предложению 2.17 , существует кривая $B \in \mathscr{B}$ такая, что $\operatorname{mult}_{B} \Sigma>n$. Пусть $\tau \in \varepsilon(G)$ - соответствующая инволюция (т. е. $\tau=\alpha_{L}$, если $B=L \in \mathscr{L}$, и $\tau=\beta_{Y}$, если $B=Y \in \mathscr{C}$ ). Согласно следствию $2.4, \Sigma^{+} \subset\left|n_{+} H\right|$, причем $n_{+}<n$, где $\Sigma^{+}-$собственный прообраз $\Sigma$ относительно $\tau$. Итерируя эту процедуру, построим последовательность инволюций $\tau_{i} \in \varepsilon(G)$ таких, что собственные прообразы $\Sigma^{(i)} \subset\left|n_{i} H\right|$ системы $\Sigma$ относительно композиций $\tau_{i} \ldots \tau_{1}$ удовлетворяют неравенствам $n_{i}<n_{i-1}$. Поскольку $n_{i} \in \mathbb{Z}_{+}$, на некотором шаге мы уже больше не сможем понизить порог $c\left(\Sigma^{(i)}, V\right)$. Следовательно, для некоторого $k \geqslant 1$ получим

$$
c\left(\Sigma^{(k)}, V\right)=c_{\mathrm{virt}}\left(\Sigma^{(k)}, V\right)=c_{\mathrm{virt}}(\Sigma, V),
$$

что и означает бирациональную жесткость. Более того, если зафиксировать бирациональный автоморфизм $\chi \in \operatorname{Bir} V$ и взять в качестве $\Sigma$ собственный прообраз системы $|H|$ относительно $\chi$, то описанная выше процедура дает равенство $n_{k}=1$ для некоторого $k$, т. е. $\Sigma^{(k)} \subset|H|$. Сравнивая размерности, получаем $\Sigma^{(k)}=|H|$, откуда вытекает, что $\tau_{k} \ldots \tau_{1} \chi \in \operatorname{Aut} V$ - бирегулярное отображение. Этим доказана сюръективность отображения $\varepsilon$.

Последний шаг в доказательстве теоремы 2.3 - установить, что $\varepsilon$ имеет тривиальное ядро.

Для удобства обозначений записываем слова в алфавите $A_{L}, B_{Y}$, используя заглавные буквы, а соответствующие бирациональные отображения - используя строчные буквы, например, $t=\varepsilon(T)$ и т. д. Для автоморфизма $t \in \operatorname{Bir} V$ определим целое число $n(t) \in \mathbb{Z}_{+}$формулой $\Sigma \subset|n(t) H|$, где $\Sigma$ - собственный прообраз системы $|H|$ относительно $t$; очевидно, что $n(t)=1$ тогда и только тогда, когда $t \in$ Aut $V$. Теорема 2.3 немедленно следует из следующего факта.

ПРЕДЛОЖЕНИЕ 2.18. Пусть $W=T_{1} \ldots T_{l}$ - произвольное слово в алфавите $\left\{A_{L}, B_{Y} \mid L \in \mathscr{L}, Y \in \mathscr{C}\right\}$. Если $w \in$ Aut $V$, то, используя соотношения в $R^{+}$, можно преобразовать слово $W$ в пустое слово. 
ДокАЗАТЕЛЬСтво. Обозначим через $W_{i}, i \leqslant l(W)=l$, левый отрезок слова $W$ длины $i$, т. е. $W_{i}=T_{1} \ldots T_{i}$. Положим

$$
\begin{aligned}
n^{*}(W) & =\max \left\{n\left(w_{i}\right) \mid 1 \leqslant i \leqslant l(W)\right\}, \\
\omega(W) & =\sharp\left\{i \mid n\left(w_{i}\right)=n^{*}(W), 1 \leqslant i \leqslant l(W)\right\} .
\end{aligned}
$$

Теперь поставим в соответствие каждому слову $W$ упорядоченную тройку

$$
\left(n^{*}(W), \omega(W), l(W)\right) .
$$

Введем на множестве слов отношение порядка, полагая $W>W^{\prime}$, если либо $n^{*}(W)>n^{*}\left(W^{\prime}\right)$, либо $n^{*}(W)=n^{*}\left(W^{\prime}\right)$ и $\omega(W)>\omega\left(W^{\prime}\right)$, либо $n^{*}(W)=n^{*}\left(W^{\prime}\right)$, $\omega(W)=\omega\left(W^{\prime}\right)$ и $l(W)>l\left(W^{\prime}\right)$. Легко видеть, что каждая убывающая цепь слов $W^{(1)}>W^{(2)}>\cdots$ обрывается. Следовательно, достаточно показать, что если $w \in$ Aut $V$, то слово $W$ можно преобразовать в такое слово $W^{\prime}$, что $W>W^{\prime}$, $w=w^{\prime}$.

Если слово $W$ содержит подслово $A_{L} A_{L}$ или $B_{Y} B_{Y}$, то, удаляя это подслово, получим меньшее слово $W^{\prime}$ (поскольку образ каждого левого отрезка слова $W^{\prime}$ совпадает с образом некоторого левого отрезка слова $W$ и отображение множества левых отрезков слова $W^{\prime}$ в множество левых отрезков $W$ инъективно).

Поэтому можно предполагать, что $W$ не содержит подслов $A_{L} A_{L}$ или $B_{Y} B_{Y}$.

Поскольку $n(w)=1$, можно предположить, что $n^{*}(W) \geqslant 2$ (иначе нечего доказывать). Пусть $s=\min \left\{i \mid n\left(w_{i}\right)=n^{*}(W)\right\} \leqslant l(W)-1$. Рассмотрим два случая $T_{s}=A_{L}$ и $T_{s}=B_{Y}$ по отдельности.

Случай 1: $T_{s}=B_{Y}$. В этом случае $n\left(w_{s-1}\right)=n\left(w_{s} \beta_{Y}\right)<n\left(w_{s}\right)$, согласно выбору $s$. В силу следствия $1.1, \operatorname{mult}_{Y} \Sigma_{s}>n\left(w_{s}\right)$, где $\Sigma_{s}-$ собственный прообраз $|H|$ относительно $w_{s}$. Поскольку по построению $n\left(w_{s+1}\right) \leqslant n\left(w_{s}\right)$, получаем $T_{s+1}=T_{s}=B_{Y}$. Это противоречит нашему предположению, что $W$ не содержит подслов $A_{L} A_{L}$ и $B_{Y} B_{Y}$.

Случай 2: $T_{s}=A_{L}$. В силу выбора $s$ получаем

$$
\operatorname{mult}_{L} \Sigma_{s}>n\left(w_{s}\right) .
$$

По предположению, $T_{s+1} \neq T_{s}$ и $n\left(w_{s+1}\right) \leqslant n\left(w_{s}\right)$. Согласно следствию 2.5 , $T_{s+1}=A_{L^{\prime}}$, где $L^{\prime} \subset V$ - такая прямая, что существует третья прямая $Z \subset V$ такая, что

$$
L \cup L^{\prime} \cup Z=P \cap V
$$

для некоторой плоскости $P \subset Q$.

Лемма 2.13. (i) $Z$ - максимальная прямая отображения $w_{s-1}$, т.е. въполнено неравенство $\operatorname{mult}_{Z} \Sigma_{s-1}>n\left(w_{s-1}\right)$. Следовательно,

$$
n\left(w_{s-1} \alpha_{Z}\right)<n\left(w_{s-1}\right) .
$$

(ii) Выполнено неравенство $n\left(w_{s-1} \alpha_{Z}\right)-\operatorname{mult}_{L^{\prime}} \Sigma^{\prime}=n\left(w_{s}\right)-\operatorname{mult}_{L^{\prime}} \Sigma_{s} \leqslant 0$, где $\Sigma^{\prime}$ - собственный прообраз $|H|$ относительно $w_{s-1} \alpha_{Z}$. Следовательно,

$$
n\left(w_{s-1} \alpha_{Z} \alpha_{L^{\prime}}\right) \leqslant n\left(w_{s-1} \alpha_{Z}\right) .
$$


ДокАзАТЕЛьство: прямые вычисления, основанные на лемме 2.12. Мы рассмотрим только утверждение (i), оставляя доказательство (ii) читателю. Поскольку $w_{s}=w_{s-1} \alpha_{L}$, получаем $w_{s-1}=w_{s} \alpha_{L}$ и по лемме 2.12

$$
\begin{gathered}
n\left(w_{s-1}\right)=n\left(w_{s} \alpha_{L}\right)=4 n\left(w_{s}\right)-3 \operatorname{mult}_{L} \Sigma_{s}, \\
\operatorname{mult}_{Z} \Sigma_{s-1}=2 n\left(w_{s}\right)-2 \operatorname{mult}_{L} \Sigma_{s}+\operatorname{mult}_{L^{\prime}} \Sigma_{s} .
\end{gathered}
$$

Следовательно, $n\left(w_{s-1}\right)-\operatorname{mult}_{Z} \Sigma_{s-1}=2 n\left(w_{s}\right)-\operatorname{mult}_{L} \Sigma_{s}-\operatorname{mult}_{L^{\prime}} \Sigma_{s}<0$, что нам и нужно. В случае (ii) рассуждения аналогичны. Лемма доказана.

Завершим, наконец, доказательство теоремы 2.3. Сначала рассмотрим случай, когда mult $L^{\prime} \Sigma_{s}>n\left(w_{s}\right)$. Используя соотношения $A_{Z}^{2}=e$ и $A_{Z} A_{L^{\prime}} A_{L}=$ $A_{L} A_{L^{\prime}} A_{Z}$, можно заменить подслово $A_{L} A_{L^{\prime}}$ подсловом $A_{Z} A_{L^{\prime}} A_{L} A_{Z}$. Эта операция увеличивает длину слова. Обозначим новое слово через $W^{+}$.

Очевидно, что $W_{i}^{+}=W_{i}$ при $i \leqslant s-1$. Далее,

$$
w_{s}^{+}=w_{s-1} \alpha_{Z}, \quad w_{s+1}^{+}=w_{s-1} \alpha_{Z} \alpha_{L^{\prime}}
$$

и $w_{s+2}^{+}=w_{s-1} \alpha_{Z} \alpha_{L^{\prime}} \alpha_{L}=w_{s+1} \alpha_{Z}$, так что $w_{s+i}^{+}=w_{s+i-2}$ при $i \geqslant 3$. Согласно лемме 2.13, $n\left(w_{i}^{+}\right)<n\left(w_{s}\right)=n^{*}(W)$ при $i=s, s+1, s+2$ (и по построению это верно также и для меньших значений $i<s)$. Следовательно, если $\omega(W) \geqslant 2$, то $n^{*}\left(W^{+}\right)=n^{*}(W)$ и $\omega\left(W^{+}\right)=\omega(W)-1$. Если $\omega(W)=1$, то $n^{*}\left(W^{+}\right)<n^{*}(W)$. В любом случае $W^{+}<W$.

Остается рассмотреть случай $\operatorname{mult}_{L^{\prime}} \Sigma_{s}=n\left(w_{s}\right)$. В этом случае $n\left(w_{s+1}\right)=$ $n\left(w_{s}\right), \operatorname{mult}_{L^{\prime}} \Sigma_{s+1}=n\left(w_{s+1}\right)$. Поскольку по предположению отсутствуют подслова $A_{L^{\prime}} A_{L^{\prime}}$, обязательно имеем $T_{s+2}=A_{Z}$. Теперь заменим подслово $T_{s} T_{s+1} T_{s+2}=A_{L} A_{L^{\prime}} A_{Z}$ подсловом $A_{Z} A_{L^{\prime}} A_{L}$. Обозначим новое слово через $W^{+}$. Оно имеет ту же длину, и по лемме 2.12 получаем неравенства $n\left(w_{i}^{+}\right)<n^{*}(W)$ при $i=s, s+1, s+2$. Рассуждая как в предыдущем случае, завершаем доказательство теоремы 2.3.

\section{Глава 3. Гиперкасательные дивизоры и линейные системы}

\section{§1. Определения и первый пример}

1.1. Гиперкасательные дивизоры. Техника гиперкасательных дивизоров и ее модификация - техника гиперкасательных линейных систем являются наиболее эффективным инструментом исключения бесконечно близких максимальных особенностей. Чтобы показать, как она работает, докажем бирациональную сверхжесткость общей гладкой гиперповерхности Фано $V=V_{M} \subset \mathbb{P}^{M}$ степени $M$ [64]. Как было показано в п. 2.1 главы 2, подвижная линейная система на $V$ не может иметь максимальных подмногообразий. Поэтому для доказательства бирациональной сверхжесткости достаточно исключить бесконечно близкий случай. В силу $4 n^{2}$-неравенства (§ 2 главы 2 ) бесконечно близкая максимальная особенность исключается следующим утверждением. 
ПРЕДЛОЖЕНИЕ 3.1. (i) Пусть о $\in V$ - точка, через которую проходит не более конечного числа прямых $L \subset V$. Тогда для любого неприводимого подмногообразия $Y \subset V$ коразмерности 2 выполнена оценка

$$
\frac{\operatorname{mult}_{o} Y}{\operatorname{deg} Y} \leqslant \frac{4}{M} .
$$

(ii) Cуществует открытое (в смысле топологии Зариского) множество $U \subset \mathbb{P}\left(H^{0}\left(\mathbb{P}^{M}, \mathscr{O}_{\mathbb{P} M}(M)\right)\right)$ гиперповерхностей степени $M$, удовлетворяющих условию конечности (i) в каждой точке.

Для упрощения обозначений в дальнейшем вместо

$$
\frac{\operatorname{mult}_{o} Y}{\operatorname{deg} Y}
$$

пишем

$$
\frac{\text { mult }_{o}}{\operatorname{deg}} Y
$$

(и аналогично в других случаях), объединяя кратность и степень в один символ.

ЗАМЕчАНИЕ 3.1. Предложение 3.1 немедленно исключает бесконечно близкую максимальную особенность подвижной системы $\Sigma$ на $V$ : пусть $B \subset V$ центр максимальной особенности, $o \in B$ - точка общего положения, $D_{1}, D_{2} \in \Sigma$ - общие дивизоры, $Z=\left(D_{1} \circ D_{2}\right)$ - алгебраический цикл их теоретико-схемного пересечения. Тогда $\operatorname{deg} Z=M n^{2}$, mult $_{o} Z>4 n^{2}$, причем $Z$ - эффективный цикл коразмерности 2 , что противоречит предложению 3.1 .

ДоКАЗАТЕЛЬСТВо ПРЕДЛОЖЕНИЯ 3.1. Пусть $\left(z_{1}, \ldots, z_{M}\right)$ - система аффинных координат на $\mathbb{P}=\mathbb{P}^{M}$ с началом в точке $o$. Запишем уравнение гиперповерхности $V$ :

$$
f=q_{1}+q_{2}+\cdots+q_{M}
$$

где $q_{i}$ однородны степени $i$ по $z_{*}$. Отметим, что прямые, проходящие через точку $x$ на гиперповерхности $V$, задаются системой уравнений

$$
q_{1}=q_{2}=\cdots=q_{M}=0 .
$$

Следовательно, множество общих нулей (26), самое большее, одномерно. Обозначим через

$$
f_{i}=q_{1}+\cdots+q_{i}
$$

левые отрезки разложения Тейлора уравнения $f$. Ясно, что на открытом аффинном подмножестве $\mathbb{A}=\mathbb{A}_{\left(z_{1}, \ldots, z_{M}\right)}^{M} \subset \mathbb{P}$ алгебраическое множество

$$
f_{1}=f_{2}=\cdots=f_{M}=0
$$

совпадает с (26), а потому имеет размерность не больше единицы. Отсюда следует, в свою очередь, что алгебраическое множество

$$
\left.f_{1}\right|_{\mathbb{A} \cap V}=\left.f_{2}\right|_{\mathbb{A} \cap V}=\cdots=\left.f_{M-1}\right|_{\mathbb{A} \cap V}=0
$$


на аффинной части гиперповерхности $V$ также не более чем одномерно: в теоретико-схемном смысле оно совпадает с (26), так что имеет носителем объединение прямых на $V$, проходящих через точку $о$.

Рассмотрим дивизоры

$$
D_{i}=\overline{\left\{\left.f_{i}\right|_{\mathbb{A} \cap V}=0\right\}},
$$

$i=1, \ldots, M-1$. Назовем их гиперкасательными дивизорами: пусть $H \in$ Pic $V$ - класс гиперплоского сечения, тогда, очевидно,

$$
D_{i} \in|i H|
$$

и

$$
\operatorname{mult}_{o} D_{i} \geqslant i+1,
$$

поскольку на аффинной части $V$

$$
\left.D_{i}\right|_{\mathbb{A} \cap V}=\left\{\left.\left(q_{i+1}+\cdots+q_{M}\right)\right|_{V}=0\right\} .
$$

Теперь по предположению

$$
\operatorname{dim}_{o}\left(D_{1} \cap \cdots \cap D_{M-1}\right) \leqslant 1,
$$

где $\operatorname{dim}_{o}$ обозначает размерность в окрестности точки $о$.

Построим последовательность различных индексов $i(1), i(2), \ldots, i(M-4)$ и последовательность неприводимых подмногообразий

$$
Y_{0}=Y, Y_{1}, \ldots, Y_{M-4}
$$

таких, что

$-Y_{k+1} \subset Y_{k}, \operatorname{dim} Y_{k}=M-3-k, \operatorname{codim}_{V} Y_{k}=k+2$;

- $Y_{k} \not \subset D_{i(k)}$, так что $\left(Y_{k} \circ D_{i(k)}\right)$ есть эффективный цикл на $V$ и $Y_{k+1}-$ одна из его неприводимых компонент;

- имеет место оценка

$$
\frac{\text { mult }_{o}}{\operatorname{deg}} Y_{k+1} \geqslant \frac{\text { mult }_{o}}{\operatorname{deg}} Y_{k} \cdot \frac{i(k)+1}{i(k)}
$$

для каждого $k=1, \ldots, M-4$. Построение осуществляется по индукции: при $k=1$ по соображениям размерности найдется гиперкасательный дивизор $D_{i(1)} \not \supset Y$. Точно так же выбирается дивизор $D_{i(k)}$ на каждом шаге.

Поскольку $Y_{k} \not \subset D_{i(k)}$, имеем

$$
\begin{gathered}
\operatorname{deg}\left(Y_{k} \circ D_{i(k)}\right)=i(k) \operatorname{deg} Y_{k}, \\
\operatorname{mult}_{o}\left(Y_{k} \circ D_{i(k)}\right) \geqslant(i(k)+1) \text { mult }_{o} Y_{k},
\end{gathered}
$$

так что найдется неприводимая компонента $Y_{k+1}$ цикла $\left(Y_{k} \circ D_{i(k)}\right)$, удовлетворяющая оценке (27).

Теперь кривая $C=Y_{M-4}$ удовлетворяет неравенству

$$
\frac{\text { mult }_{o}}{\operatorname{deg}} C \geqslant \frac{\text { mult }_{o}}{\operatorname{deg}} Y \cdot \prod_{k=1}^{M-4} \frac{i(k)+1}{i(k)} .
$$


Минимум выражения в правой части достигается при $i(k) \equiv k+3$ и равен $\left(\right.$ mult $\left._{o} / \operatorname{deg}\right) Y \cdot(M / 4)$. Левая часть не превосходит 1 , что и завершает доказательство части (i). Доказательство части (ii) отложим до п. 1.3.

Формализуя проведенное доказательство, рассмотрим следующую общую ситуацию: $X$ - неприводимое проективное многообразие, $o \in X-$ некоторая точка, причем о либо неособа, либо является изолированной особенностью. Пусть $\varphi: X^{+} \rightarrow X$ - раздутие точки $o, E=\varphi^{-1}(o)-$ исключительный дивизор. Предполагаем, что $E$ приведен и неприводим. Пусть $H-$ некоторый обильный дивизор на $X$, причем линейная система $|H|$ свободна, т. е. задает конечный морфизм

$$
\alpha=\alpha_{|H|}: X \rightarrow \mathbb{P}^{\operatorname{dim}|H|} .
$$

Степени подмногообразий $Y \subset X$ понимаются в смысле дивизора $H$, т. е. $\operatorname{deg} Y=\left(Y \cdot H^{\operatorname{dim} Y}\right)$, в частности, степень самого многообразия $X$ есть $\operatorname{deg} X=$ $H^{\operatorname{dim} X}=\operatorname{deg} \alpha \cdot \operatorname{deg} \alpha(X)$. Следующий факт хорошо известен.

Лемма 3.1. Для любого подмногообразия $Y \subset X$ справедливо неравенство mult $_{y} Y \leqslant \operatorname{deg} Y$, где $y \in Y-$ произвольная точка.

ОПРЕДЕЛЕНИЕ 3.1. Эффективный дивизор $D$ на $X$ называется гиперкасательным (относительно точки $о$ ), если $D^{+} \in|k H-l E|$, где $l \geqslant k+1, D^{+}-$ собственный прообраз дивизора $D$ на $X^{+}$. Число $\beta(D)=l / k>1$ назовем наклоном дивизора $D$.

ПримеР 3.1. Пусть $X \subset \mathbb{P}^{N}$ - гиперповерхность степени $d \geqslant 2, f=q_{1}+$ $q_{2}+\cdots+q_{d}-$ ее уравнение относительно системы аффинных координат $\left(z_{1}, \ldots, z_{N}\right)$ с началом в точке $o, q_{i}$ однородны степени $i$. Пусть $D_{i}-$ замыкание множества

$$
\left.\left(q_{1}+\cdots+q_{i}\right)\right|_{X}=0,
$$

$i \leqslant d-1$, и предположим, что $\left.q_{i+1}\right|_{E} \not \equiv 0$ (где $\left(z_{1}: \ldots: z_{N}\right)$ рассматриваются как однородные координаты на исключительном дивизоре раздутия точки $o$ в $\left.\mathbb{P}^{N}\right)$. Тогда $D_{i}$ - гиперкасательный дивизор с наклоном $(i+1) / i$. Если же $q_{i+1} \equiv \cdots \equiv q_{k-1} \equiv 0$, но $\left.q_{k}\right|_{E} \not \equiv 0$, то наклон равен $k / i$.

Пусть $\mathscr{D}$ - конечное множество гиперкасательных дивизоров, $\sharp \mathscr{D}=N \leqslant$ $\operatorname{dim} X-1$. Предположим, что имеет место равенство

$$
\operatorname{codim}_{o}\left(\bigcap_{D \in \mathscr{D}} D\right)=\sharp \mathscr{D},
$$

где $\operatorname{codim}_{o}$ обозначает коразмерность в произвольно малой окрестности точки $o$.

ПРЕДЛОЖЕНИЕ 3.2. Имеет место оценка

$$
\frac{\text { mult }_{o}}{\operatorname{deg}} Y \leqslant\left(\min _{\mathscr{B} \subset \mathscr{D}} * \prod_{D \in \mathscr{B}} \beta(D)\right)^{-1},
$$

где символ тіп* означает, что минимум берется по всем подмножествам $\mathscr{B} \subset \mathscr{D}$ мощности

$$
\sharp \mathscr{B}=\min \{\operatorname{dim} Y-1, N-\operatorname{codim} Y\}=b .
$$


ДокАЗАТЕЛЬСтво повторяет приведенное выше рассуждение для гиперповерхности Фано $V_{M} \subset \mathbb{P}^{M}:$ строим последовательность неприводимых многообразий $Y_{0}=Y, Y_{1}, \ldots, Y_{b}$ и гиперкасательных дивизоров $D_{1}, \ldots, D_{b}$, где $Y_{i} \not \subset D_{i+1}$ и $Y_{i+1}$ - неприводимая компонента эффективного цикла $\left(Y_{i} \circ D_{i+1}\right)$, удовлетворяющая неравенству

$$
\frac{\text { mult }_{o}}{\operatorname{deg}} Y_{i+1} \geqslant \beta\left(D_{i+1}\right) \frac{\text { mult }_{o}}{\operatorname{deg}} Y_{i} .
$$

Доказательство завершается применением леммы $3.1 \mathrm{k}$ подмногообразию $Y_{b}$.

1.2. Гиперкасательные линейные системы. В некоторых случаях бывает удобнее работать не с гиперкасательными дивизорами, а с линейными системами, порожденными этими дивизорами. По аналогии с определением 3.1 дадим

ОПредЕЛЕНИЕ 3.2. Непустая линейная система $\Sigma$ на $X$ называется гиперкасательной (относительно точки $о$ ), если $\Sigma^{+} \subset|k H-l E|$, где $l \geqslant k+1, \Sigma^{+}-$ собственный прообраз системы $\Sigma$ на $X^{+}$. Число $\beta(\Sigma)=l / k>1$ назовем наклоном системы $\Sigma$.

Важнейшая характеристика гиперкасательной линейной системы $\Sigma$ - коразмерность ее базисного множества в окрестности точки $o, \operatorname{codim}_{o} \operatorname{Bs} \Sigma$.

Множество гиперкасательных дивизоров $D \in \mathscr{D}, D^{+} \in\left|k_{D} H-l_{D} E\right|$, порождает гиперкасательную систему $\Sigma_{k}=\Sigma_{k}(\mathscr{D})$ следующим образом. Пусть

$$
f_{D} \in H^{0}\left(X, \mathscr{O}_{X}\left(k_{D} H\right)\right)
$$

- сечение, задающее дивизор $D$. Положим

$$
\Sigma_{k}=\left|\sum_{k_{D} \leqslant k} f_{D} s_{D}=0\right|,
$$

где суммирование ведется по всем гиперкасательным дивизорам $D \in \mathscr{D}$ таким, что $k_{D} \leqslant k, s_{D} \in \alpha^{*} H^{0}\left(\mathbb{P}^{\operatorname{dim}|H|}, \mathscr{O}\left(k-k_{D}\right)\right)$ - подъем произвольного многочлена степени $\left(k-k_{D}\right)$, имеющего нуль порядка $\left(k-k_{D}\right)$ в точке $\alpha(o)$. Легко видеть, что

$$
\beta\left(\Sigma_{k}\right) \geqslant \min _{D \in \mathscr{D}, k_{D} \leqslant k}\left\{\frac{k+l_{D}-k_{D}}{k}\right\} .
$$

Кроме того, поскольку $\alpha$ - конечный морфизм, имеем равенство

$$
\operatorname{codim}_{o} \operatorname{Bs} \Sigma_{k}=\sharp\left\{D \in \mathscr{D} \mid k_{D} \leqslant k\right\} .
$$

Отсюда, в частности, видно, что целочисленная функция $\operatorname{codim}_{o} \operatorname{Bs} \Sigma_{k}$ возрастает при $k=k_{D}$ для некоторого $D \in \mathscr{D}$ и только для таких значений $k$. Определим упорядочивающую функиию

$$
\chi:\{1, \ldots, N\} \rightarrow \mathscr{K}=\left\{k_{D} \mid D \in \mathscr{D}\right\}
$$

соотношением

$$
\sharp\left\{D \in \mathscr{D} \mid k_{D}<\chi(i)\right\}<i \leqslant \sharp\left\{D \in \mathscr{D} \mid k_{D} \leqslant \chi(i)\right\} .
$$

Например, $\chi(1)=\min \left\{k_{D} \mid D \in \mathscr{D}\right\}, \chi(N)=\max \left\{k_{D} \mid D \in \mathscr{D}\right\}$. 
Лемма 3.2. Имеет место равенство

$$
\operatorname{codim}_{o} \operatorname{Bs} \Sigma_{\chi(i)} \geqslant i \text {. }
$$

ДокАзАтЕльство. Утверждение леммы есть прямое следствие формулы $(29)$ и явной конструкции гиперкасательной системы $\Sigma_{j}$.

Удобство работы с упорядочивающей функцией состоит в том, что для общего набора гиперкасательных дивизоров

$$
\mathbb{D}=\left(D_{1}, \ldots, D_{N}\right) \in \prod_{i=1}^{N} \Sigma_{\chi(i)}
$$

и произвольного подмногообразия $Y$ коразмерности $l$, содержащего точку $o$, имеем $Y \not \subset \operatorname{supp} D_{i}$ при $i \geqslant l+1$. В частности, корректно определен эффективный цикл $\left(Y \circ D_{l+1}\right)$ коразмерности $l+1$, удовлетворяющий неравенству

$$
\operatorname{mult}_{o}\left(Y \circ D_{l+1}\right) \geqslant \beta\left(\Sigma_{l+1}\right) \frac{\operatorname{mult}_{o}}{\operatorname{deg}} Y \operatorname{deg}\left(Y \circ D_{l+1}\right) .
$$

Ввиду линейности этого неравенства по циклу $\left(Y \circ D_{l+1}\right)$ существует неприводимая компонента этого цикла - неприводимое подмногообразие $Y_{1}$ коразмерности $l+1$, удовлетворяющее неравенству

$$
\frac{\text { mult }_{o}}{\operatorname{deg}} Y_{1} \geqslant \beta\left(\Sigma_{l+1}\right) \frac{\text { mult }_{o}}{\operatorname{deg}} Y .
$$

Повторяя эту процедуру $b=\min \{\operatorname{dim} Y-1, N-\operatorname{codim} Y\}$ раз, получаем неприводимое подмногообразие $Y_{b}$, удовлетворяющее неравенству

$$
\frac{\text { mult }_{o}}{\operatorname{deg}} Y_{b} \geqslant \frac{\text { mult }_{o}}{\operatorname{deg}} Y \prod_{i=1}^{b} \beta\left(\Sigma_{l+i}\right) .
$$

Применяя к подмногообразию $Y_{b}$ лемму 3.1 , получаем следующее предложение.

ПРЕДЛОЖЕНИЕ 3.3. Имеет место оценка

$$
\frac{\text { mult }_{o}}{\operatorname{deg}} Y \leqslant\left(\prod_{i=1}^{b} \beta\left(\Sigma_{l+i}\right)\right)^{-1} .
$$

Техника гиперкасательных дивизоров и линейных систем применяется в большинстве случаев к подмногообразиям малой коразмерности - в основном к дивизорам и подмногообразиям коразмерности два, и в процессе образования последовательных пересечений участвуют почти все дивизоры (соответственно линейные системы). Более того, почти во всех конкретных ситуациях для каждого $D \in \mathscr{D}$ имеем $l_{D}=k_{D}+1$, так что все наклоны имеют вид

$$
\beta(D)=\frac{k_{D}+1}{k_{D}}
$$

и аналогично для гиперкасательных систем. Легко видеть, что для таких значений наклонов предложения 3.2 и 3.3 дают одинаковые оценки. Однако в наиболее трудных случаях требуется рассуждать тоньше, например, важно, чтобы первый пересекающий гиперкасательный дивизор был взят из системы $\Sigma_{l}$, 
а не $\Sigma_{l+1},-$ в некоторых случаях это можно обосновать, привлекая дополнительные соображения, см. [66], [67], [77], [86]. Поэтому в более трудных случаях техника гиперкасательных линейных систем эффективнее и гибче техники гиперкасательных дивизоров.

1.3. Условия регулярности. Применение техники гиперкасательных дивизоров/линейных систем основано на предположении (28). Поэтому эта техника работает успешно только в том случае, когда можно доказать, что общее многообразие заданного семейства удовлетворяет условию (28) в каждой своей точке. Для завершения доказательства бирациональной сверхжесткости общей гиперповерхности Фано $V=V_{M} \subset \mathbb{P}=\mathbb{P}^{M}$ проверим, что (28) действительно справедливо в каждой точке $o \in V$ при условии общности гиперповерхности $V$.

На самом деле выполнено несколько более сильное условие. Чтобы его сформулировать, рассмотрим систему аффинных координат $\left(z_{1}, \ldots, z_{M}\right)$ с центром в точке $o \in V$. Пусть $f=q_{1}+q_{2}+\cdots+q_{M}-$ уравнение гиперповерхности $V$, где $q_{i}\left(z_{*}\right)$ - однородный многочлен степени $i$.

ОПРЕДЕЛЕНИЕ 3.3. Точка $o \in V$ регулярна, если многочлены $q_{1}, \ldots, q_{M-1}$ образуют регулярную последовательность в $\mathscr{O}_{o, \mathbb{P}}$, т. е. система уравнений

$$
q_{1}=q_{2}=\cdots=q_{M-1}=0
$$

задает конечное множество прямых в $\mathbb{P}$, проходящих через $o \in \mathbb{P}$.

Очевидно, что свойство регулярности не зависит от выбора системы координат. Очевидно, далее, что если точка $o \in V$ регулярна, то на $V$ имеется не более конечного числа прямых, проходящих через точку о. Поэтому часть (ii) предложения 3.1, а вместе с ней и бирациональная сверхжесткость общей гиперповерхности Фано вытекают из следующего факта.

ПРЕДЛОЖЕНИЕ 3.4. Общая гиперповерхность $V=V_{M} \subset \mathbb{P}$ регулярна в каждой точке $о \in V$.

ЗАмЕчАНИЕ 3.2. Общность понимается в смысле топологии Зариского на $\mathbb{P}\left(H^{0}\left(\mathbb{P}, \mathscr{O}_{\mathbb{P}}(M)\right)\right)$.

ДокАЗАТЕЛЬство. Пусть $\mathscr{P}_{k, K}$ - пространство однородных многочленов степени $k$ от $K$ переменных, $\operatorname{dim} \mathscr{P}_{k, K}=\left(\begin{array}{c}k+K-1 \\ K-1\end{array}\right)$. Рассмотрим пространство пар

$$
S=\left\{(x, F) \mid x \in \mathbb{P}, F \in H^{0}\left(\mathbb{P}, \mathscr{O}_{\mathbb{P}}(M)\right)\right\}
$$

с естественными проекциями $p: S \rightarrow \mathbb{P}$ и $q: S \rightarrow H^{0}\left(\mathbb{P}, \mathscr{O}_{\mathbb{P}}(M)\right)$. Выделим в нем гиперповерхность инцидентности $I=\{(x, F) \mid F(x)=0\}$. Пусть $Y \subset I$ состоит из таких пар $(x, F)$, что точка $x$ не является $(M-1)$-регулярной на гиперповерхности $F=0$. Предложение 3.4 будет доказано, если мы установим, что $q(Y)$ - замкнутое собственное подмножество пространства однородных многочленов $\mathscr{P}_{M, M+1}$. Последнее утверждение, в свою очередь, вытекает из следующего факта.

Лемма 3.3. Коразмерность множества $Y(x)=Y \cap p^{-1}(x)$ в слое $p^{-1}(x)=$ $H^{0}\left(\mathbb{P}, \mathscr{O}_{\mathbb{P}}(M)\right)$ равна $M+1$. 
ДоКАЗАТЕЛЬСтво ЛЕммы. Зафиксируем произвольную точку $x \in \mathbb{P}$ и систему аффинных координат $\left(z_{1}, \ldots, z_{M}\right)$ с началом в $x$. Пусть

$$
Y_{k}(x)=\left\{\left(q_{1}, \ldots, q_{k}\right) \in \prod_{i=1}^{k} \mathscr{P}_{i, M} \mid \operatorname{codim}\left\{q_{1}=\cdots=q_{k}=0\right\}<k\right\} .
$$

Очевидно, $Y(x)=Y_{M-1}(x)$, так что достаточно установить, что $\operatorname{codim} Y_{k}(x)=$ $M+1$ для $k=1, \ldots, M-1$ (имеется в виду коразмерность относительно слоя $\left.p^{-1}(x)\right)$. Сделаем это индукцией по $k$.

Для $k=1$ утверждение очевидно (гладкие гиперповерхности существуют!).

В предположении, что codim $Y_{k}(x)=M+1$ для некоторого $k \in\{1, \ldots, M-2\}$, докажем, что то же самое справедливо и для $k+1$. Положим

$$
R_{k}=\prod_{i=1}^{k} \mathscr{P}_{i, M} \backslash Y_{k}
$$

Пусть

$$
\pi: \prod_{i=1}^{k+1} \mathscr{P}_{i, M}=\prod_{i=1}^{k} \mathscr{P}_{i, M} \times \mathscr{P}_{k+1, M} \rightarrow \prod_{i=1}^{k} \mathscr{P}_{i, M}
$$

- естественная проекция. Поскольку коразмерность пересечения $I \cap p^{-1}(x)$ относительно слоя $p^{-1}(x)$ есть 1 , нам достаточно показать, что

$$
\operatorname{codim} Y_{k+1} \cap \pi^{-1}\left(R_{k}\right) \geqslant M
$$

(коразмерность берется относительно пространства многочленов $\prod_{i=1}^{k+1} \mathscr{P}_{i, M}$ ). В свою очередь, это следует из неравенства

$$
\mu_{k+1}=\operatorname{codim}_{\pi^{-1}\left(q_{*}\right)}\left(\pi^{-1}\left(q_{*}\right) \cap Y_{k+1}\right) \geqslant M,
$$

где $q_{*}=\left(q_{1}, \ldots, q_{k}\right) \in R_{k}-$ произвольная регулярная последовательность. Это последнее неравенство мы и будем доказывать.

Естественно, $\pi^{-1}\left(q_{*}\right) \cong \mathscr{P}_{k+1, M}$. Условие

$$
q_{k+1} \in \pi^{-1}\left(q_{*}\right) \cap Y_{k+1}
$$

означает, что $q_{k+1}$ обращается в нуль на одной из компонент замкнутого алгебраического множества $\left\{q_{1}=\cdots=q_{k}=0\right\} \subset \mathbb{A}^{M}$. Поскольку последовательность $q_{*}$ регулярна, все компоненты этого множества - конусы коразмерности $k$ с вершинами в точке $x=(0, \ldots, 0)$. Проективизируя, получаем замкнутое проективное множество $Q=\bigcup Q_{i} \subset \mathbb{P}^{M-1}$, все компоненты которого имеют коразмерность $k$.

Пусть $S \subset \mathbb{P}^{M-1}-(k-1)$-плоскость общего положения, так что ограничение соответствующей линейной проекции $\pi_{S}: \mathbb{P}^{M-1} \rightarrow \mathbb{P}^{M-1-k}$ на каждую компоненту $Q_{i}$ множества $Q$ есть конечный морфизм. Очевидно, что ограничение пространства однородных многочленов

$$
\pi_{S}^{*} H^{0}\left(\mathbb{P}^{M-1-k}, \mathscr{O}_{\mathbb{P} M-1-k}(k+1)\right)
$$


на каждую компоненту $Q_{i}$ инъективно. Таким образом, мы явно построили пространство однородных многочленов $W_{k+1} \subset \mathscr{P}_{k+1, M}$, пересекающееся с $Y_{k+1}$ только по нулю. Следовательно,

$$
\mu_{k+1} \geqslant \operatorname{dim} W_{k+1}=\left(\begin{array}{c}
M \\
k+1
\end{array}\right) \geqslant M .
$$

Лемма 3.3, а вместе с ней и предложение 3.4 доказаны.

\section{§ 2. Полные пересечения Фано}

2.1. Теорема о бирациональной сверхжесткости. Рассмотрим полное пересечение Фано

$$
\mathbb{P}=\mathbb{P}^{M+k} \supset V=V_{d_{1} \cdots d_{k}}=F_{1} \cap F_{2} \cap \cdots \cap F_{k},
$$

где $F_{i} \subset \mathbb{P}$ - гиперповерхности степени $d_{i} \geqslant 2$, причем целые числа $d_{i}, d_{1} \leqslant$ $d_{2} \leqslant \cdots \leqslant d_{k}$, удовлетворяют соотношению $d_{1}+\cdots+d_{k}=M+k, M \geqslant 4, k \geqslant 2$. Многообразие $V$ предполагается гладким $M$-мерным, так что $\operatorname{Pic} V=\mathbb{Z} H$ по теореме Лефшеца, где $H$ - класс гиперплоского сечения, $K_{V}=-H$, так что $V-$ многообразие Фано индекса 1.

ТЕОрема 3.1. При $M \geqslant 2 k+1$ общее (в смысле топологии Зариского) многообразие $V$ является бирачионально сверхжестким.

В данном пункте будет точно сформулировано условие общности положения для $V$, в следующем - построены гиперкасательные системы и доказана бирациональная сверхжесткость.

Пусть $\left(z_{1}, \ldots, z_{M+k}\right)$ - система аффинных координат на $\mathbb{P}$ с началом в точке $o \in V$. Запишем

$$
\begin{aligned}
& f_{1}\left(z_{*}\right)=q_{1,1}+q_{1,2}+\cdots+q_{1, d_{1}}, \\
& \ldots \ldots \ldots \cdots \cdots+\cdots \cdots \cdots+\cdots \cdots+\cdots \\
& f_{i}\left(z_{*}\right)=q_{i, 1}+q_{i, 2}+\cdots+q_{i, d_{i}},
\end{aligned}
$$

- уравнения гиперповерхностей $F_{1}, \ldots, F_{k}$, разложенные на однородные по $z_{*}$ компоненты $q_{i, j}$.

ОПРЕДЕлЕНИЕ 3.4. Точка $o \in V$ регулярна, если набор многочленов $\left\{q_{i, j} \mid\right.$ $\left.1 \leqslant i \leqslant k, 1 \leqslant j \leqslant d_{i},(i, j) \neq\left(k, d_{k}\right)\right\}$, состоящий из всех многочленов $q_{i, j}$, кроме $q_{k, d_{k}}$, образует регулярную последовательность в $\mathscr{O}_{o, \mathbb{P}}$, т. е. система уравнений $\left\{q_{i, j}=0 \mid(i, j) \neq\left(k, d_{k}\right)\right\}$ задает конечное число прямых в $\mathbb{P}$, проходящих через точку $о$.

В частности, на $V$ лежит, самое большее, конечное число прямых, проходящих через регулярную точку $o \in V$.

Пусть

$$
\mathscr{H}=\prod_{i=1}^{k}\left(H^{0}\left(\mathbb{P}, \mathscr{O}_{\mathbb{P}}\left(d_{i}\right)\right) \backslash\{0\}\right)
$$

- множество упорядоченных наборов $k$ ненулевых однородных многочленов $\left(f_{1}, \ldots, f_{k}\right)$ степеней $d_{1}, \ldots, d_{k}$ соответственно. 
ПРЕДЛОЖЕНИЕ 3.5. Существует непустое открытое по Зарискому множество $U_{\text {reg }} \subset \mathscr{H}$ такое, что для $\left(f_{1}, \ldots, f_{k}\right) \in U_{\text {reg }}$ полное пересечение $V=V\left(f_{1}, \ldots, f_{k}\right) \subset \mathbb{P}$ регулярно в каждой точке.

Доказательство дано ниже, в $\S 3$.

ДоКАЗАТЕЛЬСТВо ТЕОРЕМЫ 3.1. В силу предложения 3.5 достаточно показать, что полное пересечение $V$, регулярное в каждой точке, бирационально сверхжесткое. Зафиксируем такое многообразие $V$.

В силу пп. 2.2 главы 2, бирациональная сверхжесткость следует из неравенства

$$
\frac{\text { mult }_{o}}{\operatorname{deg}} Y \leqslant \frac{4}{\operatorname{deg} V}=\frac{4}{d_{1} \cdots d_{k}},
$$

которое должно быть выполнено для любого неприводимого подмногообразия $Y \subset V$ и любой точки $o \in Y$. Зафиксировав пару $o \in Y$, будем доказывать (30). Отметим, что оценка (30) точна: подмногообразие $H_{1} \cap H_{2} \cap V$, где $H_{i}$ - различные касательные гиперплоскости к $V$ в точке $o$, имеет степень $\operatorname{deg} V$ и кратность 4 в точке $о$.

Пусть $\Lambda_{1}^{\mathbb{P}}=\overline{\left\{\lambda_{1} q_{1,1}+\cdots+\lambda_{k} q_{k, 1}=0\right\}}$ - линейная система касательных гиперплоскостей к $V$ в точке $o$ (черта сверху означает проективное замыкание в $\mathbb{P})$. Пусть $\Lambda_{1}=\left.\Lambda_{1}^{\mathbb{P}}\right|_{V}$ - ее ограничение на $V$.

Лемма 3.4. Имеет место равенство $\operatorname{codim}_{V} \operatorname{Bs} \Lambda_{1}=\operatorname{codim}_{\mathbb{P}} \operatorname{Bs} \Lambda_{1}^{\mathbb{P}}=k$.

ДоказАтельство. Легко видеть, что

$$
\left.q_{i, 1}\right|_{V}=-\left.\left(q_{1,2}+\cdots+q_{i, d_{i}}\right)\right|_{V} .
$$

Положим $\mathbb{T}=T_{o} V=\left\{q_{1,1}=q_{2,1}=\cdots=q_{k, 1}=0\right\} \cong \mathbb{C}^{M}$. Квадратичные компоненты функций $\left.q_{i, 1}\right|_{V}$ суть $\left.\left(-q_{i, 2}\right)\right|_{\mathbb{T}}$ (относительно любой системы локальных параметров в точке $о$ ). Поскольку последовательность

$$
q_{1,1}, \ldots, q_{k, 1}, q_{1,2}, \ldots, q_{k, 2}
$$

регулярна, заключаем, что $\operatorname{Bs} \Lambda_{1}=\mathbb{T} \cap V$ есть замкнутое множество коразмерности точно $k$ в окрестности точки о. Положим $T_{i}=\left\{q_{i, 1}=0\right\}$, так что $\mathbb{T}=T_{1} \cap \cdots \cap T_{k}$.

Мы утверждаем, что для каждого $i, 1 \leqslant i \leqslant k$, замкнутое алгебраическое множество $R_{i}=T_{1} \cap \cdots \cap T_{i} \cap V$ есть неприводимое подмногообразие коразмерности $i$ в $V$. Это верно при $i=1$ по теореме Лефшеца, поскольку $V$ гладкое и группа $\operatorname{Pic} V$ порождена классом гиперплоского сечения, так что $T_{1} \cap V$ должно быть неприводимо. Предположим, что наше утверждение справедливо для каждого $j, 1 \leqslant j \leqslant i$, где $i \leqslant k-1$. Имеем $R_{i} \not \subset T_{i+1}$, поскольку это верно в окрестности точки $x \in R_{i}$ и $R_{i}$ неприводимо. Следовательно, теоретико-схемное пересечение $R_{i}$ и $T_{i+1}$ есть эффективный цикл $\left(R_{i} \circ T_{i+1}\right)$ коразмерности $i+1$. Однако по теореме Лефшеца группа циклов коразмерности $i+1<M / 2$ по модулю численной эквивалентности порождена классом $\left(R_{i} \circ T_{i+1}\right)$. Следовательно, $\left(R_{i} \circ T_{i+1}\right)=R_{i} \cap T_{i+1}=R_{i+1}$ есть неприводимый цикл, что нам и нужно.

В итоге получаем, что Bs $\Lambda_{1}$ есть неприводимое подмногообразие коразмерности $k$. Лемма 3.4 доказана. 
СлЕДСТвиЕ 3.1. Предположим, что неприводимое подмногообразие $Y \subset V$ коразмерности 2 не удовлетворяет неравенству (30). Тогда существует неприводимое подмногообразие $R \subset V$ коразмерности $k+1$, удовлетворяющее неравенству

$$
\frac{\text { mult }_{o}}{\operatorname{deg}} R \geqslant 2^{k-1} \frac{\text { mult }_{o}}{\operatorname{deg}} Y .
$$

ДокАЗАТЕЛЬСтво. Рассуждая по образцу п. 1.1, строим последовательность неприводимых подмногообразий $Y_{0}=Y, Y_{1}, \ldots, Y_{k-2}, \operatorname{codim} Y_{i}=i+2$, удовлетворяющих неравенствам

$$
\frac{\text { mult }_{o}}{\operatorname{deg}} Y_{i} \geqslant 2^{i} \frac{\text { mult }_{o}}{\operatorname{deg}} Y,
$$

$Y_{i+1}$ - неприводимая компонента цикла $\left(Y_{i} \circ T_{j(i)}\right)$, где $j(i) \in\{1, \ldots, k\}$. Такое построение возможно в силу предыдущей леммы. Теперь имеется альтернатива: либо $Y_{k-2}=\operatorname{Bs} \Lambda_{1}$, либо $Y_{k-2} \neq \operatorname{Bs} \Lambda_{1}$. В первом случае в силу условия регулярности $Y_{k-2}$ - неприводимое подмногообразие степени $\operatorname{deg} V$, имеющее в точке $o$ кратность в точности $2^{k}$ (касательный конус $T_{o} Y_{k-2}$ есть пересечение квадрик $\left.\left.q_{1,2}\right|_{\mathbb{T}}=\cdots=\left.q_{k, 2}\right|_{\mathbb{T}}=0\right)$, так что $\left(\right.$ mult $\left._{o} / \operatorname{deg}\right) Y \leqslant 4 / \operatorname{deg} V$ вопреки предположению. Значит, реализуется второй случай, т. е. найдется дивизор $T_{j(k-2)} \not \supset Y_{k-2}$. В качестве $R$ возьмем неприводимую компоненту эффективного цикла $\left(Y_{k-2} \circ T_{j(k-2)}\right)$, удовлетворяющую неравенству

$$
\frac{\text { mult }_{o}}{\operatorname{deg}} R \geqslant 2 \frac{\text { mult }_{o}}{\operatorname{deg}} Y_{k-2} .
$$

Следствие доказано.

2.2. Построение гиперкасательных дивизоров. Зафиксируем неприводимое многообразие $R$ коразмерности $k+1$, удовлетворяющее неравенству (31).

Пусть $\mathbb{A} \subset \mathbb{P}$ - аффинная карта с координатами $\left(z_{1}, \ldots, z_{M+k}\right), o=(0, \ldots, 0)$. Положим

$$
f_{i, \alpha}=q_{i, 1}+\cdots+q_{i, \alpha} .
$$

Символ $s_{a}, a \in \mathbb{Z}_{+}$, обозначает произвольный однородный многочлен степени $a$ от $\left(z_{*}\right)$. Рассмотрим линейные системы

$$
\Lambda_{i, j}^{\mathbb{A}}=\left|\sum_{\alpha=1}^{\min \left\{j, d_{i}-1\right\}} f_{i, \alpha} s_{j-\alpha}\right|
$$

на $\mathbb{A}$. Беря замыкание общего дивизора системы $\Lambda_{i, j}^{\mathbb{A}}$ в $\mathbb{P}$, получаем линейную систему $\Lambda_{i, j}^{\mathbb{P}}$ на $\mathbb{P}$. Очевидно, что

$$
\operatorname{Bs} \Lambda_{i, j}^{\mathbb{A}}=\left\{q_{i, \alpha}=0 \mid 1 \leqslant \alpha \leqslant \min \left\{j, d_{i}-1\right\}\right\}, \quad \operatorname{Bs} \Lambda_{i, j}^{\mathbb{P}}=\overline{\operatorname{Bs} \Lambda_{i, j}^{\mathbb{A}}} .
$$

Положим $\Lambda_{j}^{\mathbb{P}}=\sum_{i=1}^{k} \Lambda_{i, j}^{\mathbb{P}}$. Для этой линейной системы имеем

$$
\left.\operatorname{Bs} \Lambda_{j}^{\mathbb{P}}=\overline{\left\{q_{i, \alpha}=0 \mid 1 \leqslant \alpha \leqslant \min \left\{j, d_{i}-1\right\}\right.}\right\}
$$


И

$$
c(j)=\operatorname{codim}_{\mathbb{P}} \operatorname{Bs} \Lambda_{j}^{\mathbb{P}}=\sum_{i=1}^{k} \min \left\{j, d_{i}-1\right\} .
$$

Имеем $\operatorname{Bs} \Lambda_{d_{k}-1}^{\mathbb{P}}=\operatorname{Bs} \Lambda_{d_{k}}^{\mathbb{P}}=\cdots=\operatorname{Bs}_{\infty}^{\mathbb{P}}$ и $\operatorname{codim}_{\mathbb{P}} \operatorname{Bs}_{\infty}^{\mathbb{P}}=M$.

ОПРЕДЕЛЕНИЕ 3.5. Линейная система

$$
\Lambda_{j}=\left.\Lambda_{j}^{\mathbb{P}}\right|_{V}
$$

называется $j$-й гиперкасателъной системой в точке $о$.

Например, $\Lambda_{1}$ есть $(k-1)$-мерная линейная система на $V$, высекаемая на $V$ касательными гиперплоскостями в точке $o$.

Теперь получим оценку для коразмерности базисного множества гиперкасательных систем. Априори можно лишь утверждать, что $\operatorname{codim}_{V} \operatorname{Bs}_{j} \geqslant$ $\operatorname{codim}_{\mathbb{P}} \operatorname{Bs} \Lambda_{j}^{\mathbb{P}}-k$ : когда система ограничивается с $\mathbb{P}$ на $V$, коразмерность базисного множества может уменьшиться. Однако в нашем случае условие регулярности улучшает положение.

Система $\Lambda_{1}$ была построена и использована выше.

Лемма 3.5. (i) При $j \in\left\{2, \ldots, d_{k}-2\right\}$

$$
\operatorname{codim}_{V} \operatorname{Bs} \Lambda_{j}=\operatorname{codim}_{\mathbb{P}} \operatorname{Bs} \Lambda_{j}^{\mathbb{P}}=c(j)
$$

в окрестности точки о.

(ii) Для каждого ј имеет место оченка

$$
\operatorname{codim}_{V} \operatorname{Bs} \Lambda_{j} \geqslant \operatorname{codim}_{\mathbb{P}} \operatorname{Bs} \Lambda_{j}^{\mathbb{P}}-1 .
$$

ДокАзАТЕЛьство. Легко видеть, что при $\alpha \leqslant d_{i}-1$

$$
\left.f_{i, \alpha}\right|_{V \cap \mathbb{A}}=-\left.\left(q_{i, \alpha+1}+\cdots+q_{i, d_{i}}\right)\right|_{V \cap \mathbb{A}} .
$$

Напомним, что $\mathbb{T}=T_{o} V \cong \mathbb{C}^{M}$ - касательное пространство. Введем вспомогательные линейные системы

$$
\Lambda_{i, j}^{\mathbb{T}}=\left|\sum_{\alpha=1}^{\min \left\{j, d_{i}-1\right\}} q_{i, \alpha+1} s_{j-\alpha}\right|_{\mathbb{T}}, \quad \Lambda_{j}^{\mathbb{T}}=\sum_{i=1}^{k} \Lambda_{i, j}^{\mathbb{T}} .
$$

Это линейные системы конусов степени $j+1$ на касательном пространстве $\mathbb{T}$.

Заменяя общий дивизор линейной системы его касательным конусом в заданной точке, получаем касательную линейную систему. Эта простая операция всегда имеет смысл. Однако на уровне явных уравнений имеется потенциальный источник неприятностей: заменяя уравнение его первой однородной компонентой, необходимо проследить, что последняя не есть тождественный нуль.

Мы утверждаем, что

$$
\Lambda_{j}^{\mathbb{T}}=T_{o} \Lambda_{j}
$$


В самом деле, в силу (34) уравнение касательного конуса к дивизору $\left(\left.f_{i, \alpha}\right|_{V \cap \mathbb{A}}\right)$ в точке о есть $\left.q_{i, \alpha+1}\right|_{\mathbb{T}}$, если последнее отлично от нуля. Поэтому в диаграмме равенств

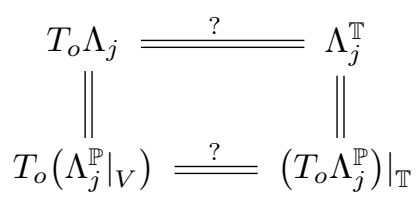

мы вправе стереть вопросительные знаки и получить желаемое равенство (35) при условии, что система $\Lambda_{j}^{\mathbb{T}}$ ненулевая. Но последнее верно в силу условия регулярности. (Условие $\Lambda_{j}^{\mathbb{T}} \neq 0$ существенно слабее, чем условие регулярности; ниже условие регулярности используется в полную силу.) Очевидно, что

$$
\operatorname{Bs} \Lambda_{j}^{\mathbb{T}}=\left\{\left.q_{i, \alpha+1}\right|_{\mathbb{T}}=0,1 \leqslant \alpha \leqslant \min \left\{j, d_{i}-1\right\}\right\} \subset \mathbb{T} .
$$

Снова в силу условия регулярности получаем

$$
\operatorname{codim}_{\mathbb{T}} \operatorname{Bs} \Lambda_{j}^{\mathbb{T}}=\sum_{i=1}^{k} \min \left\{j, d_{i}-1\right\}=\operatorname{codim}_{\mathbb{P}} \operatorname{Bs} \Lambda_{j}^{\mathbb{P}}
$$

при $j \in\left\{1, \ldots, d_{k}-2\right\}$. Случай $j=d_{k}-1$ невозможен, так как многочлен $q_{k, d_{k}}$ исключен из условия регулярности. Поскольку, очевидно, для любой линейной системы $\Sigma$ имеем

$$
T_{o} \operatorname{Bs} \Sigma \subset \operatorname{Bs} T_{o} \Sigma
$$

получаем утверждение (i).

Докажем (ii). Базисное множество системы $\Lambda_{j}^{\mathbb{P}}$ задается системой $c(j)$ уравнений и имеет коразмерность $c(j)$, см. (32). Базисное множество системы $\Lambda_{j}$ задается теми же самыми уравнениями, ограниченными на $V$, и $c(j) \leqslant \operatorname{dim} V$. Отсюда следует, что $\operatorname{codim}_{V} \operatorname{Bs} \Lambda_{j}-\operatorname{codim}_{\mathbb{P}} \operatorname{Bs} \Lambda_{j}^{\mathbb{P}}$ есть невозрастающая функция параметра $j$. (При $a \geqslant b$ коразмерность $\operatorname{codim}_{V} \operatorname{Bs} \Lambda_{a}$ превышает $\operatorname{codim}_{V} \operatorname{Bs} \Lambda_{b}$ на, самое большее, число новых уравнений, т. е. $c(a)-c(b)$.) Поэтому достаточно доказать (ii) для $j=\infty:\left.\operatorname{dim}\left(\operatorname{Bs}_{\infty}^{\mathbb{P}}\right)\right|_{V} \leqslant 1$.

В самом деле, базисная подсхема $\left.\left(\mathrm{Bs}_{\infty}^{\mathbb{P}}\right)\right|_{V}$ задается на $V$ системой уравнений

$$
\left\{q_{i, \alpha}=0 \mid 1 \leqslant \alpha \leqslant d_{i}-1\right\} .
$$

Следовательно, как подсхема проективного пространства $\mathbb{P}$ подсхема $\left.\left(\mathrm{Bs}_{\infty}^{\mathbb{P}}\right)\right|_{V}$ задается системой $M+k$ уравнений

$$
\left\{q_{i, \alpha}=0 \mid 1 \leqslant \alpha \leqslant d_{i}\right\} .
$$

В силу условия регулярности, $\left.\operatorname{dim}\left(\mathrm{Bs}_{\infty}^{\mathbb{P}}\right)\right|_{V} \leqslant 1$, что нам и нужно. Лемма 3.5 доказана.

Для $j \geqslant 1$ положим $w^{+}(j)=\sharp\left\{i, 1 \leqslant i \leqslant k \mid j \leqslant d_{i}-1\right\}$. Далее, положим $w(j)=w^{+}(j)-1$, если $j=1,2$ и $d_{k}-1$, и $w(j)=w^{+}(j)$ для остальных значений $j$. Поскольку $d_{i} \geqslant 2$ для каждого $i=1, \ldots, k$, получаем $w^{+}(1)=k$. Далее,

$$
c(j)=\sharp\left\{(i, \alpha) \mid 1 \leqslant i \leqslant k, 1 \leqslant \alpha \leqslant \min \left\{j, d_{i}-1\right\}\right\}=\sum_{\alpha=1}^{j} w^{+}(\alpha)
$$


для каждого $j \geqslant 1$. Для $1 \leqslant j \leqslant d_{k}-1$ рассмотрим пространства

$$
\Delta_{j}=\underbrace{\Lambda_{j} \times \cdots \times \Lambda_{j}}_{w(j) \text { раз }},
$$

параметризующие упорядоченные наборы $\left(D_{j, 1}, \ldots, D_{j, w(j)}\right) \in \Delta_{j}$, состоящие из $w(j)$ дивизоров линейных систем $\Lambda_{j}$. Положим

$$
\Delta=\prod_{j=2}^{d_{k}-1} \Delta_{j}
$$

Лемма 3.6. Для общего набора $\left(D_{*, *}\right) \in \Delta$ теоретико-множественное пересечение

$$
R^{*}=R \cap\left(\bigcap_{j=2}^{d_{k}-1} \bigcap_{a=1}^{w(j)} D_{j, a}\right)
$$

одномерно в окрестности точки о.

ДокАЗАТЕЛьство. Прежде всего, отметим, что мы рассматриваем пересечение

$$
w(2)+\cdots+w\left(d_{k}-1\right)=c\left(d_{k}-1\right)-k-2=M-k-2
$$

дивизоров с $R$, так что $\operatorname{dim} R^{*} \geqslant 1$. Для $b \in\left\{2, \ldots, d_{k}-1\right\}$ положим

$$
R_{b}=R \cap\left(\bigcap_{j=2}^{b} \bigcap_{a=1}^{w(j)} D_{j, a}\right) .
$$

Докажем (возрастающей) индукцией по $b$, что для общего набора $\left(D_{*, *}\right) \in$ $\prod_{j=2}^{b} \Delta_{j}$ имеем

$$
\operatorname{codim}_{o} R_{b}=2+\sum_{j=2}^{b} w(j),
$$

где $\operatorname{codim}_{o}$ обозначает коразмерность в окрестности точки $о$. Положим для $e \in\{0,1, \ldots, w(b)\}$

$$
R_{b, e}=R \cap\left(\bigcap_{j=2}^{b-1} \bigcap_{a=1}^{w(j)} D_{j, a}\right) \cap\left(\bigcap_{a=1}^{e} D_{b, a}\right) .
$$

$\mathrm{B}$ частности, $R_{b, w(b)}=R_{b+1,0}=R_{b}$. Докажем индукцией по $e$, что для общего набора $\left(D_{*, *}\right)$ выполнено равенство

$$
\operatorname{codim}_{o} R_{b, e}=k+1+\sum_{j=2}^{b-1} w(j)+e .
$$

При $b=2, e=0$ равенство (37) выполнено по предположению, поскольку $R_{2,0}=R$. Возьмем $e \leqslant w(b)-1$ и предположим, что (37) выполнено. В силу (33)

$$
\operatorname{codim}_{V} \operatorname{Bs} \Lambda_{b}=c(b)=\sum_{j=1}^{b} w^{+}(j)=k+1+\sum_{j=2}^{b-1} w(j)+w(b),
$$


так что общий дивизор $D_{b, e+1} \in \Lambda_{b}$ не содержит компонент множества $R_{b, e}$, содержащих точку $о$. Это доказывает (37).

Из равенства (37) сразу следует (36). Лемма 3.6 доказана.

Применим теперь построенную последовательность гиперкасательных дивизоров к оценке кратности mult $_{o} R$.

ЛЕмма 3.7. В каждом из замкнутых алгебраических множеств $R_{b, e}$ имеется такая неприводимая компонента $Y_{b, e}$, что последовательность неприводимых подмногообразий $\left\{Y_{b, e} \mid 2 \leqslant b \leqslant d_{k}-1,0 \leqslant e \leqslant w(b)\right\}$ имеет следующие свойства:

(i) $Y_{2,0}=R$;

(ii) $Y_{b, e} \ni$ о и, в частности,

$$
\operatorname{codim}_{V} Y_{b, e}=k+1+\sum_{j=2}^{b-1} w(j)+e
$$

(iii) $n p u e \leqslant w(b)-1$

$$
\frac{\operatorname{mult}_{o}}{\operatorname{deg}} Y_{b, e+1} \geqslant \frac{b+1}{b} \cdot \frac{\text { mult }_{o}}{\operatorname{deg}} Y_{b, e} .
$$

ДокАЗАТЕЛЬСтво повторяет рассуждения п. 1.2.

СлеДСтвиЕ 3.2. Справедлива оченка

$$
\frac{\text { mult }_{o}}{\operatorname{deg}} R \leqslant \frac{3 d_{k}}{2 d_{k}-2} \cdot 2^{w^{+}(2)} \cdot\left(\prod_{d_{i} \geqslant 3} d_{i}\right)^{-1} .
$$

ДокАзАТЕльство. Рассуждаем, как в п. 1.2.

Последнее подмногообразие $Y_{b, e}$ в построенной последовательности есть неприводимая кривая. Ее кратность в точке $о$ не превосходит ее степени. Применяя часть (iii) леммы 3.7 , получаем оценку

$$
\frac{\operatorname{mult}_{o}}{\operatorname{deg}} R \cdot \prod_{b=2}^{d_{k}-1}\left(\frac{b+1}{b}\right)^{w(b)} \leqslant 1 .
$$

Вспоминая определение чисел $w(b)$ и $w^{+}(b)$, получим:

$$
\begin{aligned}
\prod_{b=2}^{d_{k}-1}\left(\frac{b+1}{b}\right)^{w(b)} & =\frac{2}{3} \frac{d_{k}-1}{d_{k}} \prod_{b=2}^{d_{k}-1}\left(\frac{b+1}{b}\right)^{w^{+}(b)} \\
& =\frac{2 d_{k}-2}{3 d_{k}} \cdot 2^{-w^{+}(2)} \cdot\left(\prod_{b=3}^{d_{k}} b^{w^{+}(b-1)-w^{+}(b)}\right) .
\end{aligned}
$$

Однако $w^{+}(b-1)-w^{+}(b)=\sharp\left\{i \mid 1 \leqslant i \leqslant k, d_{i}=b\right\}$. Следовательно, произведение в скобках равно

$$
\prod_{d_{i} \geqslant 3} d_{i}
$$

что и требуется. Следствие доказано. 
Вспоминая, что подмногообразие $R$ удовлетворяет неравенству (31), получаем оценку

$$
\frac{\text { mult }_{o}}{\operatorname{deg}} C \leqslant \frac{3 d_{k}}{4 d_{k}-4} \frac{4}{\operatorname{deg} V} .
$$

Из неравенства $M \geqslant 2 k+1$ легко следует, что $d_{k} \geqslant 4$. Этим неравенство (30) и бирациональная сверхжесткость многообразия $V$ полностью доказаны. Теорема 3.1 доказана.

2.3. Циклические накрытия. Опишем для полноты картины метод построения гиперкасательных дивизоров для многообразия Фано $V$, реализованного в виде циклического накрытия степени $K \geqslant 2$

$$
\sigma: V \stackrel{K: 1}{\longrightarrow} X \subset \mathbb{P} .
$$

Здесь $\mathbb{P}=\mathbb{P}^{M}$ - проективное пространство, $X$ - гладкое многообразие Фано, $\sigma$ разветвлено над гладким дивизором $W \cap X$, где $W=W_{K l} \subset \mathbb{P}$ - гиперповерхность степени $K l$, заданная уравнением $g\left(x_{*}\right)=0,\left(x_{*}\right)=\left(x_{0}: \ldots: x_{M}\right)-$ однородные координаты на $\mathbb{P}, g$ - многочлен степени $K l$. Вводя новую координату $u$ веса $l$, можно реализовать $V$ как подмногообразие во взвешенном проективном пространстве

$$
V \subset \mathbb{P}(\underbrace{1, \ldots, 1}_{M+1 \text { раз }}, l),
$$

добавляя к уравнениям многообразия $X$ уравнение

$$
u^{K}=g\left(x_{0}, \ldots, x_{M}\right) .
$$

Гиперкасательные дивизоры в точке $o \in V$ строятся по-разному в зависимости от того, лежит точка $p=\sigma(o) \in X$ на дивизоре ветвления или нет.

Предположим, что $p \notin W$. Выберем некоторую систему аффинных координат $\left(z_{1}, \ldots, z_{M}\right)$ с началом в этой точке. Без ограничения общности считаем, что $z_{i}=x_{i} / x_{0}$. Положим $y=u / x_{0}^{l}$. Теперь стандартное аффинное множество $\mathbb{A}_{\left(z_{1}, \ldots, z_{M}, y\right)}^{M+1}$ является картой для $\mathbb{P}(1, \ldots, 1, l)$. Несколько злоупотребляя обозначениями, используем для неоднородного многочлена, соответствующего $g$, тот же самый символ

$$
g=w_{0}+w_{1}+\cdots+w_{K l},
$$

где $w_{i}\left(z_{*}\right)$ - однородный многочлен степени $i$ и без ограничения общности полагаем $w_{0}=1$. Можно считать, что $y(o)=1\left(o \in V\right.$, так что $\left.y(o)^{K}=1\right)$. Положим

$$
\begin{aligned}
g^{1 / K}=\left(1+w_{1}+\cdots+w_{K l}\right)^{1 / K} & =1+\sum_{i=1}^{\infty} \gamma_{i}\left(w_{1}+\cdots+w_{K l}\right)^{i} \\
& =1+\sum_{i=1}^{\infty} \Phi_{i}\left(w_{1}, \ldots, w_{K l}\right)
\end{aligned}
$$


где $\gamma_{i} \in \mathbb{Q}$ определяются рядом Тейлора функции $(1+s)^{1 / K}$ в нуле, $s-$ переменная:

$$
(1+s)^{1 / K}=1+\sum_{i=1}^{\infty} \gamma_{i} s^{i}
$$

и $\Phi_{i}\left(w_{1}\left(z_{*}\right), \ldots, w_{K l}\left(z_{*}\right)\right)$ суть однородные многочлены степени $i \geqslant 1$ от переменных $z_{*}$. Легко видеть, что при $i \in\{1, \ldots, K l\}$ имеем

$$
\Phi_{i}\left(w_{*}\left(z_{*}\right)\right)=\frac{1}{K} w_{i}+\Phi_{i}^{\sharp}\left(w_{1}, \ldots, w_{i-1}\right) .
$$

Теперь множество гиперкасательных дивизоров строится таким образом: к имеющимся гиперкасательным дивизорам на многообразии $X$ в точке $p$ (точнее, их $\sigma$-прообразам) добавляются новые дивизоры, заданные уравнениями

$$
L_{j}=\overline{\left\{\left.\left(y-1-\sum_{i=1}^{j} \Phi_{i}\left(w_{1}, \ldots, w_{j}\right)\right)\right|_{V}=0\right\}}
$$

$j=l, l+1, \ldots, K l-1$. Черта сверху означает замыкание в $\mathbb{P}(1, \ldots, 1, l)$. Очевидно, что $L_{j}$ высекается на $V$ гиперповерхностью степени $j$ и имеет в точке $o$ кратность $\geqslant j+1$. (При $j<l$ ввиду присутствия координаты $y$ уравнение $L_{j}$ во взвешенном проективном пространстве имеет степень $l$, поэтому дивизоры $L_{j}$ не являются гиперкасательными.) Для использования новых гиперкасательных дивизоров требуются соответствующие условия регулярности: пересечение полного набора дивизоров должно иметь правильную размерность. Описанная выше конструкция ("извлечение корня") позволила доказать бирациональную жесткость многих классов многообразий Фано [65], [70], [73].

Если точка $p \in W$ лежит на дивизоре ветвления накрытия $\sigma$, то к гиперкасательным дивизорам многообразия $X$ в точке $p$ добавляются первые $K-1$ гиперкасательных дивизоров гиперповерхности $W$, т. е. дивизоры

$$
\sigma^{*}\left(\left.\left(w_{1}+\cdots+w_{j}\right)\right|_{X}=0\right)
$$

$j=1, \ldots, K-1$. Детали см. в [65], [70], [73].

\section{§ 3. Регулярные многообразия Фано}

В п. 1.3 было доказано, что общая гиперповерхность Фано удовлетворяет условиям регулярности. Однако приведенный в п. 1.3 метод доказательства не работает для полных пересечений Фано. Ниже приводится другой метод, позволяющий обосновать условия регулярности для полных пересечений.

3.1. Метод оценки коразмерности. Пусть $z_{1}, \ldots, z_{N+1}$ - некоторое множество переменных. Символ $\mathscr{P}_{a}$ обозначает пространство однородных многочленов степени $a$ от переменных $z_{*}$. Пусть

$$
\mathscr{L}=\prod_{i=1}^{l+1} \mathscr{P}_{m_{i}}=\left\{\left(p_{1}, \ldots, p_{l+1}\right)\right\}
$$


- множество всех наборов, состоящих из $(l+1)$ многочлена от переменных $z_{*}$, $0 \leqslant l \leqslant N-1$. Каждому набору из $(l+1)$ многочлена $\left(p_{*}\right) \in \mathscr{L}$ поставим в соответствие проективизированное множество его нулей

$$
Z\left(p_{*}\right)=\left\{p_{1}=\cdots=p_{l+1}=0\right\} \subset \mathbb{P}^{N}=\mathbb{P}\left(\mathbb{C}^{N+1}\right)=X .
$$

Мы пишем $Z\left(p_{*}\right)$, а не $V\left(p_{*}\right)$, чтобы избежать путаницы и отличить этот объект от полного пересечения $V=V\left(f_{*}\right)$. Пусть

$$
Y=\left\{\left(p_{*}\right) \in \mathscr{L} \mid \operatorname{codim}_{X} Z\left(p_{*}\right) \leqslant l\right\}
$$

- множество "нерегулярных" наборов длины $(l+1)$. Нужно оценить коразмерность множества $Y$. Случай $l=N-1$, когда “правильная" размерность множества $Z\left(p_{*}\right)$ равна нулю, особенно важен для приложений к многообразиям Фано. Однако по техническим соображениям удобнее рассмотреть общий случай произвольного $l \in\{0, \ldots, N-1\}$. Положим $I=\{1, \ldots, l+1\}$ и

$$
\mu_{j}=\min _{S \subset I, \sharp S=j}\left\{\sum_{i \in S} m_{i}\right\},
$$

$j=1, \ldots, l+1$. Предположим, что $m=\mu_{1}=\min \left\{m_{1}, \ldots, m_{l+1}\right\} \geqslant 2$.

ПРеДЛОЖЕНИЕ 3.6. Для любого $l \in\{1, \ldots, N-1\}$ справедлива следующая оценка:

$$
\operatorname{codim}_{\mathscr{L}} Y \geqslant \min _{j \in\{0, \ldots, l\}}\left\{\left(\mu_{j+1}-j\right)(N-j)+1\right\} .
$$

ЗАмЕчАНИЕ 3.3. При $l=0$ получаем тривиальную оценку $\operatorname{codim}_{\mathscr{L}} Y=$ $\operatorname{dim} \mathscr{P}_{m}$.

ДОКАЗАТЕЛЬСТВО ПРЕДЛОЖЕНИЯ 3.6. ПоЛОЖим

$$
\mathscr{L}_{a}=\prod_{i=1}^{a} \mathscr{P}_{m_{i}}=\left\{\left(p_{1}, \ldots, p_{a}\right)\right\} .
$$

Для каждого нерегулярного набора $\left(p_{1}, \ldots, p_{l+1}\right)$ длины $(l+1)$ зафиксируем первый (считая слево направо) момент, когда коразмерность множества нулей $p_{1}=\cdots=p_{a}=0$ принимает неправильное значение. Рассмотрим множества

$$
Y_{a}=\left\{\left(p_{*}\right) \in \mathscr{L}_{a} \mid \operatorname{codim}_{X} Z\left(p_{1}, \ldots, p_{a}\right)=\operatorname{codim}_{X} Z\left(p_{1}, \ldots, p_{a-1}\right)=a-1\right\} .
$$

Очевидным образом

$$
Y=\coprod_{a=1}^{l+1}\left(Y_{a} \times \prod_{i=a+1}^{l+1} \mathscr{P}_{m_{i}}\right) .
$$

В частности, $\operatorname{codim}_{\mathscr{L}} Y=\min \left\{\operatorname{codim}_{\mathscr{L}_{a}} Y_{a} \mid 1 \leqslant a \leqslant l+1\right\}$. Положим $I_{a}=$ $\{1, \ldots, a\} \subset I$ и

$$
\mu_{a, j}=\min _{S \subset I_{a}, \sharp S=j}\left\{\sum_{i \in S} m_{i}\right\},
$$


$j=1, \ldots, a$. Очевидно, что $\mu_{a, j} \geqslant \mu_{j}$. Поэтому достаточно доказать оценку

$$
\operatorname{codim}_{\mathscr{L}_{a}} Y_{a} \geqslant \min _{j \in\{0, \ldots, a-1\}}\left\{\left(\mu_{a, j+1}-j\right)(N-j)+1\right\}
$$

для каждого $a=2, \ldots, l+1$. Тривиальный случай $a=1$ опускаем, потому что в этом случае $\operatorname{codim} \mathscr{L}_{1} Y_{1}=\operatorname{dim} \mathscr{P}_{m_{1}} \geqslant \operatorname{dim} \mathscr{P}_{m}$, что заведомо больше, чем правая часть неравенства (39).

Пространство $\mathscr{L}_{a}$, множество $Y_{a}$ и неравенство (40) не зависят от $l$. Следовательно, можем упростить обозначения, полагая $a=l+1$ и $\mu_{a, j}=\mu_{j}$. Другими словами, докажем неравенство (39) для $Y_{l+1}$ вместо $Y$.

Обозначим $Y_{l+1}$ символом $Y^{*}$. Мы свели исходную проблему к более простой задаче оценки коразмерности множества $Y^{*}$ в $\mathscr{L}$, где $Y^{*}$ состоит изо всех таких наборов многочленов $\left(p_{1}, \ldots, p_{l+1}\right)$ длины $(l+1)$, что множество $p_{1}=\cdots=$ $p_{l}=0$ имеет правильную размерность и существует неприводимая компонента $B \subset Z\left(p_{1}, \ldots, p_{l}\right)$, на которой многочлен $p_{l+1}$ обращается в нуль. Пусть $\langle B\rangle-$ линейная оболочка множества $B$. Положим $b=\operatorname{codim}\langle B\rangle \leqslant l$.

Определим $Y^{*}(b)$ как множество всех таких наборов $\left(p_{*}\right) \in Y^{*}$ длины $(l+1)$, для которых существует компонента $B \subset Z\left(p_{1}, \ldots, p_{l}\right)$ такая, что

$$
\operatorname{codim}_{X}\langle B\rangle=b,\left.\quad p_{l+1}\right|_{B} \equiv 0 .
$$

Очевидно,

$$
Y^{*}=\bigcup_{b=0}^{l} Y^{*}(b) .
$$

Поэтому достаточно показать, что

$$
\operatorname{codim}_{\mathscr{L}} Y^{*}(b) \geqslant\left(\mu_{b+1}-b\right)(N-b)+1
$$

для каждого $b=0,1, \ldots, l$. Установим справедливость неравенства (41).

Случай $b=0$. Здесь $\langle B\rangle=\mathbb{P}^{N}$ и поэтому каждый ненулевой моном степени $m_{l+1} \geqslant m$ от линейных форм от переменных $z_{1}, \ldots, z_{N+1}$ не обращается в нуль на $B$. Пространство таких мономов

$$
\left\{\prod_{i=1}^{m_{l+1}}\left(a_{i, 1} z_{1}+\cdots+a_{i, N+1} z_{N+1}\right)\right\} \subset \mathscr{P}_{m_{l+1}}
$$

замкнуто. Его размерность есть $m_{l+1} N+1 \geqslant \mu_{1} N+1$. С другой стороны, множество многочленов $p_{l+1} \in \mathscr{P}_{m_{l+1}}$, обращающихся в нуль на $B$, замкнуто. Эти два замкнутых множества пересекаются только по нулю. Следовательно, коразмерность множества $Y(0)$ в $\mathscr{L}$ не меньше, чем $\mu_{1} N+1$. Это дает оценку (41) для $b=0$.

Случай $b \geqslant 1$. Здесь $\langle B\rangle=\mathbb{P}^{N-b}$. Наша стратегия состоит в том, чтобы свести этот случай к предыдущему $(b=0)$, ограничивая многочлены $p_{i}$ на линейную оболочку $P=\langle B\rangle$. Хотя наши рассуждения довольно простые, они не совсем очевидны и требуют некоторой дополнительной работы. 
ОПРЕДЕЛЕНИЕ 3.6. Пусть $g_{1}, \ldots, g_{e}$ - однородные многочлены на проективном пространстве $P, e \leqslant \operatorname{dim} P-1, \operatorname{deg} g_{i} \geqslant 2$ при $i=1, \ldots, e$. Назовем неприводимое подмногообразие $C \subset P$ такое, что $\langle C\rangle=P$ и $\operatorname{codim}_{P} C=e, a c$ соииированным подмногообразием последовательности $\left(g_{*}\right)$, если существует цепочка неприводимых подмногообразий $R_{j} \subset P, j=0,1, \ldots, e$, удовлетворяющая следующим требованиям:

- $R_{0}=P$

- для каждого $j=0,1, \ldots, e-1$ подмногообразие $R_{j+1}$ есть неприводимая компонента замкнутого алгебраического множества

$$
\left\{g_{j+1}=0\right\} \cap R_{j},
$$

где $\left.g_{j+1}\right|_{R_{j}} \not \equiv 0$, так что $\operatorname{codim}_{P} R_{j}=j$ для всех $j$;

$-R_{e}=C$.

Если последовательность $\left(g_{*}\right)$ имеет ассоциированное подмногообразие, то назовем ее хорошей.

Лемма 3.8. (i) Свойство быть хорошей является открытым.

(ii) Хорошая последовательность $\left(g_{*}\right)$ может иметь самое большее

$$
\left[\frac{1}{e+1} \prod_{j=1}^{e} \operatorname{deg} g_{j}\right]
$$

ассочиированных подмногообразий.

ДокАЗАТЕЛЬСтво легко получается индукцией по $e$. Для $g_{1}$ имеем условие $g_{1} \not \equiv 0$, которое очевидным образом открытое. Далее, по крайней мере одна неприводимая компонента гиперповерхности $g_{1}=0$ должна иметь степень не меньше двух, и это условие также является открытым. Таких компонент может быть самое большее $\left[(1 / 2) \operatorname{deg} g_{1}\right]$.

Предположим, что лемма справедлива для каждого $e=1, \ldots, j$, где $j \leqslant$ $\operatorname{dim} P-2$. Обозначим через $G_{j}$ открытое множество хороших последовательностей длины $j$. Согласно (ii), для каждой последовательности $\left(g_{*}\right) \in G_{j}$ имеется самое большее

$$
\left[\frac{1}{j+1} \prod_{\alpha=1}^{j} \operatorname{deg} g_{\alpha}\right]
$$

ассоциированных подмногообразий. Многочлен $g_{j+1}$ не должен обращаться в нуль по крайней мере на одном из них, скажем, $R_{j}$, и, более того, пересечение

$$
\left\{g_{j+1}=0\right\} \cap R_{j}
$$

должно содержать неприводимую компоненту, линейная оболочка которой есть $P$. Очевидно, это условие определяет открытое подмножество в

$$
G_{j} \times H^{0}\left(P, \mathscr{O}_{P}\left(\operatorname{deg} g_{j+1}\right)\right) .
$$

Каждое ассоциированное подмногообразие имеет коразмерность $j+1$ и не содержится в гиперплоскости; следовательно, его степень не меньше, чем $j+2$. Лемма 3.8 доказана. 
Вернемся теперь к многочленам $p_{*}$ и предположим, что $l>b$. Мы утверждаем, что среди них можно найти $(l-b)$ многочленов - после перенумерации можно считать, что это $p_{1}, \ldots, p_{l-b},-$ таких, что последовательность

$$
\left.p_{1}\right|_{P}, \ldots,\left.p_{l-b}\right|_{P}
$$

является хорошей и подмногообразие $B$ есть одно из ее ассоциированных подмногообразий.

В самом деле, предположим, что мы уже нашли $j$ многочленов - пусть это $p_{1}, \ldots, p_{j}$ - таких, что последовательность $\left(\left.p_{1}\right|_{P}, \ldots,\left.p_{j}\right|_{P}\right)$ является хорошей и одно из ее ассоциированных подмногообразий, скажем $R_{j}$, содержит $B$. Если $j<l-b$, то $R_{j} \neq B$ и существует многочлен $p_{\alpha}, \alpha \in\{j+1, \ldots, l\}$, такой, что

$$
\left.p_{\alpha}\right|_{R_{j}} \not \equiv 0
$$

В противном случае $R_{j} \subset Z\left(p_{1}, \ldots, p_{l}\right)$ и мы приходим к противоречию, поскольку $\operatorname{dim} R_{j}>\operatorname{dim} B$. После перенумерации можем считать, что $\alpha=j+1$. Теперь $\left.p_{j+1}\right|_{B} \equiv 0$, так что существует такая неприводимая компонента $R_{j+1}$ множества $\left\{p_{j+1}=0\right\} \cap R_{j}$, что $R_{j+1} \supset B$. Продолжая таким образом, получим наше утверждение.

Теперь зафиксируем проективное подпространство $P \subset \mathbb{P}^{N}$ коразмерности $b$. Пусть $Y^{*}(P)$ - множество всех таких наборов $\left(p_{1}, \ldots, p_{l+1}\right) \in Y^{*}$ длины $(l+1)$, что существует компонента $B \subset Z\left(p_{1}, \ldots, p_{l}\right)$, линейная оболочка которой есть $\langle B\rangle=P$ и $\left.p_{l+1}\right|_{B} \equiv 0$.

Согласно лемме 3.8 , хорошие последовательности образуют открытое множество. Поэтому можно оценивать коразмерность множества $Y^{*}(P)$ в $\mathscr{L}$, предполагая, что $\left(\left.p_{1}\right|_{P}, \ldots,\left.p_{l-b}\right|_{P}\right)$ образуют хорошую последовательность. Пусть

$$
B_{1}, \ldots, B_{K}
$$

- все ее ассоциированные подмногообразия, линейная оболочка которых есть $P$. Если $\left(p_{1}, \ldots, p_{l+1}\right) \in Y^{*}(P)$, то многочлены

$$
\left.p_{l-b+1}\right|_{P}, \ldots,\left.p_{l+1}\right|_{P}
$$

должны все обращаться в нуль на одном из этих подмногообразий $B_{i}$. Рассуждая теперь как в случае $b=0$, получаем

$$
N \sum_{j=l-b+1}^{l+1} \operatorname{deg} p_{j}+b+1 \geqslant \mu_{b+1}(N-b)+b+1
$$

независимых условий на $p_{l-b+1}, \ldots, p_{l+1}$. Принимая во внимание, что размерность грассманиана есть $\operatorname{dim} G(N+1-b, N+1)=b(N+1-b)$, получаем окончательно:

$$
\begin{aligned}
\operatorname{codim}_{\mathscr{L}} Y^{*}(b) & \geqslant \mu_{b+1}(N-b)+b+1-b(N+1-b) \\
& =\left(\mu_{b+1}-b\right)(N-b)+1
\end{aligned}
$$

что и требуется. 
В процессе доказательства мы предполагали, что $l>b$. Если $l=b$, то $B \subset \mathbb{P}^{N}$ - прямая, $l=N-1$ и неравенство (41) получается простым подсчетом размерностей: для фиксированной прямой $B$ условие $\left.p\right|_{B} \equiv 0$ на многочлен $p$ степени $e \geqslant 1$ определяет замкнутое алгебраическое множество многочленов коразмерности $e+1$ в $\mathscr{P}_{e}$. Следовательно,

$$
\operatorname{codim}_{\mathscr{L}} Y^{*}(N-1) \geqslant \sum_{i=1}^{N}\left(m_{i}+1\right)-2(N-1)=\mu_{l+1}-l+1,
$$

поскольку $\mu_{l+1}=m_{1}+\cdots+m_{N}$.

Предложение 3.6 доказано.

СлЕДСТвИЕ 3.3. В обозначениях предложения 3.6 для $l \leqslant N-2$ справедлива следующая оценка:

$$
\operatorname{codim}_{\mathscr{L}} Y \geqslant m N+1,
$$

в то время как для $l=N-1$ справедлива следующая оченка:

$$
\operatorname{codim}_{\mathscr{L}} Y \geqslant \min \left\{m N+1, \mu_{l+1}-l+1\right\} .
$$

ДокАЗАТЕЛЬСтво. Очевидно, что $\mu_{j} \geqslant j m$ для каждого $j=1, \ldots, l+1$. Поэтому

$$
\left(\mu_{j+1}-j\right)(N-j)+1 \geqslant \varepsilon(j)+m N+1
$$

где многочлен $\varepsilon(t)=-(m-1) t^{2}+(N m-N-m) t$ имеет два корня, $t=0$ и $t=N-1-1 /(m-1)$. Значит, $\varepsilon(0)=0$ и $\varepsilon(j) \geqslant 0$ при $j=1, \ldots, N-2$. Следовательно, можно опустить в (39) значения $j=1, \ldots, l-1$. Если $l \leqslant N-2$, можно опустить и значение $j=l$. Следствие доказано.

3.2. Доказательство предложения 3.5. Напомним, что $\mathscr{H}$ обозначает множество упорядоченных наборов $k$ ненулевых однородных многочленов $\left(f_{1}, \ldots, f_{k}\right)$ на $\mathbb{P}$ степеней $d_{1}, \ldots, d_{k}$ соответственно.

Прежде всего, существует открытое по Зарискому множество $U_{\mathrm{sm}} \subset \mathscr{H}$ такое, что для $\left(f_{1}, \ldots, f_{k}\right) \in U_{\mathrm{sm}}$ полное пересечение $V\left(f_{*}\right)$ есть гладкое подмногообразие коразмерности $k$ в $\mathbb{P}$. Далее, рассмотрим подмножество

$$
Y=\left\{\left(o,\left(f_{*}\right)\right) \mid o \in V\left(f_{*}\right)-\text { нерегулярная точка }\right\}
$$

в $\mathbb{P} \times U_{\mathrm{sm}}$. Мы утверждаем, что замыкание

$$
\overline{\pi(Y)} \subset U_{\mathrm{sm}}
$$

есть собственное замкнутое подмножество положительной коразмерности в $U_{\mathrm{sm}}$. Здесь $\pi: \mathbb{P} \times U_{\mathrm{sm}} \rightarrow U_{\mathrm{sm}}-$ проекция на второй сомножитель. В самом деле, положим

$$
\begin{aligned}
Y(o) & =Y \cap\left(\{o\} \times U_{\mathrm{sm}}\right) \subset \mathbb{P} \times U_{\mathrm{sm}}, \\
I & =\left\{\left(o,\left(f_{*}\right)\right) \mid o \in V\left(f_{*}\right)\right\} \subset \mathbb{P} \times U_{\mathrm{sm}}, \\
I(o) & =I \cap\left(\{o\} \times U_{\mathrm{sm}}\right) \subset \mathbb{P} \times U_{\mathrm{sm}} .
\end{aligned}
$$

Отождествляя $\{o\} \times U_{\mathrm{sm}} \cong U_{\mathrm{sm}}$, можем считать $Y(o)$ и $I(o)$ подмножествами в $U_{\mathrm{sm}}$. 
ЛЕмма 3.9. Выполнена оценка

$$
\operatorname{codim}_{I(o)} Y(o) \geqslant M+k+1 \text {. }
$$

ДокАЗАТЕЛьство. В самом деле, система уравнений $q_{1,1}=\cdots=q_{1, k}=0$ задает касательное пространство $T_{o} V\left(f_{*}\right)$, которое имеет размерность в точности $M$. Проективизируя, получаем набор многочленов

$$
\mathscr{Q}=\left\{q_{i, j} \mid 1 \leqslant i \leqslant k, 2 \leqslant j \leqslant d_{i},(i, j) \neq\left(k, d_{k}\right)\right\}
$$

степени $\geqslant 2$ на $\mathbb{P}^{N}, N=M-1$. Очевидно, что $\sharp \mathscr{Q}=N$. Применяя предложение 3.6 к набору $\mathscr{Q}$ с фиксированными $q_{1,1}, \ldots, q_{1, k}$, видим, что

$$
\begin{aligned}
\operatorname{codim}_{I(o)} Y(o) & \geqslant \min \left\{2 M-1, \sum_{i=1}^{k-1} \sum_{j=2}^{d_{i}}(j-1)+\sum_{j=2}^{d_{k}-1}(j-1)+2\right\} \\
& =\frac{1}{2} \sum_{i=1}^{k-1} d_{i}\left(d_{i}-1\right)+\frac{1}{2}\left(d_{k}-1\right)\left(d_{k}-2\right)+2 .
\end{aligned}
$$

Обозначим последнее выражение через $\xi=\xi\left(d_{1}, \ldots, d_{k}\right)$. Поскольку $M \geqslant 2 k+1$, неравенство (45) вытекает из оценки

$$
\xi\left(d_{1}, \ldots, d_{k}\right) \geqslant M+k+1 .
$$

Вычислим минимум функции $\xi$ вещественных аргументов $\left(a_{1}, \ldots, a_{k}\right) \in \mathbb{R}_{+}^{k}$, удовлетворяющих соотношению $a_{1}+\cdots+a_{k}=M+k$. Очевидно, минимум достигается при $a_{1}=\cdots=a_{k-1}=s \geqslant 0, a_{k}=M+k-(k-1) s \geqslant 0$. Легко подсчитать, что

$$
\begin{aligned}
& \xi(s, \ldots, s, M+k-(k-1) s) \\
& \quad=\frac{1}{2}(k-1)\left(k s^{2}-2(M+k-1) s\right)+\frac{1}{2}(M+k-1)(M+k-2)+2=\zeta(s) .
\end{aligned}
$$

Функция $\zeta(s)$ достигает минимума при $s_{*}=(M+k-1) / k$. Это значение удовлетворяет всем ограничениям. Получаем

$$
\zeta\left(s_{*}\right)=\frac{(M+k-1)(M-1)}{2 k}+2 .
$$

Поскольку $M \geqslant 2 k+1$, получаем $\zeta\left(s_{*}\right) \geqslant M+k+1$, что нам и требуется. Лемма 3.9 доказана.

Поскольку, очевидно, $\operatorname{codim}_{U_{\mathrm{sm}}} I(o)=k$, получаем оценку

$$
\operatorname{codim}_{U_{\mathrm{sm}}} Y(o) \geqslant M+k+(k+1) .
$$

Но множество $\pi(Y)$ покрывается подмножествами $Y(o)$ для всех $o \in \mathbb{P}$, откуда получаем

$$
\operatorname{codim}_{U_{\mathrm{sm}}} \overline{\pi(Y)} \geqslant k+1
$$

так что $\overline{\pi(Y)}$ - собственное подмножество множества $U_{\mathrm{sm}}$. В частности, открытое множество

$$
U_{\text {reg }}=U_{\mathrm{sm}} \backslash \overline{\pi(Y)}
$$

непусто. Предложение 3.5 доказано. 
3.3. Заключительные замечания. В данной главе приведены основные элементы техники гиперкасательных дивизоров. В наиболее трудных задачах (доступных на сегодняшний день) приходится сочетать ее с дополнительными методами (например, с принципом связности Шокурова-Коллара) или применять ее не только к самому многообразию $V$, но и к вспомогательным многообразиям (например, к исключительному дивизору раздутия особой точки $o \in V)$. Дальнейшее усиление техники гиперкасательных дивизоров будет рассмотрено в последующем обзоре, посвященном расслоениям Фано. Здесь отметим лишь, что в последние десять лет предпринималось несколько попыток предложить альтернативную технику, позволяющую исключать бесконечно близкие максимальные особенности на многообразиях Фано произвольной степени, см. [2], [52], [53] (кроме того, имелись препринты, содержавшие ошибки и не дошедшие до журнальных публикаций). Однако метод гиперкасательных дивизоров по эффективности далеко превосходит конструкции этих работ. В частности, ни один из перечисленных альтернативных подходов не позволяет доказать бирациональную сверхжесткость полных пересечений Фано (и тем более не работает в относительном случае, для расслоений Фано). Это обстоятельство, по-видимому, не является случайным. Основой метода гиперкасательных дивизоров является геометрия прямых, лежащих на данном многообразии Фано, - а геометрия рациональных кривых малой (антиканонической) степени имеет исключительно важное значение для бирациональной геометрии самого многообразия.

\section{Список литературы}

[1] В. А. Исковских, "Бирациональная жесткость гиперповерхностей Фано в рамках теории Мори", УМH, 56:2 (2001), 3-86; англ. пер.: V. A. Iskovskikh, "Birational rigidity of Fano hypersurfaces in the framework of Mori theory", Russian Math. Surveys, 56:2 (2001), 207-291.

[2] И. А. Чельцов, "Бирационально жесткие многообразия Фано", УМH, 60:5 (2005), 71-160; англ. пер.: I. A. Cheltsov, "Birationally rigid Fano varieties", Russian Math. Surveys, 60:5 (2005), 875-965.

[3] В.А. Исковских, Ю. И. Манин, “Трехмерные квартики и контрпримеры к проблеме Люрота", Матем. сб., 86:1 (1971), 140-166; англ. пер.: V. A. Iskovskih, J.I. Manin, "Three-dimensional quartics and counterexamples to the Lüroth problem", Sb. Math., 15:1 (1971), 141-166.

[4] Р. Хартсхорн, Алгебраическая геометрия, Мир, М., 1981; пер. с англ.: R. Hartshorne, Algebraic geometry, Grad. Texts in Math., 52, Springer-Verlag, New York-Heidelberg, 1977.

[5] K. Hulek, Elementary algebraic geometry, Stud. Math. Libr., 20, Amer. Math. Soc., Providence, RI, 2003.

[6] Д. Мамфорд, Алгебраическая геометрия. I. Комплексные проективные многообразия, Мир, М., 1979; пер. с англ.: D. Mumford, Algebraic geometry. I. Complex projective varieties, Grundlehren Math. Wiss., 221, Springer-Verlag, Berlin-Heidelberg-New York, 1976.

[7] И. Р. Шафаревич, Основы алгебрачческой геометрии. Т. 1. Алгебраические многообразия в проективном пространстве, 2-е изд., Наука, М., 1988; англ. пер.: I. R. Shafarevich, Basic algebraic geometry. 1: Varieties in projective space, Springer-Verlag, Berlin, 1994. 
[8] H. Clemens, Ph. Griffiths, "The intermediate Jacobian of the cubic threefold", Ann. of Math. (2), 95:2 (1972), 281-356.

[9] V.S. Kulikov, A remark on the non-rationality problem for generic cubic fourfolds, arXiv: math/0610963.

[10] H. Hironaka, "Resolution of singularities of an algebraic variety over a field of characteristic zero. I, II", Ann. of Math. (2), 79:1 (1964), 109-203; 79:2, 205-326.

[11] J. Kollár, Resolution of singularities - Seattle lecture, arXiv: math/0508332v3.

[12] Yu. I. Manin, "Rational surfaces over perfect fields", Inst. Hautes Études Sci. Publ. Math., 30 (1966), 55-113.

[13] Ю.И. Манин, "Рациональные поверхности над совершенными полями. II", Maтем. сб., 72 (1967), 161-192; англ. пер.: Yu. I. Manin, "Rational surfaces over perfect fields. II", Math. USSR-Sb., 1 (1968), 141-168.

[14] M. Noether, "Über Flächen, welche Schaaren rationaler Curven besitzen", Math. Ann., 3:2 (1870), 161-227.

[15] И.Р. Шафаревич (ред.), Алгебраические поверхности, Сборник трудов семинара И. Р. Шафаревича, Тр. МИАН, 75, Наука, М., 1965.

[16] В.А. Исковских, "Бирациональные автоморфизмы трехмерных алгебраических многообразий”, Итоги науки и техники. Соврем. проблемы матем., 12, ВИНИТИ, M., 1979, 159-236.

[17] M. Reid, Birational geometry of 3-folds according to Sarkisov, Preprint, Warwick Univ., 1991.

[18] I. V. Dolgachev, V.A. Iskovskikh, Finite subgroups of the plane Cremona group, arXiv: math/0610595.

[19] H. Hudson, Cremona transformations in plane and space, Cambridge Univ. Press, Cambridge, 1927.

[20] М.Х. Гизатуллин, "Определяющие соотношения для группы Кремоны плоскости”, Изв. АН СССР. Сер. матем., 46:5 (1982), 909-970; англ. пер.: M.H. Gizatullin, "Defining relations for the Cremona group of the plane", Math. USSR-Izv., 21:2 (1983), 211-268.

[21] В.А. Исковских, "Простое доказательство теоремы Гизатуллина", Теория Галуа, кольи, алгебрачческие группы и их приложения, Тр. МИАН, 183, Наука, Л., 1990, 111-116; англ. пер.: V. А. Iskovskikh, "A simple proof of a theorem of Gizatullin", Proc. Steklov Inst. Math., 183 (1991), 127-133.

[22] Ф. Гриффитс, Дж. Харрис, Принципы алгебраической геометрии, Мир, М., 1982; пер. с англ.: Ph. Griffiths, J. Harris, Principles of algebraic geometry, Pure Appl. Math., Wiley, New York, 1978.

[23] G. Fano, "Sopra alcune varieta algebriche a tre dimensioni aventi tutti i generi nulli", Atti Acc. Torino, 43 (1908), 973-977.

[24] G. Fano, "Osservazioni sopra alcune varieta non razionali aventi tutti i generi nulli", Atti Acc. Torino, 50 (1915), 1067-1072.

[25] G. Fano, "Nuove ricerche sulle varieta algebriche a tre dimensioni a curve-sezioni canoniche", Pont. Acad. Sci. Comment., 11 (1947), 635-720.

[26] R. Beheshti, J. M. Starr, Rational surfaces in index-one Fano hypersurfaces, arXiv: math/0601710.

[27] V. A. Iskovskikh, Yu. G. Prokhorov, "Fano varieties", Encyclopaedia Math. Sci., 47, Springer, Berlin, 1999.

[28] C. Birkar, P. Cascini, C. D. Hacon, J. McKernan, Existence of minimal models for varieties of log general type, arXiv: math/0610203.

[29] В.А.Исковских, В.В.Шокуров, “Бирациональные модели и флипы”, УМН, 60:1 (2005), 29-98; англ. пер.: V. A. Iskovskikh, V. V. Shokurov, "Birational models and flips", Russian Math. Surveys, 60:1 (2005), 27-94. 
[30] J. Kollár (ed.), Flips and abundance for algebraic threefolds (Salt Lake City, Utah, 1991), Astérisque, 211, Soc. Math. France, Paris, 1992.

[31] В.В. Шокуров, "Трехмерные логперестройки", Изв. РАН. Сер. матем., 56:1 (1992), 105-203; англ. пер.: V. V. Shokurov, "3-fold log flips", Russian Acad. Sci. Izv. Math., 40:1 (1993), 95-202.

[32] M. Artin, D. Mumford, "Some elementary examples of unirational varieties which are not rational", Proc. London Math. Soc. (3), 25:1 (1972), 75-95.

[33] F. Bardelli, "Polarized mixed Hodge structures: on irrationality of threefolds via degeneration", Ann. Mat. Pura Appl. (4), 137:1 (1984), 287-369.

[34] А.Н. Тюрин, "Средний якобиан трехмерных многообразий", Итоги науки и техники. Соврем. проблемы матем., 12, ВИНИТИ, М., 1979, 1-57.

[35] А. С. Тихомиров, "Промежуточный якобиан двойного $\mathbb{P}^{3}$ с ветвлением в квартике", Изв. АН СССР. Сер. матем., 44:6 (1980), 1329-1377; англ. пер.: А. S. Tihomirov, "The intermediate Jacobian of the double covering of $\mathbb{P}^{3}$ branched at a quartic", Math. USSR-Izv., 17:3 (1981), 523-566.

[36] А. С. Тихомиров, "Особенности тэта-дивизора промежуточного якобиана двойного $\mathbb{P}^{3}$ индекса два", Изв. АН СССР. Сер. матем., 46:5 (1982), 1062-1081; англ. пер.: A.S. Tikhomirov, "Singularities of the theta divisor of the intermediate Jacobian of a double cover of $\mathbb{P}^{3}$ of index two", Math. USSR-Izv., 21:2 (1983), 355-373.

[37] А.С. Тихомиров, "Письмо в редакцию журнала "Известия АН СССР. Серия математическая", Изв. АН СССР. Сер. матем., 49:4 (1985), 891; англ. пер.: A.S. Tikhomirov, "Letter to the editors of the journal "Izvestiya AN SSSR. Seriya Matematicheskaya", Math. USSR-Izv., 27:1 (1986), 201.

[38] В.Г. Саркисов, "Бирациональные автоморфизмы расслоений на коники", Изв. AH CССР. Сер. матем., 44:4 (1980), 918-945; англ. пер.: V. G. Sarkisov, "Birational automorphisms of conic bundles", Math. USSR-Izv., 17:1 (1981), 177-202.

[39] В. Г. Саркисов, "О структурах расслоений на коники", Изв. АН СССР. Сер. матем., 46:2 (1982), 371-408; англ. пер.: V. G. Sarkisov, "On conic bundle structures", Math. USSR-Izv., 20:2 (1983), 355-390.

[40] А.В. Пухликов, "Бирациональные автоморфизмы трехмерных алгебраических многообразий с пучком поверхностей дель Пеццо", Изв. РАН. Сер. матем., 62:1 (1998), 123-164; англ. пер.: A. V. Pukhlikov, "Birational automorphisms of algebraic threefolds with a pencil of del Pezzo surfaces", Izv. Math., 62:1 (1998), 115-155.

[41] Ю. И. Манин, Кубические формы. Алгебра, геометрия, арифметика, Наука, М., 1972; англ. пер.: Yu. I. Manin, Cubic forms. Algebra, geometry, arithmetic, 2nd ed., North-Holland Math. Library, 4, North-Holland, Amsterdam-New York-Oxford, 1986.

[42] В.С. Куликов, "Минимальные объекты алгебраических пространств", Изв. АН СССР. Сер. матем., 50:4 (1986), 726-740; англ. пер.: V.S. Kulikov, "Minimal objects of algebraic spaces", Math. USSR-Izv., 29:1 (1987), 81-94.

[43] F. Campana, "Connexité rationnelle des variétés de Fano", Ann. Sci. École Norm. Sup. (4), 25:5 (1992), 539-545.

[44] J. Kollár, Y. Miyaoka, S. Mori, "Rationally connected varieties", J. Algebraic Geom., 1:3 (1992), 429-448.

[45] J. Kollár, Rational curves on algebraic varieties, Springer-Verlag, Berlin, 1996.

[46] T. Graber, J. Harris, J. Starr, "Families of rationally connected varieties", J. Amer. Math. Soc., 16:1 (2003), 57-67.

[47] B. Hassett, Yu. Tschinkel, "Weak approximation over function fields", Invent. Math., 163:1 (2006), 171-190.

[48] Ф. Кампана, V. Петернелл, А. В. Пухликов, "Обобщенная теорема Тзена и рационально связные расслоения Фано", Матем. сб., 193:10 (2002), 49-74; англ. пер.: F. Campana, T. Peternell, A. V. Pukhlikov, "Generalized Tsen's theorem and rationally connected Fano fibrations", Sb. Math., 193:10 (2002), 1443-1468. 
[49] И.В. Соболев, "Бирациональные автоморфизмы одного класса многообразий, расслоенных на кубические поверхности", Изв. РАН. Сер. матем., 66:1 (2002), 203-224; англ. пер.: I. V. Sobolev, "Birational automorphisms of a class of varieties fibred into cubic surfaces", Izv. Math., 66:1 (2002), 201-222.

[50] A. Corti, "Singularities of linear systems and 3-fold birational geometry", Explicit birational geometry of 3-folds, London Math. Soc. Lecture Note Ser., 281, Cambridge Univ. Press, Cambridge, 2000, 259-312.

[51] A. Corti, M. Mella, "Birational geometry of terminal quartic 3-folds. I", Amer. J. Math., 126:4 (2004), 739-761.

[52] И. А. Чельцов, "О гладкой четырехмерной квинтике”, Матем. сб., 191:9 (2000), 139-160; англ. пер.: I. A. Cheltsov, "On a smooth quintic 4-fold", Sb. Math., 191:9 (2000), 1399-1419.

[53] И. А. Чельцов, "Нерациональность четырехмерного гладкого полного пересечения квадрики и квартики, не содержащего плоскости", Матем. сб., 194:11 (2003), 95-116; англ. пер.: I. A. Cheltsov, "Non-rationality of the 4-dimensional smooth complete intersection of a quadric and a quartic not containing planes", Sb. Math., 194:11 (2003), 1679-1699.

[54] И. А. Чельцов, “Двойное пространство с двойной прямой”, Матем. сб., 195:10 (2004), 109-156; англ. пер.: I. A. Cheltsov, "Double space with double line", Sb. Math., 195:10 (2004), 1503-1544.

[55] И. А. Чельцов, "Расслоения на коники с большим дискриминантом", Изв. РАН. Cер. матем., 68:2 (2004), 215-221; англ. пер.: I. A. Cheltsov, "Conic bundles with big discriminant loci", Izv. Math., 68:2 (2004), 429-434.

[56] I. A. Cheltsov, "Birationally rigid del Pezzo fibrations", Manuscripta Math., 116:4 (2005), 385-396.

[57] I. A. Cheltsov, M. M. Grinenko, Birational rigidity is not an open property, arXiv: math/0612159.

[58] В.А. Исковских, "Факторизация бирациональных отображений рациональных поверхностей с точки зрения теории Мори”, УМH, 51:4 (1996), 3-72; англ. пер.: V.A. Iskovskikh, "Factorization of birational maps of rational surfaces from the viewpoint of Mori theory", Russian Math. Surveys, 51:4 (1996), 585-652.

[59] A. V. Pukhlikov, "Birational isomorphisms of four-dimensional quintics", Invent. Math., 87:2 (1987), 303-329.

[60] А. В. Пухликов, "Бирациональные автоморфизмы двойного пространства и двойной квадрики”, Изв. АН СССР. Сер. матем., 52:1 (1988), 229-239; англ. пер.: A. V. Pukhlikov, "Birational automorphisms of a double space and double quadric", Math. USSR-Izv., 32:1 (1989), 233-243.

[61] А.В. Пухликов, "Бирациональные автоморфизмы трехмерной квартики с простейшей особенностью", Матем. сб., 135:4 (1988), 472-496; англ. пер.: A. V. Pukhlikov, "Birational automorphisms of a three-dimensional quartic with a quadratic singularity", Math. USSR-Sb., 63:2 (1989), 457-482.

[62] А.В. Пухликов, "Замечание о теореме В.А. Исковских и Ю.И. Манина о трехмерной квартике", Теория чисел, алгебра и алгебраическая геометрия, Тр. МИАН, 208, Наука, М., 1995, 278-289; англ. пер.: А. V. Pukhlikov, "A remark on the theorem on a three-dimensional quartic", Proc. Steklov Inst. Math., 208 (1995), 244-254.

[63] A. V. Pukhlikov, "Essentials of the method of maximal singularities", Explicit birational geometry of 3-folds, London Math. Soc. Lecture Note Ser., 281, Cambridge Univ. Press, Cambridge, 2000, 73-100.

[64] A. V. Pukhlikov, "Birational automorphisms of Fano hypersurfaces", Invent. Math., 134:2 (1998), 401-426. 
[65] А. В. Пухликов, "Бирационально жесткие двойные гиперповерхности Фано”, Maтем. сб., 191:6 (2000), 101-126; англ. пер.: A. V. Pukhlikov, "Birationally rigid Fano double hypersurfaces", Sb. Math., 191:6 (2000), 883-908.

[66] А. В. Пухликов, "Бирационально жесткие расслоения Фано", Изв. РАН. Сер. матем., 64:3 (2000), 131-150; англ. пер.: A. V. Pukhlikov, "Birationally rigid Fano fibrations", Izv. Math., 64:3 (2000), 563-581.

[67] A. V. Pukhlikov, "Birationally rigid Fano complete intersections", J. Reine Angew. Math., 541 (2001), 55-79.

[68] А.В. Пухликов, "Бирационально жесткие гиперповерхности Фано с изолированными особенностями", Матем. сб., 193:3 (2002), 135-160; англ. пер.: A. V. Pukhlikov, "Birationally rigid Fano hypersurfaces with isolated singularities", Sb. Math., 193:3 (2002), 445-471.

[69] А. В. Пухликов, "Бирационально жесткие гиперповерхности Фано", Изв. РАН. Cер. матем., 66:6 (2002), 159-186; англ. пер.: A. V. Pukhlikov, "Birationally rigid Fano hypersurfaces", Izv. Math., 66:6 (2002), 1243-1269.

[70] А.В. Пухликов, "Бирационально жесткие итерированные двойные гиперповерхности Фано", Изв. РАН. Сер. матем., 67:3 (2003), 139-182; англ. пер.: A. V. Pukhlikov, "Birationally rigid iterated Fano double covers", Izv. Math., 67:3 (2003), 555-596.

[71] А. В. Пухликов, "Бирационально жесткие многообразия с пучком двойных накрытий Фано. I", Матем. сб., 195:7 (2004), 127-160; англ. пер.: А. V. Pukhlikov, "Birationally rigid varieties with a pencil of double Fano covers. I", Sb. Math., 195:7 (2004), 1039-1071.

[72] А. В. Пухликов, "Бирационально жесткие многообразия с пучком двойных накрытий Фано. II”, Матем. сб., 195:11 (2004), 119-156; англ. пер.: А. V. Pukhlikov, "Birationally rigid varieties with a pencil of Fano double covers. II", Sb. Math., 195:11 (2004), 1665-1702.

[73] A. V. Pukhlikov, Birationally rigid Fano cyclic covers, arXiv: math/0411198.

[74] А.В. Пухликов, "Бирационально жесткие многообразия с пучком двойных накрытий Фано. III", Матем. сб., 197:3 (2006), 35-68; англ. пер.: А. V. Pukhlikov, "Birationally rigid varieties with a pencil of Fano double covers. III", Sb. Math., 197:3 (2006), 335-368.

[75] A. V. Pukhlikov, "Birational geometry of algebraic varieties with a pencil of Fano complete intersections", Manuscripta Math., 121:4 (2006), 491-526.

[76] A. V. Pukhlikov, Birational geometry of algebraic varieties with a pencil of Fano cyclic covers, Preprint series / Max-Planck-Institut fur Mathematik, 2006-66, MPI, Bonn, 2006; http://www.mpim-bonn.mpg.de/preprints/send?bid=2996.

[77] А.В. Пухликов, "Бирациональная геометрия прямых произведений Фано", Изв. РАН. Сер. матем., 69:6 (2005), 153-186; англ. пер.: A. V. Pukhlikov, "Birational geometry of Fano direct products", Izv. Math., 69:6 (2005), 1225-1255.

[78] V.A. Iskovskikh, A. V. Pukhlikov, "Birational automorphisms of multi-dimensional algebraic varieties", J. Math. Sci., 82:4 (1996), 3528-3613.

[79] V. G. Sarkisov, Birational maps of standard $\mathbb{Q}$-Fano fibrations, Preprint, Kurchatov Institute of Atomic Energy, Moscow, 1989.

[80] A. Corti, "Factoring birational maps of threefolds after Sarkisov", J. Algebraic Geom., 4:2 (1995), 223-254.

[81] M. Mella, "Birational geometry of quartic 3-folds. II. The importance of being $\mathbb{Q}$-factorial", Math. Ann., 330:1 (2004), 107-126.

[82] У. Фултон, Теория пересечений, Мир, М., 1989; пер. с англ.: W. Fulton, Intersection theory, Ergeb. Math. Grenzgeb. (3), 2, Springer-Verlag, Berlin, 1984.

[83] A. Corti, A. Pukhlikov, M. Reid, "Fano 3-fold hypersurfaces", Explicit birational geometry of 3-folds, London Math. Soc. Lecture Note Ser., 281, Cambridge Univ. Press, Cambridge, 2000, 175-258. 
[84] М. М. Гриненко, "Бирациональные свойства пучков поверхностей дель Пеццо степеней 1 и 2", Матем. сб., 191:5 (2000), 17-38; англ. пер.: М. M. Grinenko, "Birational properties of pencils of del Pezzo surfaces of degrees 1 and 2", Sb. Math., 191:5 (2000), 633-653.

[85] М.М.Гриненко, "Бирациональные свойства пучков поверхностей дель Пеццо степеней 1 и 2. II", Матем. сб., 194:5 (2003), 31-60; англ. пер.: М. M. Grinenko, "Birational properties of pencils of del Pezzo surfaces of degrees 1 and 2. II", Sb. Math., 194:5 (2003), 669-695.

[86] И.В. Соболев, "Об одной серии бирационально жестких многообразий с пучком гиперповерхностей Фано", Матем. сб., 192:10 (2001), 123-130; англ. пер.: I. V. Sobolev, "On a series of birationally rigid varieties with a pencil of Fano hypersurfaces", Sb. Math., 192:10 (2001), 1543-1551.

А. В. Пухликов (А. V. Pukhlikov)

Математический институт им. В. А. Стеклова РАН

E-mail: pukh@mi.ras.ru
Поступила в редакцию 03.06.2007 